Supporting Information

For

\title{
Spin switching with triazolate-strapped ferrous porphyrins
}

Morten K. Peters, Sebastian Hamer, Torben Jäkel, Fynn Röhrich, Frank D. Sönnichsen, Carolina von Essen, Manu Lahtinen, Christian Näther, Kari Rissanen* and Rainer Herges*

Corresponding Author: **herges@oc.uni-kiel.de

Otto Diels-Institute of Organic Chemistry, Christian-Albrechts-University Kiel, Otto-HahnPlatz 4, 24118 Kiel, Germany 


\section{Table of Contents}

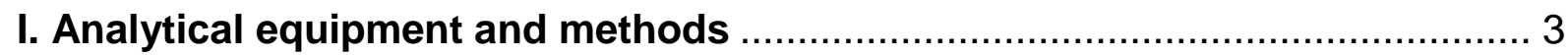

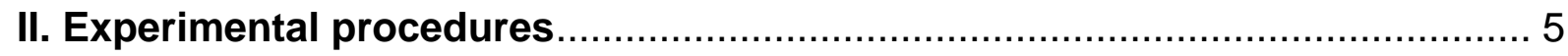

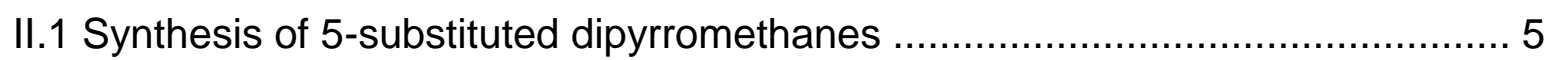

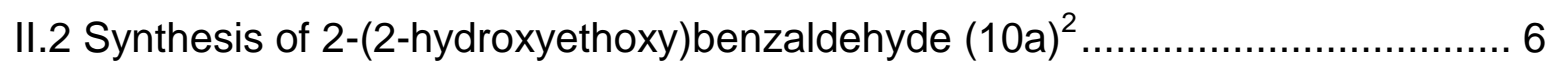

II.3 Synthesis of bis(2-(2-formylphenoxy)ethyl)but-2-ynedioate (13a) ................ 6

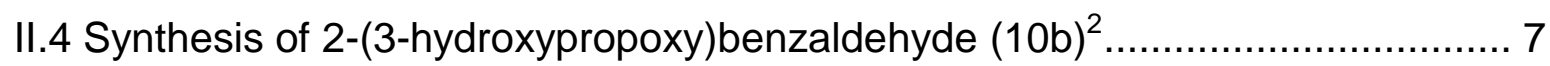

II.5 Synthesis of bis(3-(2-formylphenoxy)propyl)but-2-ynedioate (13b) ............... 8

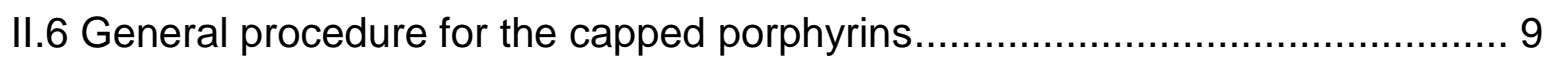

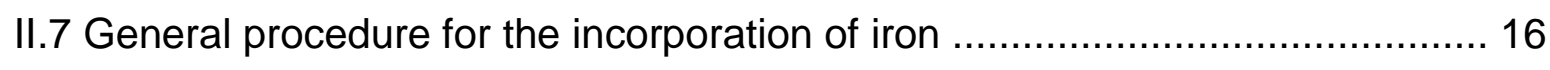

II.8 General procedure for the incorporation of zinc ................................ 19

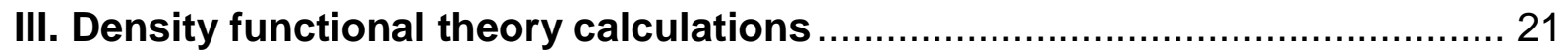

IV. Magnetic Susceptibility - Evans Measurements.................................... 26

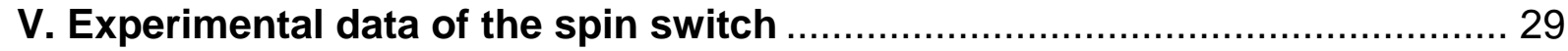

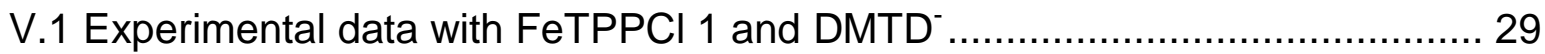

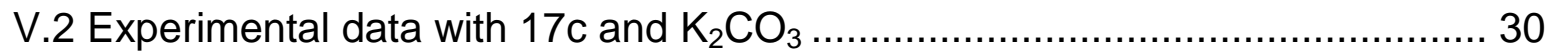

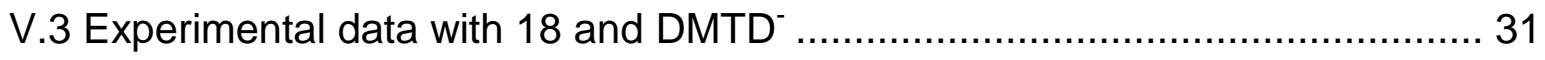

V.4 Experimental data with 18 and $p$-Methoxypyridine ................................ 32

V.5 Experimental data with 18 and 4-chloro-3-(3,5-ditert-butylphenyl)azo-pyridine

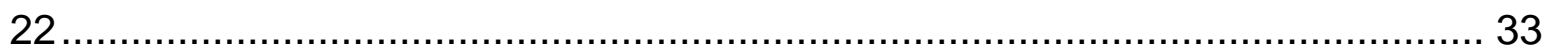

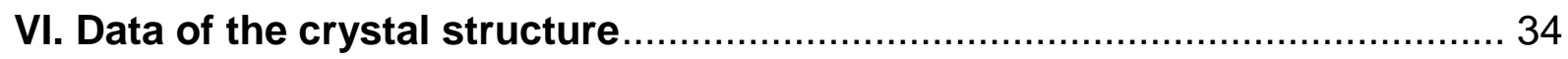

VI.1 Crystal structure of $2^{10}, 2^{20}$-bis(2,6-dichlorophenyl)-4,7,12,15-tetraoxa-2(5,15)porpyhrina-1,3(1,2)-dibenzena-cycloheptadecaphane-9-yn-8,11-dione (15c)...... 34 VII. Photostationary state of trans-4-chloro-3-(3,5-ditert-

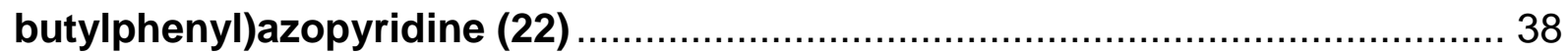

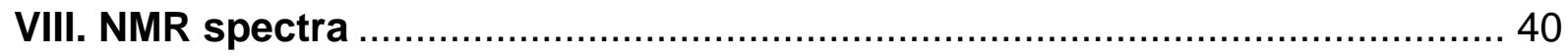




\section{Analytical equipment and methods}

\section{NMR Spectroscopy}

NMR spectra were measured in deuterated solvents (Deutero). The degree of deuteration is given in parentheses. ${ }^{1} \mathrm{H}-\mathrm{NMR}$ spectra are referenced to the following signals:

Acetonitrile- $\mathrm{d}_{3}(99.8 \%): \delta=1.94 \mathrm{ppm}(\mathrm{s})$

Chloroform-d (99.8\%): $\delta=7.26 \mathrm{ppm}(\mathrm{s})$

Dichloromethane- $\mathrm{d}_{2}(99.6 \%): \delta=5.32 \mathrm{ppm}(\mathrm{t})$

DMSO- $d_{6}(99.8 \%): \delta=2.50 \mathrm{ppm}$ (quint)

Toluene $-\mathrm{d}_{8}(99.5 \%): \delta=2.11 \mathrm{ppm}(\mathrm{s})$

The signal multiplicities are abbreviated as follows:

$\mathrm{s}$ : singlet, d: doublet, t: triplet, q: quartet, quint: quintet, m: multiplet, br: broad signal

Measurements were performed by the following instruments:

Bruker DRX 500 and Avance Neo ( ${ }^{1} \mathrm{H}$ NMR: $500 \mathrm{MHz},{ }^{13} \mathrm{C}$ NMR: $\left.125 \mathrm{MHz}\right)$

Bruker AV 600 ( ${ }^{1} \mathrm{H}$ NMR: $600 \mathrm{MHz},{ }^{13} \mathrm{C}$ NMR: $150 \mathrm{MHz}$ )

\section{Mass spectrometry}

The high resolution (HR) mass spectra were measured with an APEX 3 FT-ICR with a 7.05 T magnet by co. Bruker Daltonics. Electron impact (EI). Electrospray ionization (ESI) mass spectra were measured with a Thermo Scientific Q EXACTIVE and Matrix-assisted Laser Desorption/lonization (MALDI) mass spectra were measured with a Biflex III by co. Bruker. Matrix: Cl-CCA 


\section{Chromatography stationary phases}

For column chromatography purifications silica gel (Merck, particle size 0.040-0.063 $\mathrm{mm}$ ) was used. $R_{\mathrm{f}}$ values were determined by thin layer chromatography on Polygram® Sil G/UV254 (Macherey-Nagel, $0.2 \mathrm{~mm}$ particle size).

\section{IR spectroscopy}

Infrared spectra were measured on a Perkin-Elmer 1600 Series FT-IR spectrometer with an A531-G Golden Gate Diamond ATR unit. Signals were abbreviated with w, m, and $s$ for weak, medium and strong intensities. Broad signals were additionally labeled with br.

\section{Elemental analysis}

The amount of carbon, hydrogen and nitrogen in a compound was determined with a CHNSO-Elemental analyser Euro EA 3000 Series by co. Euro Vector. 


\section{Experimental procedures}

\section{II.1 Synthesis of 5-substituted dipyrromethanes}

The different 5 -substituted dipyrromethanes were synthesized as reported. ${ }^{1}$

\section{II.1.1 Synthesis of meso-(pentafluorophenyl)dipyrromethane (14d)}

Yield: $\quad 1.75 \mathrm{~g}(5.61 \mathrm{mmol}, 55 \%)$

${ }^{1} \mathrm{H}-\mathrm{NMR}\left(600 \mathrm{MHz}, 300 \mathrm{~K}, \mathrm{CD}_{2} \mathrm{Cl}_{2}, \mathrm{TMS}\right): \delta=8.22(\mathrm{~s}, \mathrm{br}, 2 \mathrm{H}, \mathrm{N}-\mathrm{H}), 6.70-6.58(\mathrm{~m}$, $2 \mathrm{H}), 6.13-6.11(\mathrm{~m}, 2 \mathrm{H}), 6.00-5.98(\mathrm{~m}, 2 \mathrm{H}), 5.88(\mathrm{~s}, 1 \mathrm{H}) \mathrm{ppm}$.

${ }^{13} \mathrm{C}-\mathrm{NMR}\left(150 \mathrm{MHz}, 300 \mathrm{~K}, \mathrm{CDCl}_{3}\right): \delta=145.1,140.4,138.0,128.5,118.0,116.1$, 108.7, 107.2, $33.1 \mathrm{ppm}$.

${ }^{19} \mathrm{~F}-\mathrm{NMR}\left(470 \mathrm{MHz}, 300 \mathrm{~K}, \mathrm{CDCl}_{3}, \mathrm{CFCl}_{3}\right): \delta=-141.37\left(\mathrm{~d},{ }^{3} \mathrm{~J}=22.4 \mathrm{~Hz}, \mathrm{o}-\mathrm{F}\right),-155.65$ $\left(\mathrm{t},{ }^{3} \mathrm{~J}=20.7 \mathrm{~Hz}, 2 \mathrm{~F}, p-\mathrm{F}\right),-161.03$ up to -16.22 (m, 2F, $\left.m-\mathrm{F}\right) \mathrm{ppm}$.

\section{II.1.2 Synthesis of meso-phenyldipyrromethane (14b)}

Yield: $\quad 1.44 \mathrm{~g}(6.48 \mu \mathrm{mol}, 81 \%)$

${ }^{1} \mathrm{H}-\mathrm{NMR}\left(500 \mathrm{MHz}\right.$ (Bruker DRX 500), $\left.\mathrm{CDCl}_{3}\right): \delta=7.90$ (br s, 2H, N-H), 7.37-7.18 (m, $5 \mathrm{H}), 6.70-6.68(\mathrm{~m}, 2 \mathrm{H}), 6.17-6.15(\mathrm{~m}, 2 \mathrm{H}), 5.93-5.91(\mathrm{~m}, 2 \mathrm{H}), 5.48(\mathrm{~s}, 1 \mathrm{H}) \mathrm{ppm}$.

${ }^{13} \mathrm{C}-\mathrm{NMR}\left(150 \mathrm{MHz}, 300 \mathrm{~K}, \mathrm{CDCl}_{3}\right): \delta=142.2,132.4,128.6,128.5,126.9,117.2$, 108.4, 107.2, $44.1 \mathrm{ppm}$.

\section{II.1.3 Synthesis of dipyrromethane (14a)}

Yield: $\quad 2.37 \mathrm{~g}(16.2 \mathrm{mmol}, 27 \%)$

${ }^{1} \mathrm{H}-\mathrm{NMR}\left(500 \mathrm{MHz}\right.$ (Bruker DRX 500), $\left.300 \mathrm{~K}, \mathrm{CDCl}_{3}\right): \delta=7.87(\mathrm{~s}, \mathrm{br}, 2 \mathrm{H}, \mathrm{NH}$ ), 6.66-664 (m, 2H), 6.17-6.15 (m, 2H), 6.05-6.03 (m, 2H), 3.97 (s, 2H) ppm.

${ }^{13}$ C-NMR $\left(125 \mathrm{MHz}, \mathrm{CDCl}_{3}, 300 \mathrm{~K}\right): \delta=129.2,117.4,108.5,106.5,26.5 \mathrm{ppm}$. 


\section{II.1.4 Synthesis of meso-(dichlorophenyl)dipyrromethane (14c)}

Yield: $1.30 \mathrm{~g}(4.45 \mathrm{mmol}, 39 \%)$

${ }^{1} \mathrm{H}-\mathrm{NMR}\left(500 \mathrm{MHz}\right.$ (Bruker DRX 500), $\mathrm{CDCl}_{3}, 300 \mathrm{~K}$ ): $\delta=8.29$ (s, 2H), 7.33 (d, $\left.{ }^{3} \mathrm{~J}=8.0 \mathrm{~Hz}, 2 \mathrm{H}\right), 7.13\left(\mathrm{t},{ }^{3} \mathrm{~J}=8.0 \mathrm{~Hz}, 1 \mathrm{H}\right), 6.73(\mathrm{~m}, 2 \mathrm{H}), 6.49(\mathrm{~s}, 1 \mathrm{H}), 6.19(\mathrm{~m}, 2 \mathrm{H})$, $6.07(\mathrm{~m}, 2 \mathrm{H}) \mathrm{ppm}$.

${ }^{13} \mathrm{C}$-NMR $\left(125 \mathrm{MHz}, \mathrm{CDCl}_{3}, 300 \mathrm{~K}\right): \delta=137.1,135.9,129.4,128.6,116.9,108.6$, 107.3, 40.0 ppm.

\section{II.2 Synthesis of 2-(2-hydroxyethoxy)benzaldehyde (10a) ${ }^{2}$}

Salicylaldehyde $(9.88 \mathrm{~g}, 80.9 \mathrm{mmol})$ was added dropwise over $15 \mathrm{~min}$ to a stirred solution of sodium hydroxide $(3.20 \mathrm{~g}, 8.10 \mathrm{mmol})$ in water $(60 \mathrm{ml})$. Then 2chloroethanol $(6.36 \mathrm{~g}, 79.0 \mathrm{mmol})$ was added dropwise and the solution was heated at $98^{\circ} \mathrm{C}$ for $16 \mathrm{~h}$. The solution was cooled to room temperature and sodium hydroxide was added until the solution was strongly alkaline $(\mathrm{pH}=10)$. The reaction mixture was extracted four times with dichloromethane $(50 \mathrm{ml})$. The combined organic layers were dried over $\mathrm{MgSO}_{4}$. The crude product was recrystallized from cyclohexane. A colorless solid was obtained.

Yield: $\quad 7.36 \mathrm{~g} \mathrm{(44.4 \textrm {mmol } , 5 6 \% )}$

m.p.: $45^{\circ} \mathrm{C}$

${ }^{1} \mathrm{H}$-NMR (500 MHz (Bruker DRX 500), $300 \mathrm{~K}, \mathrm{CDCl}_{3}$ ): $\delta=10.46(\mathrm{~s}, 1 \mathrm{H}), 7.83$ (dd, ${ }^{3} J=7.7 \mathrm{~Hz},{ }^{4} \mathrm{~J}=1.8 \mathrm{~Hz}, 1 \mathrm{H}$ ), 7.56 (ddd, ${ }^{3} \mathrm{~J}=8.4 \mathrm{~Hz},{ }^{3} \mathrm{~J}=7.7 \mathrm{~Hz},{ }^{4} \mathrm{~J}=1.8 \mathrm{~Hz}, 1 \mathrm{H}$ ), $7.08\left(\mathrm{t},{ }^{3} \mathrm{~J}=7.7 \mathrm{~Hz}, 1 \mathrm{H}\right), 7.01\left(\mathrm{~d},{ }^{3} \mathrm{~J}=8.4 \mathrm{~Hz}, 1 \mathrm{H}\right), 4.23(\mathrm{~m}, 2 \mathrm{H}), 4.03(\mathrm{~m}, 2 \mathrm{H})$, $1.91(\mathrm{~s}, \mathrm{br}, 1 \mathrm{H}, \mathrm{OH}) \mathrm{ppm}$.

${ }^{13}$ C-NMR $(125.1 \mathrm{MHz}, \mathrm{CDCl} 3,300 \mathrm{~K}): \delta=189.9,160.9,136.1,129.8,125.4,121.4$, $113.2,70.4,61.5 \mathrm{ppm}$.

\section{II.3 Synthesis of bis(2-(2-formylphenoxy)ethyl)but-2-ynedioate (13a)}

Acetylenedicarboxylic acid (2.00 g, $17.5 \mathrm{mmol}), 2$-(2-hydroxyethoxy)benzaldehyde (10a) $(1.80 \mathrm{~g}, 14.4 \mathrm{mmol})$ and $p$-toluenesulfonic acid $(150 \mathrm{mg})$ were dissolved under nitrogen atmosphere in benzene $(40 \mathrm{~mL})$ and stirred for $21 \mathrm{~h}$ under reflux. Afterwards the reaction mixture was poured into chloroform $(100 \mathrm{~mL})$ and was washed twice with water. The org. phase was dried over $\mathrm{MgSO}_{4}$ and the solvent was removed under reduced pressure. A yellow oil was obtained.

Yield: $\quad 2.53 \mathrm{~g}(6.18 \mathrm{mmol}, 85 \%)$

${ }^{1} \mathrm{H}-\mathrm{NMR}\left(500 \mathrm{MHz}\right.$ (Bruker DRX 500), $\left.300 \mathrm{~K}, \mathrm{CDCl}_{3}\right): \delta=10.48(\mathrm{~s}, 2 \mathrm{H}), 7.86$ (dd, $\left.{ }^{3} \mathrm{~J}=7.6 \mathrm{~Hz},{ }^{4} \mathrm{~J}=1.7 \mathrm{~Hz}, 2 \mathrm{H}\right), 7.55$ (ddd, ${ }^{3} \mathrm{~J}=8.4 \mathrm{~Hz},{ }^{3} \mathrm{~J}=7.6 \mathrm{~Hz},{ }^{4} \mathrm{~J}=1.7 \mathrm{~Hz}, 2 \mathrm{H}$ ), $7.08\left(\mathrm{t},{ }^{3} \mathrm{~J}=7.6 \mathrm{~Hz}, 2 \mathrm{H}\right) 6.95\left(\mathrm{~d},{ }^{3} \mathrm{~J}=8.4 \mathrm{~Hz}, 2 \mathrm{H}\right), 4.67(\mathrm{~m}, 4 \mathrm{H}), 4.34(\mathrm{~m}, 4 \mathrm{H}) \mathrm{ppm}$. 
${ }^{13} \mathrm{C}-N M R\left(150 \mathrm{MHz}, 300 \mathrm{~K}, \mathrm{CDCl}_{3}\right): \delta=189.5,160.5,151.5,136.0,128.8,125.3$, $121.8,112.5,75.1,66.0,64.6 \mathrm{ppm}$.

MS (MALDI): $\mathrm{m} / \mathrm{z}(\%)=411[\mathrm{M}]^{+}$.

EA: $\left(\mathrm{C}_{22} \mathrm{H}_{18} \mathrm{O}_{8}\right)$

\begin{tabular}{|l|l|l|l|}
\hline & $\mathrm{N} \%$ & $\mathrm{C} \%$ & $\mathrm{H} \%$ \\
\hline calc. & $/$ & 64.39 & 4.42 \\
\hline found & $/$ & 64.99 & 4.40 \\
\hline
\end{tabular}

IR: $\widetilde{v}=2931(\mathrm{w}), 2876(\mathrm{w}), 1719(\mathrm{~s}), 1682(\mathrm{~s}), 1597(\mathrm{~s}), 1484(\mathrm{~m}), 14052(\mathrm{~m})$, $1396(\mathrm{w}), 1372(\mathrm{w}), 1220$ (s), 1161 (s), $1106(\mathrm{~s}), 1040(\mathrm{~s}), 923(\mathrm{~m}), 831(\mathrm{~m}), 751(\mathrm{~s})$, $744(\mathrm{~s}), 653(\mathrm{~m}), 604(\mathrm{w}), 568(\mathrm{w}), 530(\mathrm{w}), 504(\mathrm{w}), 457(\mathrm{w}) \mathrm{cm}^{-1}$.

\section{II.4 Synthesis of 2-(3-hydroxypropoxy)benzaldehyde (10b) ${ }^{2}$}

Salicylaldehyde $(9.00 \mathrm{~g}, 73.8 \mathrm{mmol})$ was added dropwise over $15 \mathrm{~min}$ to a stirred solution of sodium hydroxide $(2.98 \mathrm{~g}, 73.8 \mathrm{mmol})$ in water $(40 \mathrm{ml})$ Then 3chloropropanol $(6.24 \mathrm{~g}, 66.5 \mathrm{mmol})$ was added dropwise and the resulting solution was heated at $98^{\circ} \mathrm{C}$ for $16 \mathrm{~h}$. The solution was cooled to room temperature and sodium hydroxide was added until the solution was strongly alkaline $(\mathrm{pH}=10)$. The reaction mixture was extracted four times with dichloromethane $(50 \mathrm{ml})$. The combined organic layers were dried over $\mathrm{MgSO}_{4}$. The crude product was purified by column chromatography on silica gel (cyclohexane/ethyl acetate 1:2, $R_{f}=0.19$ ). A colorless solid was obtained.

Yield: $\quad 9.27 \mathrm{~g}(51.5 \mathrm{mmol}, 78 \%)$

${ }^{1} \mathrm{H}-\mathrm{NMR}\left(500 \mathrm{MHz}\right.$ (Bruker DRX 500), $300 \mathrm{~K}, \mathrm{CDCl}_{3}$ ): 10.36 (s, $\left.1 \mathrm{H}\right), 7.78$ (dd, ${ }^{3} \mathrm{~J}=7.9 \mathrm{~Hz},{ }^{4} \mathrm{~J}=1.8 \mathrm{~Hz}, 1 \mathrm{H}$ ), 7.53 (ddd, ${ }^{3} \mathrm{~J}=8.4 \mathrm{~Hz},{ }^{3} \mathrm{~J}=7.9 \mathrm{~Hz},{ }^{4} \mathrm{~J}=1.8 \mathrm{~Hz}, 1 \mathrm{H}$ ), $7.03\left(\mathrm{t},{ }^{3} \mathrm{~J}=7.9 \mathrm{~Hz}, 1 \mathrm{H}\right), 7.00\left(\mathrm{~d},{ }^{3} \mathrm{~J}=8.4 \mathrm{~Hz}, 2 \mathrm{H}\right), 4.23\left(\mathrm{t},{ }^{3} \mathrm{~J}=5.9 \mathrm{~Hz}, 2 \mathrm{H}\right), 3.88$ (t, $\left.{ }^{3} J=5.9 \mathrm{~Hz}, 2 \mathrm{H}\right), 2.45(\mathrm{~s}, \mathrm{br}, 1 \mathrm{H}), 2.11\left(\mathrm{p},{ }^{3} \mathrm{~J}=5.9 \mathrm{~Hz}, 2 \mathrm{H}\right) \mathrm{ppm}$.

${ }^{13}$ C-NMR $\left(150 \mathrm{MHz}, 300 \mathrm{~K}, \mathrm{CDCl}_{3}\right): \delta=189.9,161.0,136.0,130.1,124.8,120.7$, $111.8,65.6,60.5,31.9$ ppm. 


\section{II.5 Synthesis of bis(3-(2-formylphenoxy)propyl)but-2-ynedioate (13b)}

Acetylenedicarboxylic acid (627 mg, $4.36 \mathrm{mmol})$, 2-(3-hydroxypropoxy)benzaldehyde $(1.96 \mathrm{~g}, 10.9 \mathrm{mmol})(\mathbf{1 0 b})$ and $p$-toluenesulfonic acid $(50 \mathrm{mg})$ were dissolved under nitrogen atmosphere in benzene $(30 \mathrm{~mL})$ and stirred for $21 \mathrm{~h}$ under reflux. Afterwards the reaction mixture was poured into chloroform $(100 \mathrm{~mL})$ and was washed twice with water. The org. phase was dried over $\mathrm{MgSO}_{4}$ and the solvent was removed under reduced pressure. A yellow solid was obtained.

Yield: $\quad 1.75 \mathrm{~g} \mathrm{(} 4.00 \mathrm{mmol}, 92 \%)$

m.p.: $114^{\circ} \mathrm{C}$

${ }^{1} \mathrm{H}-\mathrm{NMR}$ (500 MHz (Bruker DRX 500), $300 \mathrm{~K}, \mathrm{CDCl}_{3}$ ): 10.41 (s, 2H), 7.84 (dd, $\left.{ }^{3} \mathrm{~J}=7.2 \mathrm{~Hz},{ }^{4} \mathrm{~J}=1.8 \mathrm{~Hz}, 2 \mathrm{H}\right), 7.55$ (ddd, ${ }^{3} \mathrm{~J}=8.4 \mathrm{~Hz},{ }^{3} \mathrm{~J}=7.2 \mathrm{~Hz},{ }^{4} \mathrm{~J}=1.8 \mathrm{~Hz}, 2 \mathrm{H}$ ), $7.05\left(\mathrm{t},{ }^{3} \mathrm{~J}=7.3 \mathrm{~Hz}, 2 \mathrm{H}\right), 6.98\left(\mathrm{~d},{ }^{3} \mathrm{~J}=8.4 \mathrm{~Hz}, 2 \mathrm{H}\right), 4.42\left(\mathrm{t},{ }^{3} \mathrm{~J}=6.1 \mathrm{~Hz}, 4 \mathrm{H}\right), 4.14$ (t, $\left.{ }^{3} J=6.1 \mathrm{~Hz}, 4 \mathrm{H}\right), 2.18\left(\mathrm{p},{ }^{3} J=6.1 \mathrm{~Hz}, 4 \mathrm{H}\right) \mathrm{ppm}$.

${ }^{13}$ C-NMR $\left(150 \mathrm{MHz}, 300 \mathrm{~K}, \mathrm{CDCl}_{3}\right): \delta=189.6,160.8,151.6,136.0,128.7,124.9$, $121.1,112.4,74.8,64.5,63.6,28.2 \mathrm{ppm}$.

MS: (HR) El

\begin{tabular}{|l|l|l|l|}
\hline calc. & found & Mass. Diff $(\mathrm{ppm})$ & Molecular formula \\
\hline 438.13153 & 438.13147 & 0.2 & $\mathrm{C}_{24} \mathrm{H}_{22} \mathrm{FO}_{8}$ \\
\hline
\end{tabular}

IR: $\widetilde{v}=2961(w), 2856(w), 2762(d), 1714(s), 1681(s), 1597(s), 1486(m)$, $1457(\mathrm{~m}), 1385(\mathrm{~m}), 1329(\mathrm{w}), 1289(\mathrm{~m}), 1236(\mathrm{~s}), 1162(\mathrm{~s}), 1129(\mathrm{w}), 1101(\mathrm{~m})$, $1031(\mathrm{~s}), 954(\mathrm{~m}), 927(\mathrm{~m}), 892(\mathrm{w}), 817(\mathrm{~m}), 762(\mathrm{~s}), 744(\mathrm{~s}), 658(\mathrm{~m}), 608(\mathrm{~m})$, $570(\mathrm{~m}), 530(\mathrm{w}) \mathrm{cm}^{-1}$.

EA: $\left(\mathrm{C}_{24} \mathrm{H}_{22} \mathrm{O}_{8}\right)$

\begin{tabular}{|l|l|l|l|}
\hline & $\mathrm{N} \%$ & $\mathrm{C} \%$ & $\mathrm{H} \%$ \\
\hline calc. & $/$ & 65.75 & 5.06 \\
\hline found & $/$ & 65.91 & 5.00 \\
\hline
\end{tabular}




\section{II.6 General procedure for the capped porphyrins}

One equivalent of the dialdehyde and trifluoroboretherate ( 0.1 eq.) were dissolved in dichloromethane $(350 \mathrm{~mL})$ under nitrogen atmosphere. To this mixture, a solution of 5 -substituted dipyrromethane (2 eq.), which was dissolved in dichloromethane $(50 \mathrm{~mL})$, was added under stirring over a period of $1 \mathrm{~h}$. After stirring for $15 \mathrm{~h}, \mathrm{p}$ chloranil (1.5 eq.) was added and stirred for $5 \mathrm{~h}$ at $40^{\circ} \mathrm{C}$. Then the solvent was removed under reduced pressure and the crude product was purified by column chromatography.

II.6.1 Synthesis of $2^{10}, 2^{20}$-bis(2,3,4,5,6-pentafluorophenyl)-4,7,12,15-tetraoxa2(5,15)-porpyhrina-1,3(1,2)-dibenzena-cyclopentadecaphane-9-yn-8,11-dione (15d)

Column chromatography: (dichloromethane/cyclohexane $=3 / 1, R_{\mathrm{f}}=0.18$ )

Yield: $\quad 94.0 \mathrm{mg}(110 \mu \mathrm{mol}, 3 \%)$

m.p.: $\quad 215^{\circ} \mathrm{C}$

${ }^{1} \mathrm{H}-N M R\left(500 \mathrm{MHz}\right.$ (Bruker DRX 500), $\left.300 \mathrm{~K}, \mathrm{CDCl}_{3}\right): \delta=8.86\left(\mathrm{~d},{ }^{3} \mathrm{~J}=4.5 \mathrm{~Hz}, 4 \mathrm{H}\right)$, $8.77\left(\mathrm{~d},{ }^{3} \mathrm{~J}=4.5 \mathrm{~Hz}, 4 \mathrm{H}\right), 8.34\left(\mathrm{dd},{ }^{3} \mathrm{~J}=7.3 \mathrm{~Hz},{ }^{4} \mathrm{~J}=1.6 \mathrm{~Hz}, 2 \mathrm{H}\right), 7.82\left(\mathrm{td},{ }^{3} \mathrm{~J}=8.2 \mathrm{~Hz}\right.$, $\left.{ }^{4} J=1.6 \mathrm{~Hz}, 2 \mathrm{H}\right), 7.56\left(\mathrm{t},{ }^{3} \mathrm{~J}=7.5 \mathrm{~Hz}, 2 \mathrm{H}\right), 7.39\left(\mathrm{~d},{ }^{3} \mathrm{~J}=8.3 \mathrm{~Hz}, 2 \mathrm{H}\right), 3.97-3.91(\mathrm{~m}$, $4 \mathrm{H}), 3.63-3.56(\mathrm{~m}, 4 \mathrm{H}),-2,79(\mathrm{~s}, \mathrm{br}, 2 \mathrm{H}, \mathrm{H}-\mathrm{NH}) \mathrm{ppm}$.

${ }^{19} \mathrm{~F}-\mathrm{NMR}\left(470 \mathrm{MHz}, 300 \mathrm{~K}, \mathrm{CDCl}_{3}, \mathrm{CFCl}_{3}\right): \delta=-135.22\left(\mathrm{dd},{ }^{3} \mathrm{~J}=24.8 \mathrm{~Hz},{ }^{5} \mathrm{~J}=7.0 \mathrm{~Hz}\right.$, $2 \mathrm{~F}, o-\mathrm{F}),-137.06$ (dd, $\left.{ }^{3} \mathrm{~J}=24.8 \mathrm{~Hz},{ }^{5} \mathrm{~J}=7.0 \mathrm{~Hz}, 2 \mathrm{~F}, \mathrm{o}^{\prime}-\mathrm{F}\right),-152.80\left(\mathrm{t},{ }^{3} \mathrm{~J}=20.5 \mathrm{~Hz}, 2 \mathrm{~F}\right.$, $p-\mathrm{F}),-161.99\left(\mathrm{td},{ }^{3} \mathrm{~J}=23.5 \mathrm{~Hz},{ }^{5} \mathrm{~J}=7.0 \mathrm{~Hz}, 2 \mathrm{~F}, m-\mathrm{F}\right),-162.44\left(\mathrm{td},{ }^{3} \mathrm{~J}=23.5 \mathrm{~Hz}\right.$, $\left.{ }^{5} J=7.0 \mathrm{~Hz}, 4 \mathrm{~F}, m^{\prime}-\mathrm{F}\right)$ ppm.

Due to the highly diluted NMR samples, the large number of quaternary C-atoms and the large number of ${ }^{19} \mathrm{~F}$-coupled $\mathrm{C}$-atoms, ${ }^{13} \mathrm{C}$-NMR spectroscopy did not provide sufficient signal intensities. Therefore, the ${ }^{13} \mathrm{C}-\mathrm{NMR}$ spectrum was not analyzable.

MS: (HR) MALDI

\begin{tabular}{|l|l|l|l|}
\hline calc. & found & Mass. Diff (ppm) & Molecular formula \\
\hline 992.16927 & 992.16850 & 0.7 & $\mathrm{C}_{52} \mathrm{H}_{26} \mathrm{~F}_{10} \mathrm{~N}_{4} \mathrm{O}_{6}$ \\
\hline
\end{tabular}

IR: $\widetilde{v}=3322(w), 2926(w), 1723(\mathrm{~m}), 1516(\mathrm{~s}), 1497(\mathrm{~s}), 1447(\mathrm{~m}), 1402(\mathrm{w})$, $1228(\mathrm{~m}), 1112(\mathrm{w}), 1042(\mathrm{~m}), 987(\mathrm{~s}), 921(\mathrm{~s}), 803(\mathrm{~m}), 780(\mathrm{~s}), 757(\mathrm{~s}), 635(\mathrm{w})$, $494(\mathrm{~m}) \mathrm{cm}^{-1}$.

\section{II.6.2 Synthesis of $2^{10}, 2^{20}$-bis(phenyl)-4,7,12,15-tetraoxa-2(5,15)-porpyhrina-} 1,3(1,2)-dibenzena-cyclopentadecaphane-9-yn-8,11-dione (15b)

Column chromatography: (dichloromethane/cyclohexane $=3 / 1, R_{f}=0.25$ )

Yield: $\quad 14.0 \mathrm{mg}(17.2 \mu \mathrm{mol}, 1 \%)$

m.p.: $\quad 230^{\circ} \mathrm{C}$ 
${ }^{1} \mathrm{H}-\mathrm{NMR}\left(500 \mathrm{MHz}\right.$ (Bruker DRX 500), $\left.300 \mathrm{~K}, \mathrm{CDCl}_{3}\right): \delta=8.82\left(\mathrm{~d},{ }^{3} \mathrm{~J}=4.8 \mathrm{~Hz}, 4 \mathrm{H}\right)$, $8.75\left(\mathrm{~d},{ }^{3} \mathrm{~J}=4.8 \mathrm{~Hz}, 4 \mathrm{H}\right), 8.32\left(\mathrm{dd},{ }^{3} J=7.5 \mathrm{~Hz},{ }^{4} \mathrm{~J}=1.7 \mathrm{~Hz}, 2 \mathrm{H}\right), 7.84-7.69(\mathrm{~m}, 12 \mathrm{H})$, $7.56\left(\mathrm{dt},{ }^{3} \mathrm{~J}=7.5 \mathrm{~Hz},{ }^{4} \mathrm{~J}=1.1 \mathrm{~Hz}, 2 \mathrm{H}\right), 7.39\left(\mathrm{dd},{ }^{3} \mathrm{~J}=8.1 \mathrm{~Hz},{ }^{4} \mathrm{~J}=1.1 \mathrm{~Hz} 2 \mathrm{H}\right)$, 3.89-3.90 (m, 4H), 3.63-3.55 (m, 4H), -2,67 (s, br, 2H, H-NH) ppm.

Due to the highly diluted NMR samples, the large number of quaternary $\mathrm{C}$-atoms and poor solubility, ${ }^{13} \mathrm{C}-\mathrm{NMR}$ spectroscopy did not provide sufficient signal intensities. Therefore, the ${ }^{13} \mathrm{C}-\mathrm{NMR}$ spectrum was not analyzable.

MS: (HR) MALDI

\begin{tabular}{|l|l|l|l|}
\hline calc. & found & Mass. Diff (ppm) & Molecular formula \\
\hline 812.26348 & 812.26243 & -1.2 & $\mathrm{C}_{52} \mathrm{H}_{36} \mathrm{~F}_{10} \mathrm{~N}_{4} \mathrm{O}_{6}$ \\
\hline
\end{tabular}

IR: $\widetilde{v}=2925(\mathrm{~m}), 1720(\mathrm{~s}), 1598(\mathrm{~m}), 1446(\mathrm{~m}), 1259(\mathrm{~s}), 1030(\mathrm{~m}), 966(\mathrm{~m}), 801(\mathrm{~s})$, $733(\mathrm{~s}), 701(\mathrm{~s}) \mathrm{cm}^{-1}$.

\section{II.6.3 Synthesis of 4,7,12,15-tetraoxa-2(5,15)-porpyhrina-1,3(1,2)-dibenzena- cyclopentadecaphane-9-yn-8,11-dione (15a)}

Column chromatography: (dichloromethane/n-pentane, $4: 1+1 \%$ methanol, $\left.R_{\mathrm{f}}=0.63\right)$

Yield: $\quad 148 \mathrm{mg}(225 \mu \mathrm{mol}, 8 \%)$

m.p.: $\quad$ decomposition at $256^{\circ} \mathrm{C}$

${ }^{1} \mathrm{H}$-NMR (500 MHz (Bruker DRX 500), $300 \mathrm{~K}, \mathrm{CD}_{2} \mathrm{Cl}_{2}$ ): $\delta=10.30(\mathrm{~s}, 2 \mathrm{H}), 9.39(\mathrm{~d}$, $\left.{ }^{3} J=4.5 \mathrm{~Hz}, 4 \mathrm{H}\right), 8.98\left(\mathrm{~d},{ }^{3} J=4.5 \mathrm{~Hz}, 4 \mathrm{H}\right), 8.38\left(\mathrm{dd},{ }^{3} J=7.4 \mathrm{~Hz},{ }^{4} J=1.6 \mathrm{~Hz}, 2 \mathrm{H}\right)$, 7.87 (ddd, ${ }^{3} \mathrm{~J}=8.4 \mathrm{~Hz},{ }^{3} \mathrm{~J}=7.4 \mathrm{~Hz},{ }^{4} \mathrm{~J}=1.6 \mathrm{~Hz}, 2 \mathrm{H}$ ), 7.61 (ddd, ${ }^{3} \mathrm{~J}=8.4 \mathrm{~Hz}$, $\left.{ }^{3} \mathrm{~J}=7.4 \mathrm{~Hz},{ }^{4} \mathrm{~J}=1.2 \mathrm{~Hz}, 2 \mathrm{H}\right), 7.47\left(\mathrm{dd},{ }^{3} \mathrm{~J}=8.4 \mathrm{~Hz},{ }^{4} \mathrm{~J}=1.2 \mathrm{~Hz}, 2 \mathrm{H}\right), 3.94-3.90(\mathrm{~m}$, $4 \mathrm{H}), 3.51-3.47(\mathrm{~m}, 4 \mathrm{H}),-3.15(\mathrm{~s}, b r, 2 \mathrm{H}, \mathrm{NH}) \mathrm{ppm}$.

Due to the highly diluted NMR samples, the large number of quaternary $\mathrm{C}$-atoms and poor solubility, ${ }^{13} \mathrm{C}$-NMR spectroscopy did not provide sufficient signal intensities. Therefore, the ${ }^{13} \mathrm{C}-\mathrm{NMR}$ spectrum was not analyzable.

MS: (HR) MALDI

\begin{tabular}{|l|l|l|l|}
\hline calc. & found & Mass. Diff (ppm) & Molecular formula \\
\hline 660.20088 & 660.19943 & -2.1 & $\mathrm{C}_{40} \mathrm{H}_{28} \mathrm{~N}_{4} \mathrm{O}_{6}$ \\
\hline
\end{tabular}

IR: $\widetilde{v}=3296(w), 2924(w), 1713(s), 1573(w), 1482(m), 1451(m), 1412(m)$, $1237(\mathrm{~s}), 1207(\mathrm{~s}), 1162(\mathrm{~m}), 1143(\mathrm{~m}), 1108(\mathrm{~m}), 1055(\mathrm{~m}), 1036(\mathrm{~m}), 973(\mathrm{~m})$, $957(\mathrm{~s}), 921(\mathrm{~m}), 849(\mathrm{~s}) \mathrm{cm}^{-1}$ 
EA: $\left(\mathrm{C}_{40} \mathrm{H}_{28} \mathrm{~N}_{4} \mathrm{O}_{6}\right)$

\begin{tabular}{|l|l|l|l|}
\hline & $\mathrm{N} \%$ & $\mathrm{C} \%$ & $\mathrm{H} \%$ \\
\hline calc. & 8.48 & 72.72 & 4.27 \\
\hline found & 8.12 & 71.92 & 4.11 \\
\hline
\end{tabular}

II.6.4 Synthesis of $2^{10}, 2^{20}$-bis(2,6-dichlorophenyl)-4,7,12,15-tetraoxa-2(5,15)porpyhrina-1,3(1,2)-dibenzena-cycloheptadecaphane-9-yn-8,11-dione (15c)

Column chromatography: (dichloromethane $R_{\mathrm{f}}=0.57$ )

Yield: $\quad 136 \mathrm{mg}(143 \mathrm{mmol}, 15 \%)$

m.p.: $\quad$ decomposition at $28{ }^{\circ} \mathrm{C}$

${ }^{1} \mathrm{H}-\mathrm{NMR}\left(500 \mathrm{MHz}\right.$ (Bruker DRX 500), $\left.\mathrm{CDCl}_{3}, 300 \mathrm{~K}\right): \delta=8.77\left(\mathrm{~d},{ }^{3} \mathrm{~J}=4.6 \mathrm{~Hz}, 4 \mathrm{H}\right.$ ), $8.63\left(\mathrm{~d},{ }^{3} \mathrm{~J}=4.6 \mathrm{~Hz}, 4 \mathrm{H}\right), 8.34$ (dd, ${ }^{3} J=7.4 \mathrm{~Hz},{ }^{4} J=1.6 \mathrm{~Hz}, 2 \mathrm{H}$ ), 7.84 (dd, $\left.{ }^{3} J=8.1 \mathrm{~Hz},{ }^{4} \mathrm{~J}=1.4 \mathrm{~Hz}, 2 \mathrm{H}\right), 7.76\left(\mathrm{td},{ }^{3} \mathrm{~J}=8.2 \mathrm{~Hz},{ }^{4} \mathrm{~J}=1.6 \mathrm{~Hz}, 2 \mathrm{H}\right), 7.71$ (dd, $\left.{ }^{3} \mathrm{~J}=8.1 \mathrm{~Hz},{ }^{4} \mathrm{~J}=1.4 \mathrm{~Hz}, 2 \mathrm{H}^{\prime}\right), 7.66\left(\mathrm{t},{ }^{3} \mathrm{~J}=8.1 \mathrm{~Hz}, 2 \mathrm{H}\right), 7.51 \quad\left(\mathrm{td},{ }^{3} \mathrm{~J}=7.4 \mathrm{~Hz}\right.$, $\left.{ }^{4} J=1.0 \mathrm{~Hz}, 2 \mathrm{H}\right), 7.34\left(\mathrm{dd},{ }^{3} \mathrm{~J}=8.2 \mathrm{~Hz},{ }^{4} J=1.0 \mathrm{~Hz}, 2 \mathrm{H}\right), 3.92(\mathrm{~m}, 4 \mathrm{H}), 3.59(\mathrm{~m}, 4 \mathrm{H})$, $-2.46(\mathrm{~s}, \mathrm{br}, 2 \mathrm{H}, \mathrm{NH}) \mathrm{ppm}$.

Due to the highly diluted NMR samples, the large number of quaternary $\mathrm{C}$-atoms, ${ }^{13} \mathrm{C}$-NMR spectroscopy did not provide sufficient signal intensities. Therefore, the ${ }^{13} \mathrm{C}-\mathrm{NMR}$ spectrum was not analyzable.

MS: (HR) MALDI

\begin{tabular}{|l|l|l|l|}
\hline calc. & found & Mass. Diff (ppm) & Molecular formula \\
\hline 948.10759 & 948.10630 & -1.3 & $\mathrm{C}_{52} \mathrm{H}_{32} \mathrm{Cl}_{4} \mathrm{~N}_{4} \mathrm{O}_{6}$ \\
\hline
\end{tabular}

IR: $\widetilde{v}=3312(w), 2922(w), 2250(w), 1723(m), 1557(m), 1488(m), 1427(m)$, $1344(\mathrm{~m}), 1222(\mathrm{~s}), 1114(\mathrm{~m}), 1065(\mathrm{~s}), 1045(\mathrm{~s}), 965(\mathrm{~s}), 925(\mathrm{~m}), 800(\mathrm{~s}), 730(\mathrm{~s})$, $710(\mathrm{~s}), 649(\mathrm{~m}), 543(\mathrm{w}) \mathrm{cm}^{-1}$.

II.6.5 Synthesis of $2^{10}, 2^{20}$-bis(2,3,4,5,6-pentafluorophenyl)-4,8,13,17-tetraoxa2(5,15)-porpyhrina-1,3(1,2)-dibenzena-cycloheptadecaphane-10-yn-9,12-dione (15f)

Column chromatography: (dichloromethane $R_{\mathrm{f}}=0.87$ )

Yield: $\quad 89.8 \mathrm{mg}(88.0 \mu \mathrm{mol}, 5 \%)$

m.p.: $\quad 202^{\circ} \mathrm{C}$

${ }^{1} \mathrm{H}-\mathrm{NMR}\left(500 \mathrm{MHz}\right.$ (Bruker DRX 500), $\left.300 \mathrm{~K}, \mathrm{CDCl}_{3}\right): \delta=8.90\left(\mathrm{~d},{ }^{3} \mathrm{~J}=4.6 \mathrm{~Hz}, 4 \mathrm{H}\right)$, $8.76\left(\mathrm{~d},{ }^{3} \mathrm{~J}=4.6 \mathrm{~Hz}, 4 \mathrm{H}\right), 8.07$ (dd, ${ }^{3} \mathrm{~J}=7.5 \mathrm{~Hz},{ }^{4} \mathrm{~J}=1.7 \mathrm{~Hz}, 2 \mathrm{H}$ ), 7.79 (ddd, $\left.{ }^{3} \mathrm{~J}=8.3 \mathrm{~Hz},{ }^{3} \mathrm{~J}=7.5 \mathrm{~Hz},{ }^{4} \mathrm{~J}=1.7 \mathrm{~Hz}, 2 \mathrm{H}\right), 7.45\left(\mathrm{td},{ }^{3} \mathrm{~J}=7.5 \mathrm{~Hz},{ }^{4} \mathrm{~J}=1.0 \mathrm{~Hz}, 2 \mathrm{H}\right), 7.37$ 
(dd, $\left.{ }^{3} J=8.3 \mathrm{~Hz},{ }^{4} \mathrm{~J}=1.0 \mathrm{~Hz}, 2 \mathrm{H}\right), 3.80\left(\mathrm{t},{ }^{3} \mathrm{~J}=5.6 \mathrm{~Hz}, 4 \mathrm{H}\right), 3.30\left(\mathrm{t},{ }^{3} \mathrm{~J}=6.1 \mathrm{~Hz}, 4 \mathrm{H}\right.$ ), 1.47-1.41 (m, $4 \mathrm{H}),-2,71$ (s, br, 2H, H-NH) ppm.

Due to the highly diluted NMR samples, the large number of quaternary $\mathrm{C}$-atoms and the large number of ${ }^{19} \mathrm{~F}$-coupled $\mathrm{C}$-atoms, ${ }^{13} \mathrm{C}$-NMR spectroscopy did not provide sufficient signal intensities. Therefore, the ${ }^{13} \mathrm{C}-\mathrm{NMR}$ spectrum was not analyzable.

${ }^{19} \mathrm{~F}$-NMR $\left(470 \mathrm{MHz}, 300 \mathrm{~K}, \mathrm{CDCl}_{3}, \mathrm{CFCl}_{3}\right): \delta=-135.83\left(\mathrm{dd},{ }^{3} \mathrm{~J}=23.9 \mathrm{~Hz},{ }^{5} \mathrm{~J}=7.4 \mathrm{~Hz}\right.$, $2 \mathrm{~F}, o-\mathrm{F}),-136.90\left(\mathrm{dd},{ }^{3} \mathrm{~J}=23.9 \mathrm{~Hz},{ }^{5} \mathrm{~J}=8.1 \mathrm{~Hz}, 2 \mathrm{~F}, \mathrm{o}^{\prime}-\mathrm{F}\right),-152.74\left(\mathrm{t},{ }^{3} \mathrm{~J}=20.9 \mathrm{~Hz}, 2 \mathrm{~F}\right.$, $p-\mathrm{F}$ ), -161.97 (td, ${ }^{3} \mathrm{~J}=22.3 \mathrm{~Hz},{ }^{5} \mathrm{~J}=7.4 \mathrm{~Hz}, 2 \mathrm{~F}, m-\mathrm{F}$ ), -162.40 (td, ${ }^{3} \mathrm{~J}=22.3 \mathrm{~Hz}$, $\left.{ }^{5} J=8.1 \mathrm{~Hz}, 4 \mathrm{~F}, m^{\prime}-\mathrm{F}\right) \mathrm{ppm}$.

MS: (HR) EI

\begin{tabular}{|l|c|c|c|}
\hline calc. & found & Mass. Diff $(\mathrm{ppm})$ & Molecular formula \\
\hline 1020.20057 & 1020.19808 & -2.49 & $\mathrm{C}_{54} \mathrm{H}_{30} \mathrm{~F}_{10} \mathrm{~N}_{4} \mathrm{O}_{6}$ \\
\hline
\end{tabular}

IR: $\widetilde{v}=3372(\mathrm{~m}), 1722(\mathrm{~m}), \quad 1516(\mathrm{~m}), 1495(\mathrm{~s}), 1409(\mathrm{~s}), 1307(\mathrm{~s}), 1200(\mathrm{~s})$, $1112(\mathrm{~m}), 1042(\mathrm{~m}), 987(\mathrm{~s}), 921(\mathrm{~s}), 883(\mathrm{~s}), 802(\mathrm{~m}), 777(\mathrm{~m}), 709(\mathrm{~s}), 502(\mathrm{~m})$, $462(\mathrm{~s}), 434(\mathrm{~s}), 425(\mathrm{~m}), 404(\mathrm{~m}) \mathrm{cm}^{-1}$.

II.6.6 Synthesis of $2^{10}, 2^{20}$-bis(2,6-dichlorophenyl)-4,8,13,17-tetraoxa-2(5,15)porpyhrina-1,3(1,2)-dibenzena-cyclopentadecaphane-10-yn-9,12-dione (15e)

Column chromatography: (dichloromethane $R_{\mathrm{f}}=0.55$ )

Yield: $\quad 1.08 \mathrm{~g}(1.08 \mathrm{mmol}, 35 \%)$

m.p..: decomposition at $260^{\circ} \mathrm{C}$

${ }^{1} \mathrm{H}-\mathrm{NMR}\left(500 \mathrm{MHz}\right.$ (Bruker DRX 500), $\left.300 \mathrm{~K}, \mathrm{CDCl}_{3}\right): \delta=8.82\left(\mathrm{~d},{ }^{3} \mathrm{~J}=4.7 \mathrm{~Hz}, 4 \mathrm{H}\right)$, $8.64\left(\mathrm{~d},{ }^{3} \mathrm{~J}=4.7 \mathrm{~Hz}, 4 \mathrm{H}\right), 8.28\left(\mathrm{dd},{ }^{3} \mathrm{~J}=7.4 \mathrm{~Hz},{ }^{4} \mathrm{~J}=1.7 \mathrm{~Hz}, 2 \mathrm{H}\right), 7.84$ (dd, $\left.{ }^{3} \mathrm{~J}=8.1 \mathrm{~Hz},{ }^{4} \mathrm{~J}=1.3 \mathrm{~Hz}, 2 \mathrm{H}\right), 7.77-7.72(\mathrm{~m}, 4 \mathrm{H}), 7.70\left(\mathrm{t},{ }^{3} \mathrm{~J}=8.1 \mathrm{~Hz}, 2 \mathrm{H}\right), 7.46$ (td, $\left.{ }^{3} \mathrm{~J}=7.4 \mathrm{~Hz},{ }^{4} \mathrm{~J}=1.0 \mathrm{~Hz}, 2 \mathrm{H}\right), 7.26\left(\mathrm{dd},{ }^{3} \mathrm{~J}=8.2 \mathrm{~Hz},{ }^{4} \mathrm{~J}=1.0 \mathrm{~Hz}, 2 \mathrm{H}\right), 3.73(\mathrm{t}$, $\left.{ }^{3} J=5.8 \mathrm{~Hz}, 4 \mathrm{H}\right), 3.45\left(\mathrm{t},{ }^{3} \mathrm{~J}=6.8 \mathrm{~Hz}, 4 \mathrm{H}\right), 1.44-1.35(\mathrm{~m}, 4 \mathrm{H}),-2,45(\mathrm{~s}, \mathrm{br}, 2 \mathrm{H}$, $\mathrm{H}-\mathrm{NH}) \mathrm{ppm}$.

Due to the highly diluted NMR samples, the large number of quaternary C-atoms, ${ }^{13} \mathrm{C}$-NMR spectroscopy did not provide sufficient signal intensities. Therefore, the ${ }^{13} \mathrm{C}-\mathrm{NMR}$ spectrum was not analyzable.

MS: (HR) MALDI

\begin{tabular}{|l|c|c|c|}
\hline calc. & found & Mass. Diff (ppm) & Molecular formula \\
\hline 976.13889 & 976.13750 & -1.4 & $\mathrm{C}_{54} \mathrm{H}_{36} \mathrm{Cl}_{4} \mathrm{~N}_{4} \mathrm{O}_{6}$ \\
\hline
\end{tabular}

IR: $\widetilde{v}=3321(\mathrm{w}), 1722(\mathrm{~s}), 1556(\mathrm{~s}), 1426(\mathrm{~s}), 1345(\mathrm{w}), 1231(\mathrm{~s}), 1115(\mathrm{w}), 1044(\mathrm{~m})$, 964 (s), 800 (s), 750 (s), $712(\mathrm{~s}), 640(\mathrm{~m}), 565(\mathrm{w}) \mathrm{cm}^{-1}$. 


\section{II.7 General procedure for the capped porphyrin with triazole}

The capped porphyrin (1 eq.) and trimethylsilyl azide (100 eq.) are dissolved in toluene $(10 \mathrm{~mL})$ and the mixture was stirred at $80^{\circ} \mathrm{C}$ for $18 \mathrm{~h}$. Then the solvent was removed under reduced pressure and the crude product was taken up in dichloromethane $(50 \mathrm{~mL})$ and was washed twice with water $(30 \mathrm{~mL})$. Then the solvent was removed under reduced pressure and the crude product was purified by column chromatography.

\section{II.7.1 Synthesis of $2^{10}, 2^{20}$-bis(2,3,4,5,6-pentafluorophenyl)-4,7,11,14-tetraoxa-} 2(5,15)-porpyhrina-9(4,5)-1,2,3-triazola-1,3(1,2)-dibenzena-cyclotetradecaphane8,10-dione (16d)

Column chromatography: (dichloromethane/methanol, 97:3, $R_{f}=0.48$ )

Yield: $\quad 7.00 \mathrm{mg}(6.76 \mu \mathrm{mol}, 81 \%)$

${ }^{1} \mathrm{H}-\mathrm{NMR}\left(500 \mathrm{MHz}\right.$ (Bruker DRX 500), $300 \mathrm{~K}$, Aceton- $\left.\mathrm{d}_{6}\right): \delta=9.02(\mathrm{~s}, \mathrm{br}, 4 \mathrm{H}), 8.87$ (s, $\mathrm{br}, 4 \mathrm{H}), 8.44\left(\mathrm{dd},{ }^{3} \mathrm{~J}=7.3 \mathrm{~Hz},{ }^{4} \mathrm{~J}=1.7 \mathrm{~Hz}, 2 \mathrm{H}\right), 7.82\left(\mathrm{td},{ }^{3} \mathrm{~J}=8.0 \mathrm{~Hz},{ }^{4} \mathrm{~J}=1.7 \mathrm{~Hz}, 2 \mathrm{H}\right)$, $7.56\left(\mathrm{t},{ }^{3} \mathrm{~J}=7.5 \mathrm{~Hz}, 2 \mathrm{H}\right), 7.39\left(\mathrm{~d},{ }^{3} \mathrm{~J}=8.2 \mathrm{~Hz}, 2 \mathrm{H}\right), 4.10-4.04(\mathrm{~m}, 4 \mathrm{H}), 3.52-3.46(\mathrm{~m}$, $4 \mathrm{H}),-2,93$ (s, br, $2 \mathrm{H}, \mathrm{H}-\mathrm{NH}) \mathrm{ppm}$.

Due to the highly diluted NMR samples, the large number of quaternary $\mathrm{C}$-atoms and the large number of ${ }^{19} \mathrm{~F}$-coupled $\mathrm{C}$-atoms, ${ }^{13} \mathrm{C}$-NMR spectroscopy did not provide sufficient signal intensities. Therefore, the ${ }^{13} \mathrm{C}$-NMR spectrum was not analyzable.

${ }^{19} \mathrm{~F}$-NMR $\left(470 \mathrm{MHz}, 300 \mathrm{~K}\right.$, Aceton- $\left.\mathrm{d}_{6}, \mathrm{CFCl}_{3}\right): \delta=-140.32\left(\mathrm{dd},{ }^{3} \mathrm{~J}=23.7 \mathrm{~Hz}\right.$, $\left.{ }^{4} J=8.4 \mathrm{~Hz}, 4 \mathrm{~F}, o-\mathrm{F}\right),-156.49\left(\mathrm{t},{ }^{3} \mathrm{~J}=20.5 \mathrm{~Hz}, 2 \mathrm{~F}, p-\mathrm{F}\right),-164.65$ up to $-165.48(\mathrm{~m}, 4 \mathrm{~F}$, $m-\mathrm{F})$ ppm.

MS: (HR) MALDI

\begin{tabular}{|l|l|l|l|}
\hline calc. & found & Mass. Diff (ppm) & Molecular formula \\
\hline 1035.18631 & 1035.18833 & 1.9 & $\mathrm{C}_{52} \mathrm{H}_{27} \mathrm{~F}_{10} \mathrm{~N}_{7} \mathrm{O}_{6}$ \\
\hline
\end{tabular}

IR: $\widetilde{v}=2923(\mathrm{~m}), 2855(\mathrm{~m}), 1733(\mathrm{~m}), 1516(\mathrm{~m}), 1496(\mathrm{~s}), 1445(\mathrm{~m}), 1245(\mathrm{~m})$, $1082(\mathrm{~s}), 985$ (s), 920 (s), 801 (s), 778 (s), $756(\mathrm{~s}), 721(\mathrm{~s}), 657(\mathrm{~m}), 633(\mathrm{~m}), 530(\mathrm{~s})$, $493(\mathrm{~s}) \mathrm{cm}^{-1}$.

II.7.2 Synthesis of $2^{10}, 2^{20}$-bis(phenyl)-4,7,11,14-tetraoxa-2(5,15)-porpyhrina9(4,5)-1,2,3-triazola-1,3(1,2)-dibenzena-cyclotetradecaphane-8,10-dione (16b)

Column chromatography: (dichloromethane/methanol, 97:3, $R_{\mathrm{f}}=0.33$ )

Yield: $\quad 7.00 \mathrm{~g}(8.19 \mu \mathrm{mol}, 52 \%)$

Due to the low yield of the starting material, the product was further reacted directly.

MS (MALDI, TOF): $m / z(\%)=856[\mathrm{M}]^{+}$ 


\section{II.7.3 Synthesis of 4,7,11,14-tetraoxa-2(5,15)-porpyhrina-9(4,5)-1,2,3-triazola- 1,3(1,2)-dibenzena-cyclotetradecaphane-8,10-dione (16a)}

Acetonitrile was used as solvent.

Column chromatography: (dichloromethane/methanol, 98:2, $R_{\mathrm{f}}=0.16$ )

Yield: $\quad 49.5 \mathrm{mg}(70.3 \mu \mathrm{mol}, 34 \%)$

m.p.: $\quad$ decomposition at $245^{\circ} \mathrm{C}$

${ }^{1}$ H-NMR $(500 \mathrm{MHz}$ (Bruker DRX 500), DMSO-d $6,300 \mathrm{~K}$ ): $\delta=10.12(\mathrm{~s}, 2 \mathrm{H}), 9.41$ (d, $\left.{ }^{3} J=4.6 \mathrm{~Hz}, 4 \mathrm{H}\right), 8.86\left(\mathrm{~d},{ }^{3} \mathrm{~J}=4.6 \mathrm{~Hz}, 4 \mathrm{H}\right), 8.56\left(\mathrm{dd},{ }^{3} \mathrm{~J}=7.2 \mathrm{~Hz},{ }^{4} J=1.7 \mathrm{~Hz}, 2 \mathrm{H}\right)$, $7.87\left(\mathrm{td},{ }^{3} \mathrm{~J}=8.1 \mathrm{~Hz},{ }^{4} \mathrm{~J}=1.7 \mathrm{~Hz}, 2 \mathrm{H}\right), 7.61\left(\mathrm{td},{ }^{3} \mathrm{~J}=7.4 \mathrm{~Hz},{ }^{4} \mathrm{~J}=0.8 \mathrm{~Hz}, 2 \mathrm{H}\right), 7.42$ (dd, $\left.{ }^{3} \mathrm{~J}=8.4 \mathrm{~Hz},{ }^{4} \mathrm{~J}=0.8 \mathrm{~Hz}, 2 \mathrm{H}\right), 4.03-3.96(\mathrm{~m}, 4 \mathrm{H}), 3.29-3.19(\mathrm{~m}, 4 \mathrm{H}),-3.41(\mathrm{~s}, 2 \mathrm{H}$, $\mathrm{NH}) \mathrm{ppm}$;

Due to the highly diluted NMR samples, the large number of quaternary $\mathrm{C}$-atoms and poor solubility, ${ }^{13} \mathrm{C}-\mathrm{NMR}$ spectroscopy did not provide sufficient signal intensities. Therefore, the ${ }^{13} \mathrm{C}-\mathrm{NMR}$ spectrum was not analyzable.

MS (MALDI): $\mathrm{m} / \mathrm{z}(\%)=704[\mathrm{M}]^{+}$.

MS: (HR) ESI

\begin{tabular}{|l|l|l|l|}
\hline calc. & found & Mass. Diff (ppm) & Molecular formula \\
\hline 704.22521 & 704.22469 & $-0,73$ & $\mathrm{C}_{40} \mathrm{H}_{30} \mathrm{~F}_{10} \mathrm{~N}_{7} \mathrm{O}_{6}$ \\
\hline
\end{tabular}

IR: $\widetilde{v}=2925(\mathrm{w}), 2852(\mathrm{w}), 1728(\mathrm{~s}), 1579(\mathrm{~m}), 1488(\mathrm{~m}), 1444(\mathrm{~m}), 1370(\mathrm{~m})$, $1299(\mathrm{~m}), 1255(\mathrm{~s}), 1144(\mathrm{~m}), 1116(\mathrm{~s}), 1069(\mathrm{~s}), 972(\mathrm{~m}), 957(\mathrm{~s}), 926(\mathrm{~m}), 855(\mathrm{~s})$, $788(\mathrm{~s}), 753(\mathrm{~s}), 690(\mathrm{~m}) \mathrm{cm}^{-1}$.

II.7.4 Synthesis of $2^{10}, 2^{20}$-bis(2,6-dichlorophenyl)-4,7,11,14-tetraoxa-2(5,15)porpyhrina-9(4,5)-1,2,3-triazola-1,3(1,2)-dibenzena-cyclotetradecaphane-8,10dione (16c)

Column chromatography: (dichlormethane/methanol, 97:3, $R_{\mathrm{f}}=0.57$ )

Yield: $\quad 10.0 \mathrm{mg}(10.1 \mathrm{mmol}, 48 \%)$

m.p.: $\quad$ decomposition at $270^{\circ} \mathrm{C}$

${ }^{1} \mathrm{H}-\mathrm{NMR}\left(500 \mathrm{MHz}\right.$ (Bruker DRX 500), $\left.\mathrm{CD}_{2} \mathrm{Cl}_{2}, 300 \mathrm{~K}\right): \delta=8.84\left(\mathrm{~d},{ }^{3} \mathrm{~J}=4.6 \mathrm{~Hz}, 4 \mathrm{H}\right)$, $8.65\left(\mathrm{~d},{ }^{3} \mathrm{~J}=4.6 \mathrm{~Hz}, 4 \mathrm{H}\right), 8.34$ (dd, ${ }^{3} \mathrm{~J}=7.5 \mathrm{~Hz},{ }^{4} \mathrm{~J}=1.5 \mathrm{~Hz}, 2 \mathrm{H}$ ), 7.93 (dd, $\left.{ }^{3} \mathrm{~J}=6.5 \mathrm{~Hz},{ }^{4} \mathrm{~J}=3.1 \mathrm{~Hz}, 2 \mathrm{H}\right), 7.81\left(\mathrm{td},{ }^{3} \mathrm{~J}=8.2 \mathrm{~Hz},{ }^{4} \mathrm{~J}=1.5 \mathrm{~Hz}, 2 \mathrm{H}\right), 7.78-7.75(\mathrm{~m}$, $4 \mathrm{H}), 7.52\left(\mathrm{td},{ }^{3} \mathrm{~J}=7.5 \mathrm{~Hz},{ }^{4} \mathrm{~J}=0.7 \mathrm{~Hz}, 2 \mathrm{H}\right), 7.31\left(\mathrm{~d},{ }^{3} \mathrm{~J}=8.2 \mathrm{~Hz},{ }^{4} \mathrm{~J}=0.7 \mathrm{~Hz}, 2 \mathrm{H}\right.$ ), $4.07(\mathrm{~m}, 4 \mathrm{H}), 3.61(\mathrm{~m}, 4 \mathrm{H}),-3.20(\mathrm{~s}, \mathrm{br}, 2 \mathrm{H}, \mathrm{NH}) \mathrm{ppm}$.

Due to the highly diluted NMR samples, the large number of quaternary C-atoms, ${ }^{13} \mathrm{C}$-NMR spectroscopy did not provide sufficient signal intensities. Therefore, the ${ }^{13} \mathrm{C}-\mathrm{NMR}$ spectrum was not analyzable. 
MS: (HR) MALDI

\begin{tabular}{|l|l|l|l|}
\hline calc. & found & Mass. Diff $(\mathrm{ppm})$ & Molecular formula \\
\hline 991.12464 & 991.12609 & 3.4 & $\mathrm{C}_{52} \mathrm{H}_{33} \mathrm{Cl}_{4} \mathrm{~N}_{7} \mathrm{O}_{6}$ \\
\hline
\end{tabular}

EA: $\left(\mathrm{C}_{52} \mathrm{H}_{33} \mathrm{~N}_{7} \mathrm{O}_{6} \mathrm{Cl}_{4}\right)$

\begin{tabular}{|l|l|l|l|}
\hline & $\mathrm{N} \%$ & $\mathrm{C} \%$ & $\mathrm{H} \%$ \\
\hline calc. & 9.87 & 62.85 & 3.35 \\
\hline found & 9.22 & 61.51 & 3.54 \\
\hline
\end{tabular}

IR: $\widetilde{v}=2921(w), 1735(m), 1646(m), 1556(w), 1426(m), 1344(m), 1290(m)$, $1114(\mathrm{~m}), 1083(\mathrm{~m}), 980(\mathrm{~m}), 800(\mathrm{~s}), 753(\mathrm{~s}), 712(\mathrm{~m}), 651(\mathrm{~s}) \mathrm{cm}^{-1}$.

\section{II.7.5 Synthesis of $2^{10}, 2^{20}$-bis(2,3,4,5,6-pentafluorophenyl)-4,8,12,16-tetraoxa- 2(5,15)-porpyhrina-10(4,5)-1,2,3-triazola-1,3(1,2)-dibenzena- cyclohexadecaphane-9,11-dione (16f)}

Column chromatography: (dichloromethane/methanol, 97:3, $R_{\mathrm{f}}=0.37$ )

Yield: $\quad 31.3 \mathrm{mg}(29.4 \mu \mathrm{mol}, 60 \%)$

m.p.: $\quad$ decomposition at $179^{\circ} \mathrm{C}$

${ }^{1} \mathrm{H}-\mathrm{NMR}\left(500 \mathrm{MHz}\right.$ (Bruker DRX 500), $\left.300 \mathrm{~K}, \mathrm{CD}_{2} \mathrm{Cl}_{2}\right): \delta=8.93\left(\mathrm{~d},{ }^{3} \mathrm{~J}=4.5 \mathrm{~Hz}, 4 \mathrm{H}\right)$, $8.83\left(\mathrm{~d},{ }^{3} \mathrm{~J}=4.5 \mathrm{~Hz}, 4 \mathrm{H}\right), 8.21$ (dd, ${ }^{3} \mathrm{~J}=7.4 \mathrm{~Hz},{ }^{4} \mathrm{~J}=1.7 \mathrm{~Hz}, 2 \mathrm{H}$ ), 7.83 (ddd, $\left.{ }^{3} J=8.3 \mathrm{~Hz},{ }^{3} \mathrm{~J}=7.4 \mathrm{~Hz},{ }^{4} \mathrm{~J}=1.7 \mathrm{~Hz}, 2 \mathrm{H}\right), 7.48\left(\mathrm{td},{ }^{3} \mathrm{~J}=7.4 \mathrm{~Hz},{ }^{4} \mathrm{~J}=1.0 \mathrm{~Hz}, 2 \mathrm{H}\right), 7.35$ (dd, $\left.{ }^{3} \mathrm{~J}=8.3 \mathrm{~Hz},{ }^{4} \mathrm{~J}=1.0 \mathrm{~Hz}, 2 \mathrm{H}\right), 4.01\left(\mathrm{t},{ }^{3} \mathrm{~J}=5.4 \mathrm{~Hz}, 4 \mathrm{H}\right), 2.71\left(\mathrm{t},{ }^{3} \mathrm{~J}=8.3 \mathrm{~Hz}, 4 \mathrm{H}\right)$, 1.39-1.33 (m, $4 \mathrm{H}),-2,84(\mathrm{~s}, \mathrm{br}, 2 \mathrm{H}, \mathrm{H}-\mathrm{NH}) \mathrm{ppm}$.

Due to the highly diluted NMR samples, the large number of quaternary $\mathrm{C}$-atoms and the large number of ${ }^{19} \mathrm{~F}$-coupled $\mathrm{C}$-atoms, ${ }^{13} \mathrm{C}$-NMR spectroscopy did not provide sufficient signal intensities. Therefore, the ${ }^{13} \mathrm{C}$-NMR spectrum was not analyzable.

${ }^{19} \mathrm{~F}$-NMR $\left(470 \mathrm{MHz}, \quad 300 \mathrm{~K}, \mathrm{CD}_{2} \mathrm{Cl}_{2}, \mathrm{CFCl}_{3}\right): \delta=-136.85$ (dd, ${ }^{3} \mathrm{~J}=23.9 \mathrm{~Hz}$, $\left.{ }^{5} \mathrm{~J}=7.9 \mathrm{~Hz}, 2 \mathrm{~F}, o-\mathrm{F}\right),-137.96\left(\mathrm{dd},{ }^{3} \mathrm{~J}=23.9 \mathrm{~Hz},{ }^{5} \mathrm{~J}=7.9 \mathrm{~Hz}, 2 \mathrm{~F}, o^{\prime}-\mathrm{F}\right),-153.93$ (t, $\left.{ }^{3} J=20.7 \mathrm{~Hz}, 2 \mathrm{~F}, p-\mathrm{F}\right),-163.03\left(\mathrm{td},{ }^{3} \mathrm{~J}=22.4 \mathrm{~Hz},{ }^{5} \mathrm{~J}=7.9 \mathrm{~Hz}, 2 \mathrm{~F}, m-\mathrm{F}\right),-163.28(\mathrm{td}$, $\left.{ }^{3} J=22.4 \mathrm{~Hz},{ }^{5} \mathrm{~J}=7.9 \mathrm{~Hz}, 4 \mathrm{~F}, m^{\prime}-\mathrm{F}\right) \mathrm{ppm}$.

MS: (HR) EI

\begin{tabular}{|l|c|c|c|}
\hline calc. & found & Mass. Diff $(\mathrm{ppm})$ & Molecular formula \\
\hline 1063.21761 & 1063.21846 & 0.79 & $\mathrm{C}_{54} \mathrm{H}_{31} \mathrm{~F}_{10} \mathrm{~N}_{7} \mathrm{O}_{6}$ \\
\hline
\end{tabular}

IR: $\widetilde{v}=2925(\mathrm{w}), 1738(\mathrm{~m}), 1516(\mathrm{~s}), 1495(\mathrm{~s}), 1446(\mathrm{~m}), 1345(\mathrm{w}), 1287(\mathrm{~m})$, $1193(\mathrm{~m}), 1089(\mathrm{~m}), 1042(\mathrm{~m}), 972(\mathrm{~s}), 920(\mathrm{~s}), 829(\mathrm{~m}), 755(\mathrm{~s}), 724(\mathrm{~m}), 657(\mathrm{~m})$, $637(w), 528(w), 463(w), 428(w) \mathrm{cm}^{-1}$. 
II.7.6 Synthesis of $2^{10}, 2^{20}$-bis(2,6-dichlorophenyl)-4,8,12,16-tetraoxa-2(5,15)porpyhrina-10(4,5)-1,2,3-triazola-1,3(1,2)-dibenzena-cyclohexadecaphane-9,11dione (16e)

Column chromatography: (dichloromethane/methanol, 97:3, $R_{\mathrm{f}}=0.10$ )

Yield: $\quad 114 \mathrm{mg}(111 \mu \mathrm{mol}, 96 \%)$

m.p.: $\quad$ decomposition at $272{ }^{\circ} \mathrm{C}$

${ }^{1} \mathrm{H}-\mathrm{NMR}\left(500 \mathrm{MHz}\right.$ (Bruker DRX 500), $\left.300 \mathrm{~K}, \mathrm{CD}_{2} \mathrm{Cl}_{2}\right): \delta=8.83\left(\mathrm{~d},{ }^{3} \mathrm{~J}=4.8 \mathrm{~Hz}, 4 \mathrm{H}\right)$, $8.65\left(\mathrm{~d},{ }^{3} \mathrm{~J}=4.8 \mathrm{~Hz}, 4 \mathrm{H}\right), 8.30\left(\mathrm{dd},{ }^{3} \mathrm{~J}=7.3 \mathrm{~Hz},{ }^{4} \mathrm{~J}=1.7 \mathrm{~Hz}, 2 \mathrm{H}\right), 7.83-7.77(\mathrm{~m}, 4 \mathrm{H})$, $7.77\left(\mathrm{dd},{ }^{3} \mathrm{~J}=8.3 \mathrm{~Hz},{ }^{4} \mathrm{~J}=1.4 \mathrm{~Hz}, 2 \mathrm{H}\right), 7.71\left(\mathrm{t},{ }^{3} \mathrm{~J}=8.1 \mathrm{~Hz}, 2 \mathrm{H}\right), 7.47\left(\mathrm{td},{ }^{3} \mathrm{~J}=7.6 \mathrm{~Hz}\right.$, ${ }^{4} J=0.8 \mathrm{~Hz}, 2 \mathrm{H}$ ), 7.29 (dd, $\left.{ }^{3} J=8.4 \mathrm{~Hz},{ }^{4} J=0.8 \mathrm{~Hz}, 2 \mathrm{H}\right), 3.96\left(\mathrm{t},{ }^{3} J=5.5 \mathrm{~Hz}, 4 \mathrm{H}\right.$ ), $2.77\left(\mathrm{t},{ }^{3} \mathrm{~J}=8.0 \mathrm{~Hz}, 4 \mathrm{H}\right), 1.40-1.19(\mathrm{~m}, 4 \mathrm{H}),-2,62(\mathrm{~s}, \mathrm{br}, 2 \mathrm{H}, \mathrm{H}-\mathrm{NH}) \mathrm{ppm}$.

Due to the highly diluted NMR samples, the large number of quaternary C-atoms, ${ }^{13} \mathrm{C}$-NMR spectroscopy did not provide sufficient signal intensities. Therefore, the ${ }^{13} \mathrm{C}-\mathrm{NMR}$ spectrum was not analyzable.

MS: (HR) MALDI

\begin{tabular}{|l|c|c|c|}
\hline calc. & found & Mass. Diff $(\mathrm{ppm})$ & Molecular formula \\
\hline 1019.15594 & 1019.15333 & -2.5 & $\mathrm{C}_{54} \mathrm{H}_{37} \mathrm{Cl}_{4} \mathrm{~N}_{7} \mathrm{O}_{6}$ \\
\hline
\end{tabular}

IR: $\widetilde{v}=2922(\mathrm{w}), 1732(\mathrm{~m}), 1557(\mathrm{~m}), 1427(\mathrm{~m}), 1345(\mathrm{~m}), 1288(\mathrm{~m}), 1186(\mathrm{~m})$, $1088(\mathrm{~m}), 1051(\mathrm{~s}), 980(\mathrm{~m}), 965(\mathrm{~s}), 879(\mathrm{~m}), 800(\mathrm{~s}), 753(\mathrm{~m}), 730(\mathrm{w}), 712(\mathrm{~m})$, $653(\mathrm{~m}), 564(\mathrm{~m}) \mathrm{cm}^{-1}$.

\section{II.7 General procedure for the incorporation of iron}

The crapped porphyrin (1 eq.) and iron(II)chloride tetrahydrate (18 eq.) were dissolved in degassed acetonitrile $(30 \mathrm{~mL})$ under nitrogen atmosphere and refluxed for $4 \mathrm{~h}$. Then the solvent was removed under reduced pressure and the crude product was solved dichloromethane $(40 \mathrm{~mL})$, washed twice with brine $(50 \mathrm{~mL})$ and water $(100 \mathrm{~mL})$. The combined organic layers were dried over magnesium sulfate and the solvent was removed under reduced pressure.

II.8.1 Synthesis of $2^{10}, 2^{20}$-bis(2,3,4,5,6-pentafluorophenyl)-4,7,11,14-tetraoxa2(5,15)-iron(III)porpyhrina-9(4,5)-1,2,3-triazola-1,3(1,2)-dibenzenacyclotetradecaphane-8,10-dione chloride (17e)

Column chromatography: (dichloromethane/methanol $=94 / 6, R_{f}=0.24$ )

Yield: $\quad 1.38 \mathrm{mg}(1.23 \mu \mathrm{mol}, 32 \%)$

${ }^{1} \mathrm{H}-\mathrm{NMR}\left(600 \mathrm{MHz}, \mathrm{CD}_{2} \mathrm{Cl}_{2}, 300 \mathrm{~K}\right.$ ): $\delta=87.19$ (s, br, 2H, Pyrrol-H), 83.45 (s, br, 2H, Pyrrol-H), 80.78 (s, br, 2H, Pyrrol-H), 79.40 (s, br, 2H, Pyrrol-H) ppm.

Due to the paramagnetic, the NMR-spectroscopy was impossible. 
MS: (HR) MALDI

\begin{tabular}{|l|l|l|l|}
\hline calc. & found & Mass. Diff $(\mathrm{ppm})$ & Molecular formula \\
\hline 1089.10561 & 1089.10387 & 1.5 & $\mathrm{C}_{52} \mathrm{H}_{25} \mathrm{~F}_{10} \mathrm{FeN}_{7} \mathrm{O}_{6}$ \\
\hline
\end{tabular}

IR: $\widetilde{v}=3318$ (w), 2924 (s), 2854 (s), 1446 (s), $1717(\mathrm{~s}), 1599(\mathrm{w}), 1519(\mathrm{~m}), 1497$ (s), $1445(\mathrm{~m}), 1375(\mathrm{~m}), 1288(\mathrm{~m}), 1116(\mathrm{~m}), 1087(\mathrm{~m}), 1051(\mathrm{~m}), 988(\mathrm{~s}), 938(\mathrm{~m})$, $921(\mathrm{~m}), 843(\mathrm{~m}), 802(\mathrm{~m}), 781(\mathrm{~m}), 760(\mathrm{~s}), 672(\mathrm{w}) \mathrm{cm}^{-1}$.

II.8.2 Synthesis of $\quad 2^{10}, 2^{20}$-bis(phenyl)-4,7,11,14-tetraoxa-2(5,15)iron(III)porpyhrina-9(4,5)-1,2,3-triazola-1,3(1,2)-dibenzena-cyclotetradecaphane8,10-dione chloride (17b)

Column chromatography: (dichloromethane/methanol $=94 / 6, R_{\mathrm{f}}=0.51$ )

Yield: $\quad 4.64 \mathrm{mg}(4.91 \mu \mathrm{mol}, 60 \%)$

${ }^{1} \mathrm{H}-\mathrm{NMR}\left(600 \mathrm{MHz}, \mathrm{CD}_{2} \mathrm{Cl}_{2}, 300 \mathrm{~K}\right): \delta=77.82$ (s, br, 8H, Pyrrol-H) ppm.

Due to the paramagnetism, no conclusive NMR spectrum could be obtained.

MS: (HR) MALDI

\begin{tabular}{|l|l|l|l|}
\hline calc. & found & Mass. Diff $(\mathrm{ppm})$ & Molecular formula \\
\hline 909.19995 & 909.19964 & 0.3 & $\mathrm{C}_{52} \mathrm{H}_{35} \mathrm{FeN}_{7} \mathrm{O}_{6}$ \\
\hline
\end{tabular}

IR: $\widetilde{v}=3363$ (br. w), 2924 (s), 2854 (s), 1737 (s), 1455 (m), 1376 (m), 1202 (m), $1073(\mathrm{~m}), 1061(\mathrm{~m}), 1000(\mathrm{~m}), 885(\mathrm{w}), 755(\mathrm{~m}), 719(\mathrm{~m}) \mathrm{cm}^{-1}$.

\section{II.8.3 Synthesis of 4,7,11,14-tetraoxa-2(5,15)-iron(III)porpyhrina-9(4,5)-1,2,3-} triazola-1,3(1,2)-dibenzena-cyclotetradecaphane-8,10-dione chloride (17a)

Column chromatography (Alumina silica gel): (dichloromethane/methanol =94/6, $\left.R_{\mathrm{f}}=0.09\right)$

Yield: $\quad 10.0 \mathrm{mg}(12.6 \mu \mathrm{mol}, 12 \%)$

${ }^{1} \mathrm{H}-\mathrm{NMR}\left(500 \mathrm{MHz}\left(\right.\right.$ Bruker DRX 500), $\left.\mathrm{CD}_{2} \mathrm{Cl}_{2}, 300 \mathrm{~K}\right): \delta=80.49$ (s, br, 4H), 74.86 (s, br, 4H) ppm.

Due to the paramagnetism, no conclusive NMR spectrum could be obtained.

MS: (HR) MALDI

\begin{tabular}{|l|l|l|l|}
\hline calc. & found & Mass. Diff (ppm) & Molecular formula \\
\hline 792.10619 & 792.1106 & 5.5 & $\mathrm{C}_{40} \mathrm{H}_{27} \mathrm{FeCIN}_{4} \mathrm{O}_{6}$ \\
\hline
\end{tabular}

IR: $\widetilde{v}=3358(w), 2922(m), 2852(m), 1736(w), 1606(w), 1632(w), 1491(m)$, $1447(\mathrm{~m}), 1379(\mathrm{w}), 1292(\mathrm{w}), 1258(\mathrm{w}), 1064(\mathrm{~m}), 998(\mathrm{~m}), 957(\mathrm{~s}), 930(\mathrm{w}), 884(\mathrm{w})$, $791(\mathrm{w}), 749(\mathrm{~m}), 720(\mathrm{~m}), 700(\mathrm{~m}), 631(\mathrm{~s}), 538(\mathrm{~s}) \mathrm{cm}^{-1}$. 
II.8.4 Synthesis of $2^{10}, 2^{20}$-bis(2,6-dichlorophenyl)-4,7,11,14-tetraoxa-2(5,15)iron(III)porpyhrina-9(4,5)-1,2,3-triazola-1,3(1,2)-dibenzena-cyclotetradecaphane8,10-dione chloride (17c)

Column chromatography: (dichloromethane/methanol $=94 / 6, R_{\mathrm{f}}=0.90$ )

Yield: $\quad 4.50 \mathrm{mg}(4.30 \mu \mathrm{mol}, 42 \%)$

m.p.: $\quad$ decomposition at $175^{\circ} \mathrm{C}$

${ }^{1} \mathrm{H}-\mathrm{NMR}\left(500 \mathrm{MHz}\right.$ (Avance Neo), Acetonitrile-d $\mathrm{d}_{3}, 300 \mathrm{~K}$ ): $\delta=85.87$ (s, br, $2 \mathrm{H}$, H-Pyrrol), 82.91 (s, br, 2H, H-Pyrrol), 80.29 (s, br, 2H, H-Pyrrol), 78.44 (s, br, 2H, H-Pyrrol) ppm.

Due to the paramagnetism, no conclusive NMR spectrum could be obtained.

MS: (HR) MALDI

\begin{tabular}{|l|l|l|l|}
\hline calc. & found & Mass. Diff (ppm) & Molecular formula \\
\hline 1045.04418 & 1045.04704 & 2.6 & $\mathrm{C}_{52} \mathrm{H}_{31} \mathrm{Cl}_{4} \mathrm{FeN}_{7} \mathrm{O}_{6}$ \\
\hline
\end{tabular}

IR: $\widetilde{v}=2922(w), 2853(w), 2347(w), 2251(m), 1733(m), 1427(m), 1258(m)$, $1202(\mathrm{~m}), 1067$ (s), 998 (s), 931 (w), 837 (w), 801 (s), $755(\mathrm{~m}), 716(\mathrm{~m}), 653(\mathrm{w})$, $565(w) \mathrm{cm}^{-1}$.

II.8.5 Synthesis of $2^{10}, 2^{20}$-bis(2,6-dichlorophenyl)-4,8,12,16-tetraoxa-2(5,15)iron(III)porpyhrina-10(4,5)-1,2,3-triazola-1,3(1,2)-dibenzena-

cyclohexadecaphane-9,11-dione chloride (17d)

Without column chromatography.

Yield: $\quad 18.8 \mathrm{mg}(17.0 \mu \mathrm{mol}, 69 \%)$

${ }^{1} \mathrm{H}-\mathrm{NMR}\left(500 \mathrm{MHz}\right.$ (Bruker DRX 500), $\mathrm{CD}_{2} \mathrm{Cl}_{2}, 300 \mathrm{~K}$ ): $\delta=83.58$ (s, br, 2H, H-Pyrrol), 80.41 (s, br, 2H, H-Pyrrol), 78.94 (s, br, 2H, H-Pyrrol), 77.34 (s, br, 2H, H-Pyrrol) ppm.

Due to the paramagnetism, no conclusive NMR spectrum could be obtained.

MS: (HR) MALDI

\begin{tabular}{|l|c|c|c|}
\hline calc. & found & Mass. Diff (ppm) & Molecular formula \\
\hline 1108.04317 & 1108.04317 & -1.0 & $\mathrm{C}_{54} \mathrm{H}_{35} \mathrm{Cl}_{5} \mathrm{FeN}_{7} \mathrm{O}_{6}$ \\
\hline
\end{tabular}

IR: $\widetilde{v}=2923(\mathrm{w}), 1732(\mathrm{~m}), 1557(\mathrm{~m}), 1427(\mathrm{~m}), 1328(\mathrm{~m}), 1203(\mathrm{~m}), 1055(\mathrm{~m})$, $996(\mathrm{~s}), 837(\mathrm{w}), 802(\mathrm{~s}), 754(\mathrm{~m}), 716(\mathrm{~s}), 654(\mathrm{~m}), 550(\mathrm{~m}), 408(\mathrm{~s}) \mathrm{cm}^{-1}$. 


\section{II.8 General procedure for the incorporation of zinc}

The capped porphyrin (1 eq.) and zinc acetylacetonate tetrahydrate (18 eq.) were dissolved in acetonitrile $(30 \mathrm{~mL})$ and refluxed for $4 \mathrm{~h}$. Then the solvent was removed under reduced pressure and the crude product was solved in dichloromethane $(40 \mathrm{~mL})$, washed twice with brine $(50 \mathrm{~mL})$ and water $(100 \mathrm{~mL})$. The combined organic layers were dried over magnesium sulfate and the solvent was removed under reduced pressure.

II.8.1 Synthesis of $2^{10}, 2^{20}$-bis(2,6-dichlorophenyl)-4,7,11,14-tetraoxa-2(5,15)zinc(II)porpyhrina-9(4,5)-1,2,3-triazola-1,3(1,2)-dibenzena-cyclotetradecaphane8,10-dione

Column chromatography: (dichloromethane/methanol $=97 / 3, R_{\mathrm{f}}=0.28$ )

Yield: $\quad 15.9 \mathrm{mg}(15.1 \mu \mathrm{mol}, 100 \%)$

m.p.: $\quad 127^{\circ} \mathrm{C}$

${ }^{1} \mathrm{H}-\mathrm{NMR}\left(500 \mathrm{MHz}\left(\right.\right.$ Bruker DRX 500), $\left.\mathrm{CD}_{2} \mathrm{Cl}_{2}, 300 \mathrm{~K}\right): \delta=8.89\left(\mathrm{~d},{ }^{3} \mathrm{~J}=4.5 \mathrm{~Hz}, 4 \mathrm{H}\right)$, $8.69\left(\mathrm{~d},{ }^{3} \mathrm{~J}=4.5 \mathrm{~Hz}, 4 \mathrm{H}\right), 8.45\left(\mathrm{dd},{ }^{3} \mathrm{~J}=7.4 \mathrm{~Hz},{ }^{4} \mathrm{~J}=1.5 \mathrm{~Hz}, 2 \mathrm{H}\right), 7.87$ (dd, $\left.{ }^{3} \mathrm{~J}=8.1 \mathrm{~Hz},{ }^{4} \mathrm{~J}=1.6 \mathrm{~Hz}, 2 \mathrm{H}\right), 7.79\left(\mathrm{td},{ }^{3} \mathrm{~J}=8.2 \mathrm{~Hz},{ }^{4} \mathrm{~J}=1.5 \mathrm{~Hz}, 2 \mathrm{H}\right.$ ), 7.78 (dd, $\left.{ }^{3} \mathrm{~J}=8.1 \mathrm{~Hz},{ }^{4} \mathrm{~J}=1.6 \mathrm{~Hz}, 2 \mathrm{H}\right), 7.78-7.75\left(\mathrm{t},{ }^{3} \mathrm{~J}=8.1 \mathrm{~Hz}, 2 \mathrm{H}\right), 7.54\left(\mathrm{td},{ }^{3} \mathrm{~J}=7.4 \mathrm{~Hz}\right.$, $\left.{ }^{4} \mathrm{~J}=0.7 \mathrm{~Hz}, 2 \mathrm{H}\right), 7.31\left(\mathrm{~d},{ }^{3} \mathrm{~J}=8.2 \mathrm{~Hz}, 2 \mathrm{H}\right), 3.97(\mathrm{~s}, \mathrm{br}, 4 \mathrm{H}), 3.34(\mathrm{~s}, \mathrm{br}, 4 \mathrm{H}) \mathrm{ppm}$.

MS (MALDI, TOF): $m / z(\%)=1054[\mathrm{M}]^{+}$.

MS: (HR) MALDI

\begin{tabular}{|l|c|c|c|}
\hline calc. & found & Mass. Diff $(\mathrm{ppm})$ & Molecular formula \\
\hline 1053.03814 & 1053.03928 & 1.1 & $\mathrm{C}_{52} \mathrm{H}_{31} \mathrm{Cl}_{4} \mathrm{~N}_{7} \mathrm{ZnO}_{6}$ \\
\hline
\end{tabular}

IR: $\widetilde{v}=2922(\mathrm{~s}), 2853(\mathrm{~s}), 2362(\mathrm{w}), 1737(\mathrm{~s}), 1556(\mathrm{w}), 1487(\mathrm{w}), 1443(\mathrm{~m}), 1426(\mathrm{~s})$, $1377(\mathrm{~m}), 1336(\mathrm{w}), 1245(\mathrm{~s}), 1203(\mathrm{~m}), 1118(\mathrm{~m}), 1064(\mathrm{~s}), 994(\mathrm{~s}), 836(\mathrm{w}), 797(\mathrm{~s})$, $751(\mathrm{~s}), 715(\mathrm{~s}), 653(\mathrm{w}), 580(\mathrm{~s}) \mathrm{cm}^{-1}$. 
II.8.2 Synthesis of $2^{10}, 2^{20}$-bis(2,6-dichlorophenyl)-4,8,12,16-tetraoxa-2(5,15)zinc(II)porpyhrina-10(4,5)-1,2,3-triazola-1,3(1,2)-dibenzenacyclohexadecaphane-9,11-dione

Column chromatography: (dichloromethane $/$ methanol $=97 / 3, R_{\mathrm{f}}=0.26$ )

Yield: $\quad 26.5 \mathrm{mg}(24.5 \mu \mathrm{mol}, 100 \%)$

m.p.: $201^{\circ} \mathrm{C}$

${ }^{1} \mathrm{H}-\mathrm{NMR}\left(500 \mathrm{MHz}\right.$ (Bruker DRX 500), $\left.\mathrm{CD}_{2} \mathrm{Cl}_{2}, 300 \mathrm{~K}\right): \delta=8.86\left(\mathrm{~d},{ }^{3} \mathrm{~J}=4.5 \mathrm{~Hz}, 4 \mathrm{H}\right)$, $8.65\left(\mathrm{~d},{ }^{3} \mathrm{~J}=4.5 \mathrm{~Hz}, 4 \mathrm{H}\right), 8.24\left(\mathrm{dd},{ }^{3} J=7.3 \mathrm{~Hz},{ }^{4} J=1.6 \mathrm{~Hz}, 2 \mathrm{H}\right), 78.1-7.75(\mathrm{~m}, 4 \mathrm{H})$, $7.73\left(\mathrm{~d},{ }^{3} \mathrm{~J}=8.3 \mathrm{~Hz}, 2 \mathrm{H}\right), 7.68\left(\mathrm{t},{ }^{3} \mathrm{~J}=8.3 \mathrm{~Hz}, 2 \mathrm{H}\right), 7.45\left(\mathrm{td},{ }^{3} \mathrm{~J}=7.3 \mathrm{~Hz},{ }^{4} \mathrm{~J}=0.7 \mathrm{~Hz}\right.$, 2H), $7.30\left(\mathrm{~d},{ }^{3} \mathrm{~J}=8.4 \mathrm{~Hz}, 2 \mathrm{H}\right), 4.05\left(\mathrm{t},{ }^{3} \mathrm{~J}=5.5 \mathrm{~Hz}, 4 \mathrm{H}\right), 2.96\left(\mathrm{t},{ }^{3} \mathrm{~J}=5.5 \mathrm{~Hz}, 4 \mathrm{H}\right), 1.38$ (s, br, 4H) ppm.

MS (MALDI, TOF): $m / z(\%)=1082[\mathrm{M}]^{+}$.

MS: (HR) MALDI

\begin{tabular}{|l|c|c|c|}
\hline calc. & found & Mass. Diff $(\mathrm{ppm})$ & Molecular formula \\
\hline 1083.06732 & 1083.06653 & -0.7 & $\mathrm{C}_{54} \mathrm{H}_{35} \mathrm{Cl}_{4} \mathrm{~N}_{7} \mathrm{O}_{6}$ \\
\hline
\end{tabular}

IR: $\widetilde{v}=2924(w), 2362(w), 2342(w), 1689(m), 1571(m), 1490(m), 1426(m)$, $1335(\mathrm{~m}), 1292(\mathrm{~m}), 1202(\mathrm{~s}), 1110(\mathrm{~m}), 1006(\mathrm{~m}), 994(\mathrm{~s}), 884(\mathrm{~s}), 797(\mathrm{~s}), 775(\mathrm{~m})$, $751(\mathrm{~s}), 713(\mathrm{~s}), 656(\mathrm{w}) \mathrm{cm}^{-1}$. 


\section{Density functional theory calculations}

\section{Computational Methods}

General. All geometry optimizations were performed at the $\operatorname{PBE}^{3}\left(\mathrm{D}^{4}\right) /$ def2SVPD level of density functional theory (DFT). These calculations were carried out with Turbomole $7.2^{5}$ and the usage of resolution-of-identity (RI) with multipole accelerated RI-J (marij) to speed up the calculations.

Conformers of compound 7. Different conformations of compound 7 were calculated to estimate the conformational arrangement of the bridge (fig. 1). With a $c_{2}$ or $c_{\mathrm{s}}$ symmetry constraint the structures seem to be strained and therefore far away from the thermodynamic equilibrium. Without any constraints the different conformational structures are nearly isoenergetic. Even if the carbonyl functions of the esters are in gauche conformation, which should exhibit repulsive forces, there is just a little energetic difference to the thermodynamically most stable structure. This is an indicator of free movement for the bridge in the coordinated and deprotonated HS-form of compound 7.

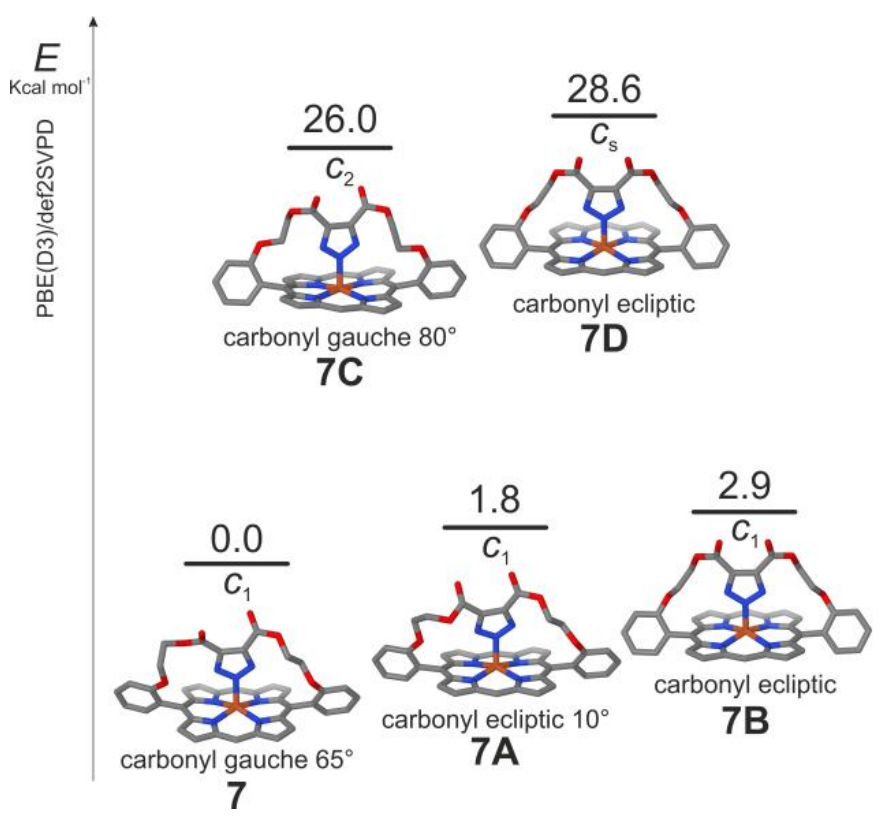

Figure S1: Range of possible conformations with and without symmetry constraints of compound 7 . 


\title{
Cartesian Coordinates of PBE(D3)/def2SVPD optimized structures
}

\section{Compound 7}

\author{
$E_{P B E(D 3) / d e f 2 S V P D}=-5932.023337585$ Hartree \\ Nimag $=0$
}

C $\quad-0.3106531 \quad 0.5182916 \quad 3.1514781$

$\begin{array}{llll}\text { C } & 0.2058141 & -0.8073199 & 3.0150209\end{array}$

$\begin{array}{llll}\text { N } & -0.3031628 & -0.0411785 & 1.0905302\end{array}$

C $0.9095964 \quad-1.7470026 \quad 3.9222154$

C $\quad-0.6344693 \quad 1.2908329 \quad 4.3797480$

$\begin{array}{llll}\text { O } & 0.7094860 & -3.0262979 & 3.4927067\end{array}$

$\begin{array}{lllll}0 & -0.8882056 & 0.7402977 & 5.4342263\end{array}$

O $1.6490153 \quad-1.4336942 \quad 4.8377372$

$\begin{array}{llll}\mathrm{O} & -0.6934321 & 2.6535884 & 4.3155900\end{array}$

N 0.1913008 -1.1101662 1.7034497

N $-\begin{array}{llll}0.6284367 & 0.9517900 & 1.9146439\end{array}$

C $2.9359507-0.0923369-1.2799061$

N $1.7418363 \quad-0.7808605 \quad-1.2028484$

C $4.0439861-1.0197952-1.2346633$

C $3.5038161-2.2742657-1.0893112$

C $2.0677002-2.1145662-1.0605029$

C $3.07259491 .3082289-1.3068934$

C $2.0140364 \quad 2.2341874 \quad-1.2610206$

C $\quad 2.1857700 \quad 3.6680634-1.2185762$

C $0.9325709 \quad 4.2153422 \quad-1.0906163$

$\begin{array}{llll}C & -0.0071841 & 3.1171722 & -1.0661273\end{array}$

N $0.6707975 \quad 1.9191680-1.1848399$

$\begin{array}{llll}\text { Fe } & -0.1476866 & 0.0343336 & -0.9096397\end{array}$

N $-0.9083359-1.8505494-1.3173719$

C $-2.2516939-2.1567310-1.4045555$

C $-2.4410842-3.5801759-1.2450906$

C $-1.2016068-4.1231035-1.0069416$

C $-0.2444933-3.0395383-1.0712904$

C $-1.4089324 \quad 3.2668410 \quad-0.9767684$

C $\quad-2.3266175 \quad 2.2014612-1.1282675$

C $-3.7669456 \quad 2.3370380-1.1034510$

$\begin{array}{llll}\text { C } & -4.2928216 & 1.0852721 & -1.3172073\end{array}$

C $\quad-3.17773540 .1741163-1.4283076$

$\begin{array}{lllll}\text { N } & -1.9907139 & 0.8741785 & -1.3288458\end{array}$ $\begin{array}{llll}\text { C } & -3.3065308 & -1.2270400 & -1.5005923\end{array}$

C $1.1542349-3.1813221-0.9172289$

H $\quad 0.66523525 .2724321 \quad-1.0188269$

H $\quad 3.1453673 \quad 4.1896334-1.2711091$

$\begin{array}{lllll}H & -4.3109876 & 3.2689477 & -0.9315921\end{array}$

$\begin{array}{llll}\text { H } & -5.3473827 & 0.7992521 & -1.3606812\end{array}$

H $-0.9607176-5.1669528-0.7930374$

H $\quad-3.4055823-4.0948745 \quad-1.2706036$

H $5.1001092-0.7420932-1.2921652$

H $4.0278539-3.2302990-1.0089996$

C $4.4598293 \quad 1.8592576 \quad-1.2325616$

C $1.6887153-4.5314415-0.5661541$

$\begin{array}{llll}\text { C } & -1.9488285 & 4.6388079 & -0.7449411\end{array}$

$\begin{array}{llll}\text { C } & -4.6961142 & -1.7786715 & -1.5362935\end{array}$

$\begin{array}{llll}\text { C } & -5.4131716 & -2.0415799 & -0.3460357\end{array}$

$\begin{array}{llll}\text { C } & -6.7170498 & -2.5581873 & -0.3633523\end{array}$

C $-7.3309110-2.8232374-1.5946455$

C $-6.6555055-2.5773335 \quad-2.7970835$

$\begin{array}{llll}\text { C } & -5.3525544 & -2.0606635 & -2.7537858\end{array}$

$\begin{array}{llll}\text { C } & -1.6608760 & 5.3568261 & 0.4475769\end{array}$

$\begin{array}{llll}\text { C } & -2.1842938 & 6.6466988 & 0.6425636\end{array}$

$\begin{array}{llll}\text { C } & -2.9873730 & 7.2461413 & -0.3368875\end{array}$

C $\quad-3.2715240 \quad 6.5542163 \quad-1.5245235$

$\begin{array}{llll}\text { C } & -2.7530807 & 5.2674612 & -1.7201485\end{array}$

C $2.3178295 \quad-4.7525743 \quad 0.6967920$

C $2.7087169-6.0556509 \quad 1.0637510$

C $2.5242665-7.1271903 \quad 0.1775024$

C $1.9489141-6.9173965-1.0831083$

C $1.5347727-5.6252115-1.4385069$

C $5.2167462 \quad 2.1638559-2.3838316$

$\begin{array}{llll}\text { C } & 6.5175017 & 2.6825132 & -2.3044760\end{array}$

$\begin{array}{llll}\text { C } & 7.0860086 & 2.9066252 & -1.0438773\end{array}$

C $6.36962092 .6181490 \quad 0.1255184$

$\begin{array}{llll}\text { C } & 5.0711194 & 2.0999080 & 0.0198874\end{array}$
Cl $-4.5152665 \quad-1.7555581 \quad-4.2606944$

H $\quad-7.1279838-2.7819132-3.7655650$

H $\quad-8.3519847-3.2279254-1.6172796$

Cl $-4.6546663 \quad-1.7156672 \quad 1.1949770$

$\begin{array}{llll}\text { H } & -7.2384893 & -2.7479659 & 0.5828348\end{array}$

H $\quad 1.0722976-5.4439457 \quad-2.4187516$

$\begin{array}{lllll}\text { O } & 2.5216139 & -3.6496296 & 1.4635421\end{array}$

$\begin{array}{llll}\text { H } & 3.1644508 & -6.2409718 & 2.0438351\end{array}$

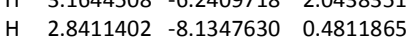

H $\quad 1.8157916 \quad-7.7522172 \quad-1.7840727$

H $\quad 7.0716568 \quad 2.9056352 \quad-3.2243568$

$\begin{array}{llll}\text { Cl } & 4.5114910 & 1.8873305 & -3.9616822\end{array}$

H $\quad 8.1040848 \quad 3.3128804 \quad-0.9710263$

$\begin{array}{llll}H & 6.8072356 & 2.7906859 & 1.1163572\end{array}$

$\begin{array}{lllll}\mathrm{Cl} & 4.1828036 & 1.7396778 & 1.4845208\end{array}$

$\begin{array}{lllll}\text { O } & -0.8106868 & 4.8467031 & 1.4002541\end{array}$

H $\quad-1.9362140 \quad 7.1687794 \quad 1.5765680$

H $\quad-3.3853035 \quad 8.2576143 \quad-0.1741755$

$\begin{array}{llll}H & -3.8889314 & 7.0192879 & -2.3052449\end{array}$

$\begin{array}{lllll}\text { H } & -2.9538807 & 4.7281696 & -2.6562961\end{array}$

$\begin{array}{llll}\text { C } & 2.9239095 & -3.7346712 & 2.8295284\end{array}$

$\begin{array}{llll}\text { C } & 1.7413088 & -3.9816954 & 3.7682034\end{array}$

$\begin{array}{llll}\text { H } & 3.3704422 & -2.7480678 & 3.0492447\end{array}$

H $3.7098786 \quad-4.50162892 .9900059$

H $\quad 1.2770512 \quad-4.9682420 \quad 3.5926389$

H $\quad 2.0829248 \quad-3.9125527 \quad 4.8204454$

$\begin{array}{lllll}\text { C } & -1.3786418 & 3.9353072 & 2.3549609\end{array}$

$\begin{array}{llll}\text { C } & -0.2157367 & 3.4566837 & 3.2207420\end{array}$

$\begin{array}{llll}\mathrm{H} & -1.8501218 & 3.0744173 & 1.8413154\end{array}$

$\begin{array}{llll}H & -2.1360917 & 4.4582348 & 2.9776124\end{array}$

$\begin{array}{llll}H & 0.5213655 & 2.9158015 & 2.6010211\end{array}$

$\begin{array}{llll}H & 0.2801337 & 4.3285793 & 3.6865049\end{array}$

\section{Compound 8}

\section{$E_{P B E(D 3) / d e f 2 S V P D}=-6010.498708821$ Hartree \\ Nimag $=0$}

$\begin{array}{llll}C & -1.0234956 & 0.3965906 & 2.8508492\end{array}$

$\begin{array}{llll}\text { C } & -0.3052202 & -0.8338962 & 2.8315713\end{array}$

$\begin{array}{llll}\text { N } & -0.2387614 & 0.0537012 & 0.8964331\end{array}$

$\begin{array}{llll}\text { C } & 0.0289114 & -1.7823160 & 3.9146426\end{array}$

$\begin{array}{llll}\text { C } & -1.9203385 & 1.0384506 & 3.8554925\end{array}$

$\begin{array}{llll}\text { O } & 0.3961346 & -2.9772622 & 3.3885726\end{array}$

$\begin{array}{llll}0 & -2.8685320 & 0.4570934 & 4.3448980\end{array}$

\begin{tabular}{lllll}
\hline & 0.0219162 & -1.5199678 & 5.1071522
\end{tabular}

$\begin{array}{llll}0 & -1.7090822 & 2.3541280 & 4.1213373\end{array}$

$\begin{array}{llll}\mathrm{N} & 0.1794395 & -0.9991554 & 1.5842621\end{array}$

$\begin{array}{llll}\mathrm{N} & -0.9691442 & 0.9179103 & 1.6127243\end{array}$

$\begin{array}{llll}\text { C } & 3.0013188 & 0.0163517 & -1.5360437\end{array}$

$\begin{array}{llll}\text { N } & 1.8122150 & -0.6807937 & -1.4437808\end{array}$

C $4.1151806-0.9025288-1.4917553$

$\begin{array}{llll}\text { C } & 3.5850628 & -2.1638886 & -1.3598779\end{array}$

$\begin{array}{llll}\text { C } & 2.1488618 & -2.0167416 & -1.3312472\end{array}$

C $3.1255189 \quad 1.4152710 \quad-1.6307713$

C $2.0588888 \quad 2.3329635-1.6238462$

C $2.2154931 \quad 3.7667236 \quad-1.7074349$

$\begin{array}{llll}\text { C } & 0.9590966 & 4.3115650 & -1.6023032\end{array}$

C $0.0322537 \quad 3.2121298-1.4615925$

N $0.7207249 \quad 2.0130232 \quad-1.4903490$

Fe $-0.0625870 \quad 0.1316684-1.1012902$

$\begin{array}{llll}N & -0.8500555 & -1.7687293 & -1.4303607\end{array}$

C $-2.1914327-2.0868743-1.3582006$

$\begin{array}{llll}\text { C } & -2.3531580 & -3.5163914 & -1.2171564\end{array}$

$\begin{array}{llll}\text { C } & -1.0914389 & -4.0560587 & -1.1678747\end{array}$

C $-0.1579412-2.9571962-1.2923400$

C $-1.3660164 \quad 3.3593065-1.3351296$

$\begin{array}{lllll}C & -2.2765081 & 2.2797134 & -1.3328591\end{array}$

C $-3.7081289 \quad 2.4158213 \quad-1.1714215$
$\begin{array}{lllll}C & -4.2366107 & 1.1486187 & -1.1976132\end{array}$

C $-3.12867520 .2355976-1.3540024$

$\begin{array}{lllll}\text { N } & -1.9452489 & 0.9426358 & -1.4524037\end{array}$

C $-3.2558909-1.1658744 \quad-1.3257144$

C $1.2442956-3.0930154-1.2298126$

$\begin{array}{lllll}\text { H } & 0.6800517 & 5.3680919 & -1.6149467\end{array}$

H $3.1682058 \quad 4.2905706-1.8242753$

$\begin{array}{lllll}\text { H } & -4.2404953 & 3.3599810 & -1.0332576\end{array}$

$\begin{array}{llll}\text { H } & -5.2847028 & 0.8546145 & -1.0946188\end{array}$

H $-0.8124202-5.1047198 \quad-1.0381318$

$\begin{array}{llll}\text { H } & -3.3120197 & -4.0363425 & -1.1403221\end{array}$

H $\quad 5.1691325-0.6166397-1.5496076$

H $\quad 4.1157574-3.1174304-1.2932476$

C $4.5090323 \quad 1.9790850-1.6718471$

C $1.7972616-4.4675148-1.0279295$

C $\quad-1.9185626 \quad 4.7393878-1.1975029$

C $-4.6262691-1.7203734-1.1009216$

$\begin{array}{llll}\text { C } & -5.1334424 & -1.8861753 & 0.2102022\end{array}$

C $-6.4144771-2.4090552 \quad 0.4439339$

$\begin{array}{llll}\text { C } & -7.2143076 & -2.7789003 & -0.6451979\end{array}$

$\begin{array}{llll}\text { C } & -6.7483466 & -2.6294374 & -1.9581087\end{array}$

$\begin{array}{llll}\text { C } & -5.4656615 & -2.1033989 & -2.1684903\end{array}$

C $-1.6174331 \quad 5.5409188-0.0636202$

$\begin{array}{llll}\text { C } & -2.1584313 & 6.8331982 & 0.0535116\end{array}$

$\begin{array}{llll}\text { C } & -2.9948442 & 7.3480401 & -0.9463403\end{array}$

C $-3.29547386 .5694366-2.0750886$

$\begin{array}{lllll}\text { C } & -2.7588924 & 5.2802954 & -2.1937423\end{array}$

$\begin{array}{llll}\text { C } & 2.3381681 & -4.8449840 & 0.2349727\end{array}$

$\begin{array}{llll}\text { C } & 2.7988049 & -6.1645720 & 0.4276198\end{array}$

$\begin{array}{llll}\text { C } & 2.7447897 & -7.0926957 & -0.6236719\end{array}$

C $2.2294508 \quad-6.7271826-1.8740887$ $\begin{array}{llll}\text { C } & 1.7575828 & -5.4184977 & -2.0615886\end{array}$

$\begin{array}{llll}\text { C } & 5.2097834 & 2.1582692 & -2.8846417\end{array}$

C $\quad 6.5079557 \quad 2.6876277 \quad-2.9246803$

C $7.1331618 \quad 3.0523173 \quad-1.7252922$

$\begin{array}{llll}\text { C } & 6.4744316 & 2.8922327 & -0.4990410\end{array}$

C $5.1771392 \quad 2.3603355-0.4859079$

$\begin{array}{llll}\text { Cl } & -4.8898221 & -1.9152367 & -3.8123345\end{array}$

H $\quad-7.3678871-2.9151395 \quad-2.8169518$

$\begin{array}{llll}H & -8.2178829 & -3.1892487 & -0.4680783\end{array}$

$\begin{array}{clll}\mathrm{Cl} & -4.1503198 & -1.4219544 & 1.5769662\end{array}$

H $\quad-6.7721956-2.5202714 \quad 1.4748081$

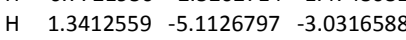

$\begin{array}{llll}\text { O } & 2.3663497 & -3.8720166 & 1.1819953\end{array}$

$\begin{array}{llll}\text { H } & 3.1953112 & -6.4786520 & 1.4001834\end{array}$

H $3.1094005 \quad-8.1154486-0.4530523$

H $\quad 2.1884294 \quad-7.4529206-2.6973141$

H $\quad 7.0159645 \quad 2.8100405 \quad-3.8890662$

$\begin{array}{lllll}\text { Cl } & 4.4352250 & 1.7109873 & -4.3885811\end{array}$

H $8.1497228 \quad 3.4682795 \quad-1.7463589$

H $\quad 6.9559840 \quad 3.1752179 \quad 0.4449274$

$\begin{array}{llll}\text { Cl } & 4.3627000 & 2.1597349 & 1.0505935\end{array}$

$\begin{array}{lllll}\text { O } & -0.7386003 & 5.0984468 & 0.8935999\end{array}$

H $\quad-1.8993545 \quad 7.4246131 \quad 0.9421211$

$\begin{array}{lllll}H & -3.4077427 & 8.3616344 & -0.8453942\end{array}$

$\begin{array}{lllll}H & -3.9424672 & 6.9676639 & -2.8687747\end{array}$

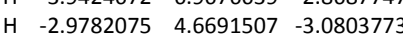

$\begin{array}{llll}\text { C } \quad 2.8596232 & -4.1617767 & 2.4928209\end{array}$

$\begin{array}{llll}\text { C } & 1.8291646 & -4.8830360 & 3.3847875\end{array}$

H $3.1092906-3.1696240 \quad 2.9109010$

H $3.8060254 \quad-4.7363204 \quad 2.4185466$

$\begin{array}{llll}\text { H } & 1.1518780 & -5.4752884 & 2.7393300\end{array}$ 
$\begin{array}{llll}\text { H } & 2.3433382 & -5.6044033 & 4.0509718\end{array}$

$\begin{array}{llll}\text { C } & -1.2751546 & 4.2829584 & 1.9556562\end{array}$

$\begin{array}{llll}\text { C } & -0.0868739 & 3.6175830 & 2.6346968\end{array}$

H $\quad-1.9572057 \quad 3.5143490 \quad 1.5380615$

H $\quad-1.8558589 \quad 4.9234418 \quad 2.6555512$
$\begin{array}{llll}H & 0.3438405 & 2.8881747 & 1.9270380\end{array}$ H $\quad 0.6868028 \quad 4.3903961 \quad 2.8180922$ $\begin{array}{llll}\text { C } & -0.3958318 & 2.9622995 & 3.9852237\end{array}$ H $\quad-0.3967660 \quad 3.7238742 \quad 4.7863272$

H $\quad 0.3796059 \quad 2.2110176 \quad 4.2331505$
C $\quad 1.0140519 \quad-3.9355650 \quad 4.2600951$ $\begin{array}{llll}\text { H } & 1.6521431 & -3.3994789 & 4.9918404\end{array}$ H $\quad 0.2340880 \quad-4.4758587 \quad 4.8327717$

\section{Compound 7 open}

\section{$E_{P B E(D 3) / d e f 2 S V P D}=-6241.519261829$ Hartree Nimag $=0$}

$\begin{array}{llll}\text { C } & -0.8352190 & 0.1716480 & 2.4068859\end{array}$

$\begin{array}{llll}\text { C } & 0.5478674 & -0.1777314 & 2.3702114\end{array}$

$\begin{array}{llll}\text { N } & -0.3105460 & -0.4948281 & 0.4445284\end{array}$

C $1.6232725-0.0052321 \quad 3.3752266$

$\begin{array}{llll}\text { C } & -1.6583088 & 0.5816091 & 3.5779839\end{array}$

$\begin{array}{llll}\text { O } & 2.5051912 & -1.0349283 & 3.3242002\end{array}$

$\begin{array}{llll}0 & -1.3817772 & 0.2111336 & 4.7061029\end{array}$

$\begin{array}{llll}0 & 1.7410235 & 0.9591031 & 4.1121599\end{array}$

$\begin{array}{lllll}\mathrm{O} & -2.7446049 & 1.3674365 & 3.3810581\end{array}$

$\begin{array}{llll}\text { N } & 0.8375748 & -0.5863367 & 1.1222514\end{array}$

$\begin{array}{llll}N & -1.3364791 & -0.0575763 & 1.1791736\end{array}$

C $2.6226755-1.0911748-2.1858068$

N $1.3076204-1.5016758-2.0912002$

C $3.4905384-2.2433206-2.2689097$

C $2.6876253-3.3544425 \quad-2.1667631$

$\begin{array}{llll}\text { C } & 1.3282173 & -2.8796400 & -2.0515917\end{array}$

$\begin{array}{llll}\text { C } & 3.0706504 & 0.2396688 & -2.0971401\end{array}$

C $\quad 2.2425307 \quad 1.3575002-1.8844458$

C $2.7228721 \quad 2.7033114-1.6617218$

$\begin{array}{llll}\text { C } & 1.6228864 & 3.4757632 & -1.3780291\end{array}$

$\begin{array}{llll}C & 0.4706019 & 2.6057006 & -1.4499399\end{array}$

N $0.8679078 \quad 1.3247386 \quad-1.7717930$

Fe $-0.3312170 \quad-0.3633463 \quad-1.5643153$

$\begin{array}{llll}N & -1.5333986 & -1.9630197 & -2.1019524\end{array}$

C $-2.9106224-1.9715848-2.1736309$

C $-3.3941427-3.3346963-2.1453945$

C $-2.2943360-4.1460668-1.9996632$

C $-1.1338752-3.2821556-1.9929103$

C $-0.8599181 \quad 3.0334807-1.2510171$

$\begin{array}{llll}C & -1.9932579 & 2.2283085 & -1.4949065\end{array}$

$\begin{array}{llll}\text { C } & -3.3615546 & 2.6917559 & -1.4262518\end{array}$

$\begin{array}{lllll}\text { C } & -4.1625997 & 1.6214327 & -1.7458797\end{array}$

C $\quad-3.2867478 \quad 0.4919960 \quad-1.9648072$

$\begin{array}{lllll}N & -1.9713460 & 0.8874355 & -1.8347064\end{array}$

C $-3.7359198 \quad-0.8310208$ - 2.1484645

$\begin{array}{llll}\text { C } & 0.2030574 & -3.7223935 & -1.9110900\end{array}$

H $\quad 1.5858146 \quad 4.5435336-1.1462686$

$\begin{array}{lllll}H & 3.7708863 & 3.0127402 & -1.7049974\end{array}$

H $\quad-3.6670973 \quad 3.7089286 \quad-1.1690734$

H $\quad-5.2543280 \quad 1.5917370 \quad-1.8017246$
H $\quad-2.2669603 \quad-5.2351265 \quad-1.9087649$

H $\quad-4.4458771-3.6305859-2.1959802$

H $4.5785460-2.2047862-2.3730162$

H $\quad 2.9836702-4.4070462 \quad-2.1740657$

C $4.54847780 .4665919 \quad-2.0739948$

C $0.4719170 \quad-5.1406368 \quad-1.5343076$

$\begin{array}{lllll}\text { C } & -1.0674634 & 4.4115766 & -0.7162872\end{array}$

C $-5.2140880-1.0539715 \quad-2.1654934$

C $-5.9456499-1.2139221 \quad-0.9653369$

C $-7.3324284-1.4230534-0.9621352$

$\begin{array}{llll}\text { C } & -8.0177068 & -1.4792851 & -2.1830882\end{array}$

C $-7.3314026-1.3272683-3.3947629$

$\begin{array}{llll}\text { C } & -5.9448507 & -1.1172565 & -3.3717148\end{array}$

C $\quad-0.7129874 \quad 4.6802709 \quad 0.6375189$

C $\quad-0.9188758 \quad 5.9683165 \quad 1.1699312$

$\begin{array}{llll}\text { C } & -1.4734937 & 6.9787618 & 0.3704149\end{array}$

C $-1.8247703 \quad 6.7264233 \quad-0.9635233$

C $-1.6161536 \quad 5.4442389-1.4951924$

C $0.9531826-5.3753669-0.2128373$

$\begin{array}{llll}\text { C } & 1.2270396 & -6.6894919 & 0.2116250\end{array}$

C $1.0182547-7.7634387-0.6695299$

C $0.5419193 \quad-7.5446861 \quad-1.9695850$

$\begin{array}{llll}\text { C } & 0.2728611 & -6.2319480 & -2.3929383\end{array}$

C $5.2810116 \quad 0.7729589 \quad-3.2406763$

C $6.66706720 .9872900-3.2167638$

C $7.35041190 .8944614 \quad-1.9973966$

C $6.6625592 \quad 0.5920584 \quad-0.8147169$

C $5.2767581 \quad 0.3832718 \quad-0.8637710$

$\begin{array}{llll}\mathrm{Cl} & -5.0962026 & -0.9238924 & -4.8904879\end{array}$

H $\quad-7.8593578-1.3698218-4.3552593$

H $\quad-9.1037730-1.6441729-2.1899998$

$\begin{array}{lllll}\mathrm{Cl} & -5.0967837 & -1.1484060 & 0.5620253\end{array}$

H $\quad-7.8612038-1.5425866-0.0086351$

H $\quad-0.0896848-6.0384334-3.4123222$

$\begin{array}{lllll}\text { O } & 1.1015974 & -4.2666203 & 0.5552258\end{array}$

H $\quad 1.5931538-6.8794788 \quad 1.2278261$

H $\quad 1.2332373 \quad-8.7853735 \quad-0.3266136$

$\begin{array}{llll}\text { H } & 0.3850970 & -8.3882388 & -2.6551527\end{array}$

H $\quad 7.1971414 \quad 1.2237500-4.1474199$

$\begin{array}{lllll}\text { Cl } & 4.4356962 & 0.8878942 & -4.7688625\end{array}$
$\begin{array}{llll}H & 8.4358877 & 1.0605888 & -1.9680155\end{array}$

H $\quad \begin{array}{llll}7.1875484 & 0.5162614 & 0.1452839\end{array}$

$\begin{array}{lllll}\mathrm{Cl} & 4.4202411 & 0.0003502 & 0.6102818\end{array}$

$\begin{array}{llll}\text { O } & -0.1955839 & 3.6382496 & 1.3353588\end{array}$

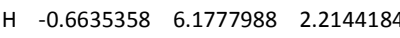

$\begin{array}{llll}H & -1.6326585 & 7.9763447 & 0.8035952\end{array}$

$\begin{array}{llll}H & -2.2527356 & 7.5207947 & -1.5896459\end{array}$

$\begin{array}{llll}\text { H } & -1.8743063 & 5.2281897 & -2.5412620\end{array}$

C $4.5767993 \quad-2.1059669 \quad 3.7847919$

C $3.6982292 \quad-0.8978821 \quad 4.1196129$

H $4.6194195 \quad-2.2280571 \quad 2.6781264$

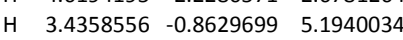

H $\quad 4.1969427 \quad 0.0567006 \quad 3.8606929$

$\begin{array}{llll}\text { C } & -3.9604867 & 3.1645476 & 2.3565251\end{array}$

$\begin{array}{llll}\text { C } & -3.0879583 & 1.9571928 & 2.1053453\end{array}$

H $-4.8052546 \quad 2.8991721 \quad 3.0343601$

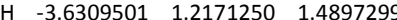

$\begin{array}{llll}H & -2.1770857 & 2.2601565 & 1.5608573\end{array}$

$\begin{array}{llll}\text { H } & -4.3953715 & 3.4757671 & 1.3763551\end{array}$

$\begin{array}{llll}\text { C } & 0.1631028 & 3.8269758 & 2.7081409\end{array}$

$\begin{array}{llll}H & 0.5880956 & 2.8705214 & 3.0522842\end{array}$

$\begin{array}{lllll}H & 0.9314012 & 4.6206083 & 2.8055065\end{array}$

H $\quad-0.7319138 \quad 4.0795160 \quad 3.3091407$

$\begin{array}{llll}\text { O } & -3.1833569 & 4.2110153 & 2.9101038\end{array}$

$\begin{array}{llll}\text { C } & -3.9054671 & 5.4138797 & 3.0648652\end{array}$

H $\quad-3.2209250 \quad 6.1556569 \quad 3.5137986$

$\begin{array}{llll}H & -4.2599436 & 5.8035626 & 2.0825911\end{array}$

H $\quad-4.7847862 \quad 5.2809466 \quad 3.7357298$

$\begin{array}{llll}\text { C } & 1.6506962 & -4.3726163 & 1.8623441\end{array}$

$\begin{array}{llll}H & 0.9948337 & -4.9722797 & 2.5272277\end{array}$

H $\quad 2.6628902-4.8282402 \quad 1.8344745$

$\begin{array}{llll}H & 1.7239769 & -3.3406106 & 2.2429081\end{array}$

H $\quad 4.1287845 \quad-3.0249080 \quad 4.2098083$

$\begin{array}{llll}\text { O } & 5.8752010 & -1.9876283 & 4.3403074\end{array}$

$\begin{array}{llll}\text { C } & 6.7650293 & -1.1942204 & 3.5724307\end{array}$

H $\quad 6.4286384-0.1373444 \quad 3.4838616$

H $7.7408399-1.2127542 \quad 4.0896104$

H $\quad 6.8865027 \quad-1.6072053 \quad 2.5451285$

\section{Compound 7B}

\section{$E_{\mathrm{PBE}(\mathrm{D} 3) / \mathrm{det2SVPD}}=-5932.018762245$ Hartree} Nimag $=0$

C $\quad 1.2971629 \quad 2.6390810 \quad 1.2499911$

$\begin{array}{llll}\text { N } & 1.2492961 & 1.2736038 & 1.4542851\end{array}$

C $1.3496443 \quad 3.3285777 \quad 2.5180218$

C $1.3276743 \quad 2.3642126 \quad 3.4946346$

C $1.2412918 \quad 1.0859302 \quad 2.8244910$

C $1.2825421 \quad 3.2831453 \quad-0.0000149$

C $1.2973161 \quad 2.6390825-1.2500151$

$\begin{array}{llll}\text { C } & 1.3498512 & 3.3285439 & -2.5180612\end{array}$

$\begin{array}{llll}\text { C } & 1.3278845 & 2.3641475 & -3.4946475\end{array}$

C $1.2415831 \quad 1.0858848 \quad-2.8244571$

N $1.24942351 .2736085 \quad-1.4542692$

$\begin{array}{llll}\text { Fe } & 0.8426145 & -0.1538277 & -0.0000012\end{array}$

$\begin{array}{llll}\mathrm{N} & 1.1253808 & -1.5984485 & 1.4622341\end{array}$

$\begin{array}{llll}\text { C } & 0.9415532 & -2.9524563 & 1.2526786\end{array}$

$\begin{array}{llll}\text { C } & 0.7918670 & -3.6305832 & 2.5182681\end{array}$

$\begin{array}{llll}\text { C } & 0.8675161 & -2.6694550 & 3.4971616\end{array}$

C $1.0706360-1.4020015 \quad 2.8300601$

$\begin{array}{llll}\text { C } & 1.1299639 & -0.1533453 & -3.4887151\end{array}$

$\begin{array}{llll}\text { C } & 1.0707600 & -1.4020382 & -2.8299968\end{array}$

$\begin{array}{llll}\text { C } & 0.8672999 & -2.6694420 & -3.4970930\end{array}$

C $0.7916395-3.6305753-2.5182044$

C $0.9415333-2.9524848 \quad-1.2526222$

N $1.1254940 \quad-1.5984966 \quad-1.4621780$

$\begin{array}{llll}\text { C } & 0.8486249 & -3.5877798 & 0.0000338\end{array}$ $\begin{array}{llll}\text { C } & 1.1296869 & -0.1532986 & 3.4887855\end{array}$

H $\quad 1.3562612 \quad 2.5045583 \quad-4.5777295$

$\begin{array}{llll}\text { H } & 1.4012697 & 4.4134322 & -2.6438964\end{array}$

H $\quad 0.7707681-2.8042760-4.5771549$

H $\quad 0.6307213-4.7050653 \quad-2.6418046$

$\begin{array}{llll}H & 0.7712337 & -2.8043381 & 4.5772440\end{array}$

H $0.6311011 \quad-4.7050961 \quad 2.6418734$

H $\quad 1.4010584 \quad 4.4134683 \quad 2.6438338$

$\begin{array}{llll}\text { H } & 1.3561675 & 2.5046607 & 4.5777111\end{array}$

$\begin{array}{llll}\text { C } & 1.1280414 & 4.7690082 & -0.0000109\end{array}$

$\begin{array}{llll}\text { C } & 1.0284686 & -0.1395727 & 4.9780205\end{array}$

C $1.0286115-0.1396237-4.9779453$

C $0.5059773 \quad-5.04283920 .0000610$

$\begin{array}{llll}\text { C } & -0.8455170 & -5.4609728 & 0.0000763\end{array}$

$\begin{array}{llll}\text { C } & -1.2002396 & -6.8178343 & 0.0000514\end{array}$

$\begin{array}{llll}\text { C } & -0.1896028 & -7.7883911 & 0.0001064\end{array}$

$\begin{array}{llll}\text { C } & 1.1610666 & -7.4155824 & 0.0000296\end{array}$

$\begin{array}{llll}\text { C } & 1.4914244 & -6.0526547 & 0.0000545\end{array}$

$\begin{array}{llll}\text { C } & -0.0700516 & 0.4697632 & -5.6433736\end{array}$

$\begin{array}{llll}\text { C } & -0.1239702 & 0.4859849 & -7.0479783\end{array}$

$\begin{array}{llll}\text { C } & 0.8986479 & -0.0976551 & -7.8079254\end{array}$

$\begin{array}{llll}\text { C } & 1.9855493 & -0.7092789 & -7.1643524\end{array}$

$\begin{array}{llll}\text { C } 2.0433417 & -0.7248036 & -5.7643739\end{array}$

$\begin{array}{llll}\text { C } & -0.0703125 & 0.4694752 & 5.6435926\end{array}$

$\begin{array}{llll}\text { C } & -0.1240019 & 0.4857551 & 7.0482098\end{array}$
C $0.8990025 \quad-0.0973710 \quad 7.8080284$

$\begin{array}{llll}\text { C } & 1.9859529 & -0.7087656 & 7.1643212\end{array}$

C $2.0435067 \quad-0.7243931 \quad 5.7643342$

$\begin{array}{llll}\text { C } & 2.2246323 & 5.6567517 & 0.0001289\end{array}$

C $\quad 2.0542082 \quad 7.0490584 \quad 0.0002122$

C $\quad 0.7567861 \quad 7.5774910 \quad 0.0002698$

$\begin{array}{llll}\text { C } & -0.3608177 & 6.7317550 & 0.0000411\end{array}$

$\begin{array}{lllll}\text { C } & -0.1640497 & 5.3441320 & -0.0001268\end{array}$

$\begin{array}{llll}C l & 3.1806348 & -5.5913339 & 0.0001265\end{array}$

H $1.9578501 \quad-8.1693924-0.0000975$

$\begin{array}{llll}H & -0.4592206 & -8.8533176 & 0.0002757\end{array}$

$\begin{array}{cccc}\mathrm{Cl} & -2.1107705 & -4.2545199 & 0.0001480\end{array}$

H $-2.2597829-7.1014428-0.0000648$

H $\quad 2.9005079 \quad-1.1829481 \quad 5.2514271$

$\begin{array}{llll}H & -0.9867870 & 0.9699619 & 7.5249096\end{array}$

$\begin{array}{llll}H & 0.8447554 & -0.0740140 & 8.9054460\end{array}$

$\begin{array}{lllll}H & 2.7948705 & -1.1657828 & 7.7505696\end{array}$

$\begin{array}{lllll}\mathrm{H} & 2.9333481 & 7.7049951 & 0.0001797\end{array}$

Cl $3.8476343 \quad 5.0024895 \quad 0.0001584$

H $\quad 0.6135196 \quad 8.6666466 \quad 0.0005774$

H $-1.3798101 \quad 7.1372623 \quad-0.0000149$

Cl $-1.5635226 \quad 4.2898803 \quad-0.0007452$

$\begin{array}{llll}\text { O } & -1.0640572 & 1.1239143 & -4.9558706\end{array}$

$\begin{array}{llll}\text { H } & -0.9865589 & 0.9706422 & -7.5245734\end{array}$

H $\quad 0.8441158 \quad-0.0745609 \quad-8.9053344$ 
$\begin{array}{llll}\text { H } & 2.7942586 & -1.1665334 & -7.7507027\end{array}$

H $2.9002853-1.1835929-5.2515874$

$\begin{array}{llll}\text { C } & -2.0106030 & 0.3134002 & -4.2395229\end{array}$

$\begin{array}{llll}\text { C } & -2.8737882 & 1.2676380 & -3.4181351\end{array}$

$\begin{array}{llll}\text { H } & -1.4977242 & -0.4062657 & -3.5725563\end{array}$

$\begin{array}{llll}H & -2.6481357 & -0.2431656 & -4.9589981\end{array}$

$\begin{array}{llll}H & -2.2947598 & 1.7068973 & -2.5865199\end{array}$

$\begin{array}{llll}H & -3.2377667 & 2.0814732 & -4.0728365\end{array}$

$\begin{array}{llll}\text { O } & -1.0645413 & 1.1234751 & 4.9562822\end{array}$
$\begin{array}{llll}C & -2.0106690 & 0.3129250 & 4.2394233\end{array}$

H $-1.4974266-0.4065400 \quad 3.5725226$

$\begin{array}{llll}\text { H } & -2.6483889 & -0.2438650 & 4.9585600\end{array}$

C $-2.8737041 \quad 1.2671847 \quad 3.4178978$

$\begin{array}{llll}\text { H } & -2.2945805 & 1.7063091 & 2.5862758\end{array}$

H $\quad-3.2376207 \quad 2.0811097 \quad 4.0725220$

$\begin{array}{lllll}\text { O } & -4.0540055 & 0.5850661 & 2.9612342\end{array}$

$\begin{array}{llll}0 & -4.0540358 & 0.5854078 & -2.9614853\end{array}$

$\begin{array}{llll}\text { C } & -4.2883410 & 0.0678571 & -1.7155166\end{array}$
C $\quad-4.2884005 \quad 0.0677793 \quad 1.7151805$

$\begin{array}{llll}\text { O } & -5.3920070 & -0.3765039 & 1.4795119\end{array}$

$\begin{array}{llll}\text { O } & -5.3918983 & -0.3765901 & -1.4799373\end{array}$

$\begin{array}{lllll}\text { C } & -3.1763045 & 0.0384707 & -0.7191259\end{array}$

$\begin{array}{llll}\text { C } & -3.1763367 & 0.0384470 & 0.7188303\end{array}$

$\begin{array}{llll}\mathrm{N} & -1.1678024 & -0.1428815 & -0.0000980\end{array}$

N $-1.8929801 \quad-0.07218891 .1121509$

N $-1.8929252-0.0721585-1.1123849$

\section{Compound 7A}

\section{$E_{P B E(D 3) / d e f 2 S V P D}=-5932.020396450$ Hartree Nimag $=0$}

$\begin{array}{llll}\text { C } & -0.0618763 & 0.7032756 & 3.1880528\end{array}$

$\begin{array}{llll}C & 0.5939569 & -0.5577381 & 3.0369533\end{array}$

$\begin{array}{llll}\text { N } & -0.3336660 & -0.0104465 & 1.1920534\end{array}$

C $1.4076171 \quad-1.4674010 \quad 3.8933781$

$\begin{array}{llll}\text { C } & -0.1835767 & 1.5996973 & 4.3678742\end{array}$

O $1.1624543 \quad-1.8428692 \quad 5.0222258$

$\begin{array}{llll}0 & -0.1662430 & 1.1832408 & 5.5096230\end{array}$

$\begin{array}{llll}\text { O } & 2.4699429 & -1.8868152 & 3.1438885\end{array}$

$\begin{array}{llll}O & -0.3606439 & 2.9389990 & 4.1543583\end{array}$

$\begin{array}{llll}\text { N } & 0.4028144 & -0.9574590 & 1.7676072\end{array}$

$\begin{array}{llll}\mathrm{N} & -0.6190200 & 1.0081785 & 1.9966365\end{array}$

C $2.8520716-0.1466042 \quad-1.0641018$

N $1.6575844 \quad-0.8355968-1.0940296$

C $3.9580424-1.0735778-0.9652590$

C $3.4137802-2.3320810-0.9104090$

C $1.9780954-2.1731088-0.9750022$

$\begin{array}{llll}C & 2.9924201 & 1.2530084 & -1.0725906\end{array}$

C $1.9363067 \quad 2.1817091-1.1143849$

C $2.1147524 \quad 3.6152287 \quad-1.1178075$

C $0.8601067 \quad 4.1721426-1.0857258$

C $-0.0863970 \quad 3.0794383 \quad-1.0567675$

$\begin{array}{lllll}\mathrm{N} & 0.5893659 & 1.8739847 & -1.1000164\end{array}$

Fe $-0.2402056-0.0112492 \quad-0.8141741$

N $\quad-1.0030254 \quad-1.8905367 \quad-1.2351124$

$\begin{array}{llll}\text { C } & -2.3458882 & -2.1951498 & -1.3403847\end{array}$

C $-2.5312639-3.6257263-1.2713390$

C $-1.2881808-4.1805956-1.0843419$

C $-0.3345572-3.0921237-1.0761264$

$\begin{array}{llll}\text { C } & -1.4884945 & 3.2391289 & -0.9930518\end{array}$

C $-2.4082896 \quad 2.1683975-1.0883806$

C $-3.8477002 \quad 2.3075038-1.0475148$

C $\quad-4.3790033 \quad 1.0511569 \quad-1.2158165$

$\begin{array}{llll}C & -3.2680167 & 0.1356500 & -1.3208418\end{array}$

$\begin{array}{lllll}\text { N } & -2.0771463 & 0.8337182 & -1.2463728\end{array}$
C $-3.4009584-1.2637516-1.4032208$

C $1.0634056-3.2446519 \quad-0.9279479$

$\begin{array}{lllll}H & 0.5966728 & 5.2322258 & -1.0763267\end{array}$

H $3.0789397 \quad 4.1302173 \quad-1.1390966$

$\begin{array}{llll}H & -4.3876027 & 3.2455059 & -0.8977825\end{array}$

H $\quad-5.4345151 \quad 0.7662680-1.2379222$

H $-1.0403218-5.2367190-0.9544700$

H $\quad-3.4948982-4.1397224-1.3267891$

H $\quad 5.0140850-0.7919543 \quad-0.9341796$

H $\quad 3.9334184-3.2900024 \quad-0.8268792$

C $4.3694248 \quad 1.8020595 \quad-0.8828875$

$\begin{array}{llll}\text { C } & 1.5881037 & -4.6285149 & -0.7115133\end{array}$

$\begin{array}{lllll}\text { C } & -2.0299650 & 4.6238585 & -0.8580050\end{array}$

C $-4.7906768-1.8124628 \quad-1.4638673$

$\begin{array}{llll}\text { C } & -5.5137912 & -2.1198405 & -0.2884633\end{array}$

$\begin{array}{llll}\text { C } & -6.8175108 & -2.6353849 & -0.3313013\end{array}$

C $-7.4251982-2.8541263-1.5746088$

C $\quad-6.7439578-2.5628728-2.7636062$

$\begin{array}{llll}\text { C } & -5.4414328 & -2.0480447 & -2.6946029\end{array}$

$\begin{array}{llll}\text { C } & -1.7342014 & 5.4515530 & 0.2615315\end{array}$

$\begin{array}{llll}\text { C } & -2.2566546 & 6.7554844 & 0.3329809\end{array}$

C $-3.0664068 \quad 7.2613466 \quad-0.6919939$

C $\quad-3.3632131 \quad 6.4595138$-1.8047625

$\begin{array}{llll}\text { C } & -2.8459794 & 5.1599663 & -1.8786685\end{array}$

C $2.1492453 \quad-5.0151897 \quad 0.5415826$

$\begin{array}{llll}\text { C } & 2.5107146 & -6.3601061 & 0.7581141\end{array}$

C $2.3684186-7.3071418-0.2662744$

C $1.8638350-6.9305962-1.5184136$

C $1.4753425-5.5990291-1.7250962$

C $5.2212646 \quad 2.1138043 \quad-1.9634323$

C $6.5095254 \quad 2.6338669-1.7701625$

$\begin{array}{llll}\text { C } & 6.9678257 & 2.8506859 & -0.4641666\end{array}$

C $6.1551731 \quad 2.5532854 \quad 0.6382418$

$\begin{array}{lll}\text { C } 4.8712292 & 2.0344549 & 0.4191332\end{array}$
Cl $-4.5970514 \quad-1.6875410 \quad-4.1851129$

H $\quad-7.2116707 \quad-2.7308793 \quad-3.7414036$

$\begin{array}{llll}\text { H } & -8.4460159 & -3.2577841 & -1.6175577\end{array}$

$\begin{array}{llll}\mathrm{Cl} & -4.7637324 & -1.8487425 & 1.2672580\end{array}$

H $\quad-7.3436527 \quad-2.86022020 .6045402$

H $\quad 1.0577470 \quad-5.2893664 \quad-2.6932589$

H $\quad 2.9128964 \quad-6.6754870 \quad 1.7282172$

H $2.6624546 \quad-8.3490748 \quad-0.0775369$

H $\quad 1.7627034 \quad-7.6673560-2.3265300$

$\begin{array}{llll}H & 7.1398049 & 2.8635992 & -2.6379596\end{array}$

Cl $4.6539060 \quad 1.8454019$-3.5973835

H $\quad 7.9751031 \quad 3.2580752 \quad-0.3022548$

H $\quad 6.5065710 \quad 2.7191798 \quad 1.6638296$

$\begin{array}{llll}\mathrm{Cl} & 3.8622486 & 1.6572305 & 1.7984467\end{array}$

$\begin{array}{llll}\text { O } & -0.8678258 & 5.0683005 & 1.2547869\end{array}$

$\begin{array}{llll}H & -2.0024155 & 7.3609269 & 1.2132601\end{array}$

$\begin{array}{lllll}H & -3.4622050 & 8.2841767 & -0.6212684\end{array}$

H $\quad-3.9879357 \quad 6.8469757 \quad-2.6211311$

H $\quad-3.0538201 \quad 4.5347478 \quad-2.7581348$

$\begin{array}{llll}\text { C } & -1.2860698 & 4.0475579 & 2.1771424\end{array}$

$\begin{array}{llll}\text { C } & -0.0284368 & 3.6385949 & 2.9407323\end{array}$

H $\quad-1.7179232 \quad 3.1775064 \quad 1.6476614$

$\begin{array}{llll}H & -2.0390650 & 4.4633465 & 2.8801484\end{array}$

$\begin{array}{lllll}H & 0.6447120 & 3.0455957 & 2.2957669\end{array}$

H $\quad 0.5044942 \quad 4.5532745 \quad 3.2625414$

$\begin{array}{lllll}\text { O } & 2.3296142 & -4.0209821 & 1.4531975\end{array}$

$\begin{array}{llll}\text { C } & 2.4686792 & -4.2960357 & 2.8468001\end{array}$

$\begin{array}{llll}\text { H } & 3.1044067 & -5.1845397 & 3.0392728\end{array}$

H $\quad 1.4632073 \quad-4.4837784 \quad 3.2785265$

C $3.1361386-3.09600393 .5109125$

H $4.1733863 \quad-2.9827911 \quad 3.1476942$

H $3.1342897 \quad-3.2332725 \quad 4.6105534$

\section{Compound 7D}

\section{$E_{P B E(D 3) / d e f 2 S V P D}=-5931.977759736$ Hartree Nimag $=0$}

C $\quad 1.3009691 \quad 2.6480995 \quad 1.2561766$

$\begin{array}{llll}\mathrm{N} & 1.2943650 & 1.2886071 & 1.4671499\end{array}$

C $1.3540991 \quad 3.3533560 \quad 2.5345576$

C $1.3703333 \quad 2.3957594 \quad 3.5063919$

C $1.3038245 \quad 1.0993401 \quad 2.8285581$

C $1.2538337 \quad 3.29037720 .0000000$

C $1.30096912 .6480995-1.2561766$

$\begin{array}{llll}C & 1.3540991 & 3.3533560 & -2.5345576\end{array}$

C $1.3703333 \quad 2.3957594 \quad-3.5063919$

C $1.3038245 \quad 1.0993401-2.8285581$

N $\quad 1.2943650 \quad 1.2886071 \quad-1.4671499$

Fe $1.0150139-0.15367780 .0000000$

N $\quad 1.1917617 \quad-1.5988836 \quad 1.4765063$

C $0.9444500 \quad-2.9340108 \quad 1.2590711$

$\begin{array}{llll}\text { C } & 0.7743619 & -3.6238896 & 2.5368675\end{array}$

$\begin{array}{llll}\text { C } & 0.8972288 & -2.6764341 & 3.5121234\end{array}$

$\begin{array}{llll}\text { C } & 1.1432516 & -1.4009244 & 2.8369625\end{array}$

$\begin{array}{llll}\text { C } & 1.2143334 & -0.1481195 & -3.4933864\end{array}$

$\begin{array}{llll}\text { C } & 1.1432516 & -1.4009244 & -2.8369625\end{array}$

$\begin{array}{llll}\text { C } & 0.8972288 & -2.6764341 & -3.5121234\end{array}$

$\begin{array}{llll}\text { C } & 0.7743619 & -3.6238896 & -2.5368675\end{array}$

C $0.9444500-2.9340108-1.2590711$

N $1.1917617 \quad-1.5988836-1.4765063$

C $0.8145002 \quad-3.5618696 \quad 0.0000000$

C $1.2143334-0.1481195 \quad 3.4933864$
H $\quad \begin{array}{llll}\text { H } & 1.4144771 & 2.5374227 & -4.5889599\end{array}$

$\begin{array}{lllll}H & 1.3847585 & 4.4393801 & -2.6556625\end{array}$

H $\quad 0.8009093 \quad-2.8089818-4.5927685$

H $\quad 0.5731478-4.6920682 \quad-2.6547639$

$\begin{array}{llll}H & 0.8009093 & -2.8089818 & 4.5927685\end{array}$

H $0.5731478-4.6920682 \quad 2.6547639$

$\begin{array}{llll}\text { H } & 1.3847585 & 4.4393801 & 2.6556625\end{array}$

$\begin{array}{lllll}H & 1.4144771 & 2.5374227 & 4.5889599\end{array}$

C $1.0303758 \quad 4.7673377 \quad 0.0000000$

C $1.1150317-0.1330268 \quad 4.9816936$

C $1.1150317-0.1330268-4.9816936$

C $0.3832337 \quad-4.9933010 \quad 0.0000000$

C $-0.9929922-5.3241503 \quad 0.0000000$

C $-1.4324273-6.6562508 \quad 0.0000000$

C $-0.4855590 \quad-7.6890883 \quad 0.0000000$

$\begin{array}{llll}\text { C } & 0.8864308 & -7.4035246 & 0.0000000\end{array}$

C $1.3017140-6.0642608 \quad 0.0000000$

C $\quad 0.0110755 \quad 0.4822118 \quad-5.6307789$

$\begin{array}{llll}\text { C } & -0.0645966 & 0.5052906 & -7.0333427\end{array}$

C $0.9459182-0.0824140-7.8072992$

C $2.0381725-0.7017693-7.1800199$

$\begin{array}{llll}\text { C } & 2.1170407 & -0.7219288 & -5.7807396\end{array}$

$\begin{array}{llll}\text { C } & 0.0110755 & 0.4822118 & 5.6307789\end{array}$

$\begin{array}{llll}\text { C } & -0.0645966 & 0.5052906 & 7.0333427\end{array}$

C $0.9459182-0.0824140 \quad 7.8072992$
C $2.0381725 \quad-0.7017693 \quad 7.1800199$

$\begin{array}{llll}\text { C } 2.1170407 & -0.7219288 & 5.7807396\end{array}$

C $2.0816352 \quad 5.7084360 \quad 0.0000000$

C $1.8441898 \quad 7.0907154 \quad 0.0000000$

C $\quad 0.5220298 \quad 7.5545028 \quad 0.0000000$

$\begin{array}{llll}\text { C } & -0.5528619 & 6.6551452 & 0.0000000\end{array}$

$\begin{array}{llll}\text { C } & -0.2889361 & 5.2785688 & 0.0000000\end{array}$

Cl $3.0172855 \quad-5.7090295 \quad 0.0000000$

H $\quad 1.6335772 \quad-8.2065594 \quad 0.0000000$

$\begin{array}{llll}H & -0.8224406 & -8.7347495 & 0.0000000\end{array}$

$\begin{array}{llll}\text { Cl } & -2.1759862 & -4.0388395 & 0.0000000\end{array}$

H $-2.5078656-6.87176030 .0000000$

H $2.9787955-1.1866608 \quad 5.2814893$

$\begin{array}{llll}H & -0.9315791 & 0.9941229 & 7.4975288\end{array}$

$\begin{array}{llll}\text { H } & 0.8771870 & -0.0569589 & 8.9038284\end{array}$

H $\quad 2.8355471 \quad-1.1616762 \quad 7.7797201$

$\begin{array}{llll}\mathrm{H} & 2.6899622 & 7.7891529 & 0.0000000\end{array}$

$\begin{array}{llll}\text { Cl } & 3.7343842 & 5.1317792 & 0.0000000\end{array}$

H $\quad 0.3255032 \quad 8.6353095 \quad 0.0000000$

H $\quad-1.5904577 \quad 7.0104594 \quad 0.0000000$

Cl $-1.6321143 \quad 4.1550070 \quad 0.0000000$

$\begin{array}{llll}\text { O } & -0.9688636 & 1.1271133 & -4.9132259\end{array}$

H $\quad-0.9315791 \quad 0.9941229-7.4975288$

H $\quad 0.8771870-0.0569589-8.9038284$

H $\quad 2.8355471 \quad-1.1616762 \quad-7.7797201$ 
H $\quad 2.9787955-1.1866608$-5.2814893

C $\quad-1.9167972 \quad 0.2908587 \quad-4.2247040$

$\begin{array}{llll}\text { C } & -2.8145691 & 1.2109344 & -3.4035946\end{array}$

H $\quad-1.4024553-0.4269742-3.5550951$

$\begin{array}{llll}H & -2.5255370 & -0.2692438 & -4.9658669\end{array}$

H $\quad-2.2528418 \quad 1.6589080-2.5648116$

H $\quad-3.20261892 .0182714 \quad-4.0533580$

\begin{tabular}{llll}
\hline & -3.2026189 & 2.0182714 & -4.0533580
\end{tabular}

C $\quad-1.9167972 \quad 0.2908587 \quad 4.2247040$
H $\quad-1.4024553 \quad-0.4269742 \quad 3.5550951$

H $\quad-2.5255370 \quad-0.2692438 \quad 4.9658669$

C $\quad-2.8145691 \quad 1.2109344 \quad 3.4035946$

H $\quad-2.2528418 \quad 1.6589080 \quad 2.5648116$

H $\quad-3.20261892 .0182714 \quad 4.0533580$

$\begin{array}{llll}\text { O } & -3.9670419 & 0.4801795 & 2.9593425\end{array}$

$\begin{array}{llll}\text { O } & -3.9670419 & 0.4801795 & -2.9593425\end{array}$

$\begin{array}{llll}\text { C }-4.1875613 & -0.0311548 & -1.7022833\end{array}$

$\begin{array}{llll}\text { C } & -4.1875613 & -0.0311548 & 1.7022833\end{array}$ $\begin{array}{llll}0 & -5.2897264 & -0.4855144 & 1.4700018\end{array}$

$\begin{array}{llll}0 & -5.2897264 & -0.4855144 & -1.4700018\end{array}$

$\begin{array}{llll}\text { C } & -3.0756877 & -0.0349018 & -0.7141834\end{array}$

$\begin{array}{llll}C & -3.0756877 & -0.0349018 & 0.7141834\end{array}$

N $-1.0513488-0.15656090 .0000000$

$\begin{array}{llll}N & -1.7815032 & -0.1059040 & 1.1069767\end{array}$

N $-1.7815032-0.1059040-1.1069767$

\section{Compound 7C}

\section{$E_{P B E(D 3) / d e f 2 S V P D}=-5931.981930716$ Hartree}

\section{Nimag $=0$}

C $-2.1418946-2.2215586-1.1466152$

N $-1.8861708-0.8694109-1.2119040$

$\begin{array}{llll}\text { C } & -3.5623075 & -2.4469774 & -0.9296375\end{array}$

C $-4.1491851-1.2118550-0.8451526$

$\begin{array}{llll}\text { C } & -3.0886579 & -0.2314799 & -1.0304457\end{array}$

$\begin{array}{llll}\text { C } & -1.1677348 & -3.2372584 & -1.2210934\end{array}$

C $0.2439962-3.0703133-1.3334173$

$\begin{array}{llll}\text { C } & 1.2033714 & -4.1490181 & -1.3545997\end{array}$

$\begin{array}{llll}C & 2.4544519 & -3.5673986 & -1.2860611\end{array}$

C $2.2429688-2.1401802 \quad-1.2209084$

N $0.8972689-1.8639804-1.2987166$

Fe $-0.0000000 \quad 0.0000000-1.0698618$

$\begin{array}{llll}\text { N } & -0.8972689 & 1.8639804 & -1.2987166\end{array}$

C $\quad-0.2439962 \quad 3.0703133 \quad-1.3334173$

$\begin{array}{lllll}C & -1.2033714 & 4.1490181 & -1.3545997\end{array}$

C $-2.4544519 \quad 3.5673986 \quad-1.2860611$

$\begin{array}{llll}\text { C } & -2.2429688 & 2.1401802 & -1.2209084\end{array}$

C $3.2682888-1.1777963-0.9893330$

$\begin{array}{llll}\text { C } 3.0886579 & 0.2314799 & -1.0304457\end{array}$

C $4.1491851 \quad 1.2118550-0.8451526$

C $3.5623075 \quad 2.4469774-0.9296375$

C $2.1418946 \quad 2.2215586-1.1466152$

N $1.8861708 \quad 0.8694109 \quad-1.2119040$

C $\quad 1.1677348 \quad 3.2372584 \quad-1.2210934$

$\begin{array}{llll}\text { C } & -3.2682888 & 1.1777963 & -0.9893330\end{array}$

H $3.4297642 \quad-4.0616610-1.2734589$

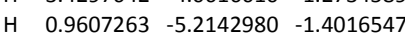

H $\quad \begin{array}{llll}5.2027973 & 0.9787467 & -0.6722035\end{array}$

$\begin{array}{llll}H & 4.0423136 & 3.4256491 & -0.8419723\end{array}$

H $\quad-3.4297642 \quad 4.0616610 \quad-1.2734589$

H $\quad-0.9607263 \quad 5.2142980-1.4016547$

H $\quad-4.0423136-3.4256491-0.8419723$

$\begin{array}{llll}\text { H } & -5.2027973 & -0.9787467 & -0.6722035\end{array}$

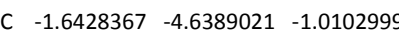

$\begin{array}{llll}\text { C } & -4.6535386 & 1.6806284 & -0.7310731\end{array}$

C $4.6535386-1.6806284-0.7310731$

C $1.6428367 \quad 4.6389021 \quad-1.0102999$

C $1.6827138 \quad 5.1855675 \quad 0.2930506$

$\begin{array}{llll}\text { C } & 2.1198659 & 6.4960143 & 0.5311760\end{array}$

$\begin{array}{llll}\text { C } & 2.5274218 & 7.2868864 & -0.5518423\end{array}$

C $2.5015637 \quad 6.7811696-1.8582660$

C $2.0607977 \quad 5.4671348 \quad-2.0718289$

C $5.1399366 \quad-2.2029898 \quad 0.5104910$

$\begin{array}{llll}\text { C } & 6.4937974 & -2.6124214 & 0.5987875\end{array}$

C $7.3610730-2.5145870-0.4862624$

$\begin{array}{llll}\text { C } & 6.8989942 & -1.9921018 & -1.7080018\end{array}$

C $5.5688264-1.5785695 \quad-1.8071761$

$\begin{array}{llll}\text { C } & -5.1399366 & 2.2029898 & 0.5104910\end{array}$

$\begin{array}{llll}\text { C } & -6.4937974 & 2.6124214 & 0.5987875\end{array}$

$\begin{array}{lllll}\text { C } & -7.3610730 & 2.5145870 & -0.4862624\end{array}$

$\begin{array}{llll}\text { C } & -6.8989942 & 1.9921018 & -1.7080018\end{array}$

C $\quad-5.5688264 \quad 1.5785695 \quad-1.8071761$

$\begin{array}{llll}\text { C } & -2.0607977 & -5.4671348 & -2.0718289\end{array}$

$\begin{array}{llll}\text { C } & -2.5015637 & -6.7811696 & -1.8582660\end{array}$

C $-2.5274218-7.2868864-0.5518423$

$\begin{array}{llll}C & -2.1198659 & -6.4960143 & 0.5311760\end{array}$

C $-1.6827138 \quad-5.1855675 \quad 0.2930506$

Cl $2.0243380 \quad 4.8356293 \quad-3.7039806$

$\begin{array}{llll}H & 2.8196034 & 7.3941027 & -2.7104983\end{array}$

H $2.8718986 \quad 8.3147220 \quad-0.3741013$

$\begin{array}{lllll}\text { Cl } & 1.1842307 & 4.1936400 & 1.6455007\end{array}$

H $\quad 2.1396471 \quad 6.8838037 \quad 1.5569324$

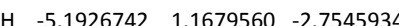

$\begin{array}{llll}\text { O } & -4.4788341 & 2.3062410 & 1.6934478\end{array}$

$\begin{array}{lllll}H & -6.8326384 & 3.0063331 & 1.5660376\end{array}$

$\begin{array}{lllll}H & -8.4034086 & 2.8456965 & -0.3792234\end{array}$
$\begin{array}{llll}H & -7.5663006 & 1.9140234 & -2.5767109\end{array}$

$\begin{array}{llll}H & -2.8196034 & -7.3941027 & -2.7104983\end{array}$

$\begin{array}{lllll}\mathrm{Cl} & -2.0243380 & -4.8356293 & -3.7039806\end{array}$

H $\quad-2.8718986-8.3147220-0.3741013$

H $\quad-2.1396471 \quad-6.8838037 \quad 1.5569324$

$\begin{array}{llll}\text { Cl } & -1.1842307 & -4.1936400 & 1.6455007\end{array}$

$\begin{array}{llll}\text { O } & 4.4788341 & -2.3062410 & 1.6934478\end{array}$

H $\quad 6.8326384-3.00633311 .5660376$

H $8.4034086-2.8456965 \quad-0.3792234$

H $7.5663006-1.9140234-2.5767109$

H $\quad 5.1926742 \quad-1.1679560 \quad-2.7545934$

N $\quad 0.0000000 \quad-0.0000000 \quad 0.9749682$

$\begin{array}{llll}\text { C } & 0.7090864 & -0.0474760 & 3.0042537\end{array}$

$\begin{array}{llll}\text { C } & -0.7090864 & 0.0474760 & 3.0042537\end{array}$

$\begin{array}{llll}\mathrm{N} & -1.1043559 & 0.0404825 & 1.7135207\end{array}$

$\begin{array}{llll}\text { N } & 1.1043559 & -0.0404825 & 1.7135207\end{array}$

C $\quad-1.7355057 \quad 0.0428577 \quad 4.0830057$

C $1.7355057 \quad-0.0428577 \quad 4.0830057$

$\begin{array}{llll}\text { O } & -2.7707311 & 0.9340528 & 3.9511165\end{array}$

O $\quad 2.7707311-0.9340528 \quad 3.9511165$

$\begin{array}{llll}0 & -1.7304102 & -0.7439330 & 5.0080555\end{array}$

$\begin{array}{llll}\text { O } & 1.7304102 & 0.7439330 & 5.0080555\end{array}$

$\begin{array}{llll}\text { C } & 2.6047127 & -2.1791321 & 3.2363575\end{array}$

H $\quad \begin{array}{llll}1.5390283 & -2.4801227 & 3.2564524\end{array}$

H $\quad 3.1844144 \quad-2.9217688 \quad 3.8151138$

$\begin{array}{llll}\text { C } & -2.6047127 & 2.1791321 & 3.2363575\end{array}$

$\begin{array}{llll}H & -1.5390283 & 2.4801227 & 3.2564524\end{array}$

$\begin{array}{llll}H & -3.1844144 & 2.9217688 & 3.8151138\end{array}$

$\begin{array}{llll}\text { C } & -3.0580045 & 2.1799532 & 1.7860356\end{array}$

$\begin{array}{llll}\text { H } & -2.7071054 & 1.2487430 & 1.3031987\end{array}$

H $\quad-2.5778406 \quad 3.0403546 \quad 1.2759457$

C $3.0580045-2.17995321 .7860356$

H $\quad 2.7071054-1.2487430 \quad 1.3031987$

H $2.5778406-3.0403546 \quad 1.2759457$ 


\section{Magnetic Susceptibility - Evans Measurements}

The paramagnetic susceptibility of high-spin and low-spin complexes were determined via the standard Evans measurements using ${ }^{1} \mathrm{H}$ NMR spectroscopy. An NMR tube with a coaxial insert, both sealable, was used. For the high-spin complex 17c the outer tube was filled with a $767 \mu \mathrm{M}$ solution of strapped triazole porphyrins $17 \mathrm{c}$ in acetone- $\mathrm{d}_{6}(530 \mu \mathrm{L})$ and the inner tube was filled with a $767 \mu \mathrm{M}$ solution of diamagnetic strapped zinc porphyrin in acetone- $d_{6}(60 \mu \mathrm{L})$. The strapped zinc porphyrin is completely diamagnetic and compensates the diamagnetic contribution of the porphyrin macrocycle to the observed magnetic susceptibility. For the deprotonated, neutral strapped high-spin complex 18, the outer tube was filled with a $767 \mu \mathrm{M}$ solution of strapped porhyrin 18 in acetone- $\mathrm{d}_{6}(530 \mu \mathrm{L})$ and the inner tube was filled with a $767 \mu \mathrm{M}$ solution of diamagnetic strapped zinc porphyrin in acetone$\mathrm{d}_{6}(60 \mu \mathrm{L})$. For the strapped low-spin complex 20, the outer tube was filled with a 767 $\mu \mathrm{M}$ solution of strapped porphyrin 18 containing 100 eq. p-methoxypyridine in acetone- $\mathrm{d}_{6}(530 \mu \mathrm{L})$ and the inner tube was filled with a $767 \mu \mathrm{M}$ solution of diamagnetic strapped zinc porphyrin containing 100 eq. $p$-methoxypyridine in acetone- $\mathrm{d}_{6}(60 \mu \mathrm{L})$. For the strapped low-spin complex 21, the outer tube was filled with a $767 \mu \mathrm{M}$ solution of strapped porphyrin 18 containing 50 eq. trans-4-chloro-3(3,5-ditert-butylphenyl)azo-pyridine 22 in acetone- $\mathrm{d}_{6}(530 \mu \mathrm{L})$ and the inner tube was filled with a $767 \mu \mathrm{M}$ solution of diamagnetic strapped zinc porphyrin containing $50 \mathrm{eq}$. trans-4-chloro-3-(3,5-ditert-butylphenyl)azo-pyridine 22 in acetone- $\mathrm{d}_{6}(60 \mu \mathrm{L})$. For the strapped low-spin complex 18 with cis-4-chloro-3-(3,5-ditert-butylphenyl)azo-pyridine 22, the outer tube was filled with a $767 \mu \mathrm{M}$ solution of strapped porphyrin 18 containing 50 eq. cis-4-chloro-3-(3,5-ditert-butylphenyl)azo-pyridine 22 in acetone- $\mathrm{d}_{6}$ (530 $\mu \mathrm{L}$ ) and the inner tube was filled with a $767 \mu \mathrm{M}$ solution of diamagnetic strapped zinc porphyrin containing 50 eq. cis-4-chloro-3-(3,5-ditert-butylphenyl)azo-pyridine 22 in acetone- $\mathrm{d}_{6}(60 \mu \mathrm{L})$. The concentration of the internal standard, TMS (tetramethylsilane), was maintained constant in the inner and outer tubes. ${ }^{1} \mathrm{H}$ NMR spectra were recorded in a $600 \mathrm{MHz}$ spectrometer at a constant temperature of 300 $\mathrm{K}$. The sample (inner and outer tubes) was allowed to equilibrate at this temperature for at least 15 minutes before measurement. The following supplementary equation (1) was used to calculate the paramagnetic susceptibility from the experimentally measured shift in TMS signals between the inner and outer tubes. 


$$
\chi_{M}=\frac{3 \delta f M}{4 \pi f m}+\chi_{M}^{o}+\frac{\chi_{M}^{o}\left(d_{0}-d_{x}\right)}{m}-\chi_{d i a}^{M}
$$

where,

$X_{M}=$ Molar paramagnetic susceptibility in $\left(\mathrm{cm}^{3} \mathrm{~mol}^{-1}\right)$

$\delta f=$ Frequency difference between the TMS peaks of the inner and outer tube $(\mathrm{Hz})$

$M=$ Molecular weight of the substance $\left(\mathrm{gmol}^{-1}\right)$

$f=$ Frequency of the NMR instrument $(\mathrm{Hz})$

$m=$ Mass of the substance in $1 \mathrm{~mL}$ of solution $(\mathrm{g})$

$X_{M}{ }_{M}=$ Mass susceptibility of the solvent $\left(\mathrm{cm}^{3} \mathrm{~mol}^{-1}\right)$

$d_{0}=$ Density of the solvent $\left(\mathrm{g} \mathrm{cm}^{-3}\right)$

$d_{x}=$ Density of the solution $\left(\mathrm{g} \mathrm{cm}^{-3}\right)$

$X^{M}{ }_{\text {dia }}=$ Diamagnetic correction to the magnetic susceptibility $\left(\mathrm{cm}^{3} \mathrm{~mol}^{-1}\right)$

Since all the solutions used in this experiment are dilute, the density of the solvent and the solutions may be considered equal, thereby nullifying the factor $\left(d_{0}-d_{x}\right)$. The diamagnetic correction ( $X^{\mathrm{M}}{ }_{\text {dia }}$ ) has been taken care of by using equivalent diamagnetic components in the inner tube. Since the same solvents are used in the inner and outer tubes, the solvent correction factor $x^{0}$ could also be neglected. Thus the equation for calculating the molar paramagnetic susceptibility effectively reduces to Supplementary Equation (2).

$$
\mathrm{X}_{\mathrm{M}}=\frac{3 \delta f M}{4 \pi f m}
$$

From the known values of molar paramagnetic susceptibility, the corresponding magnetic moment $\left(\mu_{\text {eff }}\right)$ may be obtained via Supplementary Equation (3).

$$
\mu_{\text {eff }}=2.828 \sqrt{\chi_{M} T}
$$

where $\mathrm{T}=$ Temperature $(\mathrm{K})$ 
For a $767 \mu \mathrm{M}$ solution of the 5,15 -strapped iron(III) porphyrin chloride $17 \mathrm{c}$,

$$
\begin{aligned}
& \delta f=12.5 \mathrm{~Hz} \\
& X_{\mathrm{M}}=0.01468 \mathrm{~cm}^{3} \mathrm{~mol}^{-1} \\
& \mu_{\text {eff }}=5.92 \text { B.M. }
\end{aligned}
$$

For a $767 \mu \mathrm{M}$ solution of the neutral 5,15-strapped iron(III) porphyrin $\mathbf{1 8}$,

$$
\begin{aligned}
& \delta f=11.7 \mathrm{~Hz} \\
& \chi_{\mathrm{M}}=0.01325 \mathrm{~cm}^{3} \mathrm{~mol}^{-1} \\
& \mu_{\text {eff }}=5.62 \text { B.M. }
\end{aligned}
$$

For a $767 \mu \mathrm{M}$ solution of the neutral 5,15-strapped iron(III) porphyrin with p-methoxypyridine $\mathbf{2 0}$,

$$
\begin{aligned}
& \delta f=2.50 \mathrm{~Hz} \\
& X_{\mathrm{M}}=0.00288 \mathrm{~cm}^{3} \mathrm{~mol}^{-1} \\
& \mu_{\text {eff }}=2.56 \text { B.M. }
\end{aligned}
$$

For a $767 \mu \mathrm{M}$ solution of the neutral 5,15-strapped iron(III) porphyrin 18 with trans-4chloro-3-(3,5-ditert-butylphenyl)azo-pyridine 22,

$$
\begin{aligned}
& \delta f=4.20 \mathrm{~Hz} \\
& X_{\mathrm{M}}=0.00476 \mathrm{~cm}^{3} \mathrm{~mol}^{-1} \\
& \mu_{\text {eff }}=3.44 \text { B.M. }
\end{aligned}
$$

For a $767 \mu \mathrm{M}$ solution of the neutral 5,15-strapped iron(III) porphyrin 18 with cis-4chloro-3-(3,5-ditert-butylphenyl)azo-pyridine 22,

$$
\begin{aligned}
& \delta f=6.81 \mathrm{~Hz} \\
& X_{\mathrm{M}}=0.00771 \mathrm{~cm}^{3} \mathrm{~mol}^{-1} \\
& \mu_{\text {eff }}=4.30 \text { B.M. }
\end{aligned}
$$




\section{Experimental data of the spin switch}

\section{V.1 Experimental data with FeTPPCI 1 and DMTD-}

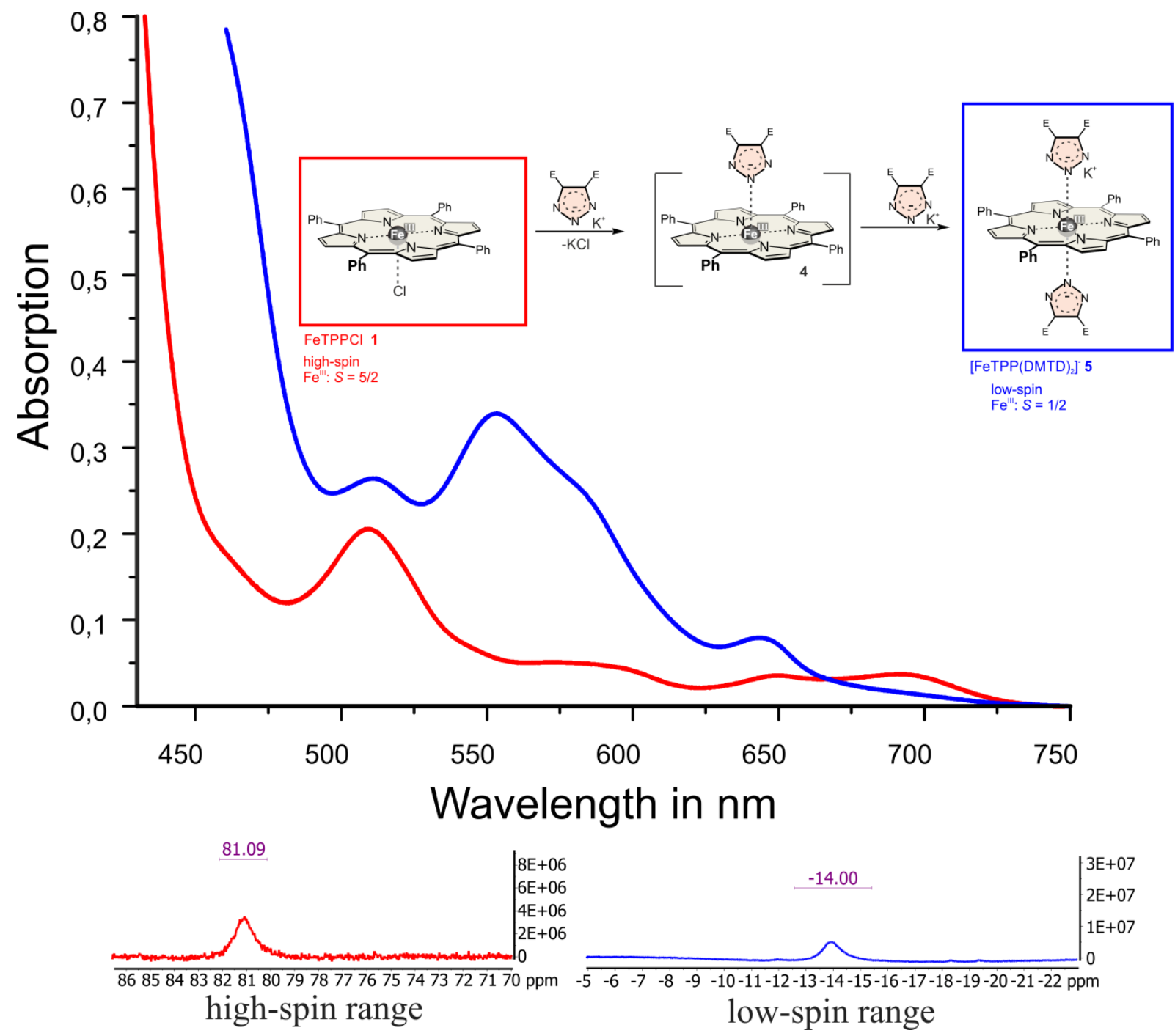

Figure S2: UV spectra of FeTPPCI $1\left(50 \mu \mathrm{M}\right.$, acetonitrile, $\left.25^{\circ} \mathrm{C}\right)$ before (red) and after addition of 1000 eq. DMTD- (blue) and the high-spin and low-spin range in the ${ }^{1} \mathrm{H}$ NMR (500 MHz (Avance Neo), acetonitrile-d $3,300 \mathrm{~K}$ ). 


\section{V.2 Experimental data with $17 \mathrm{c}$ and $\mathrm{K}_{2} \mathrm{CO}_{3}$}

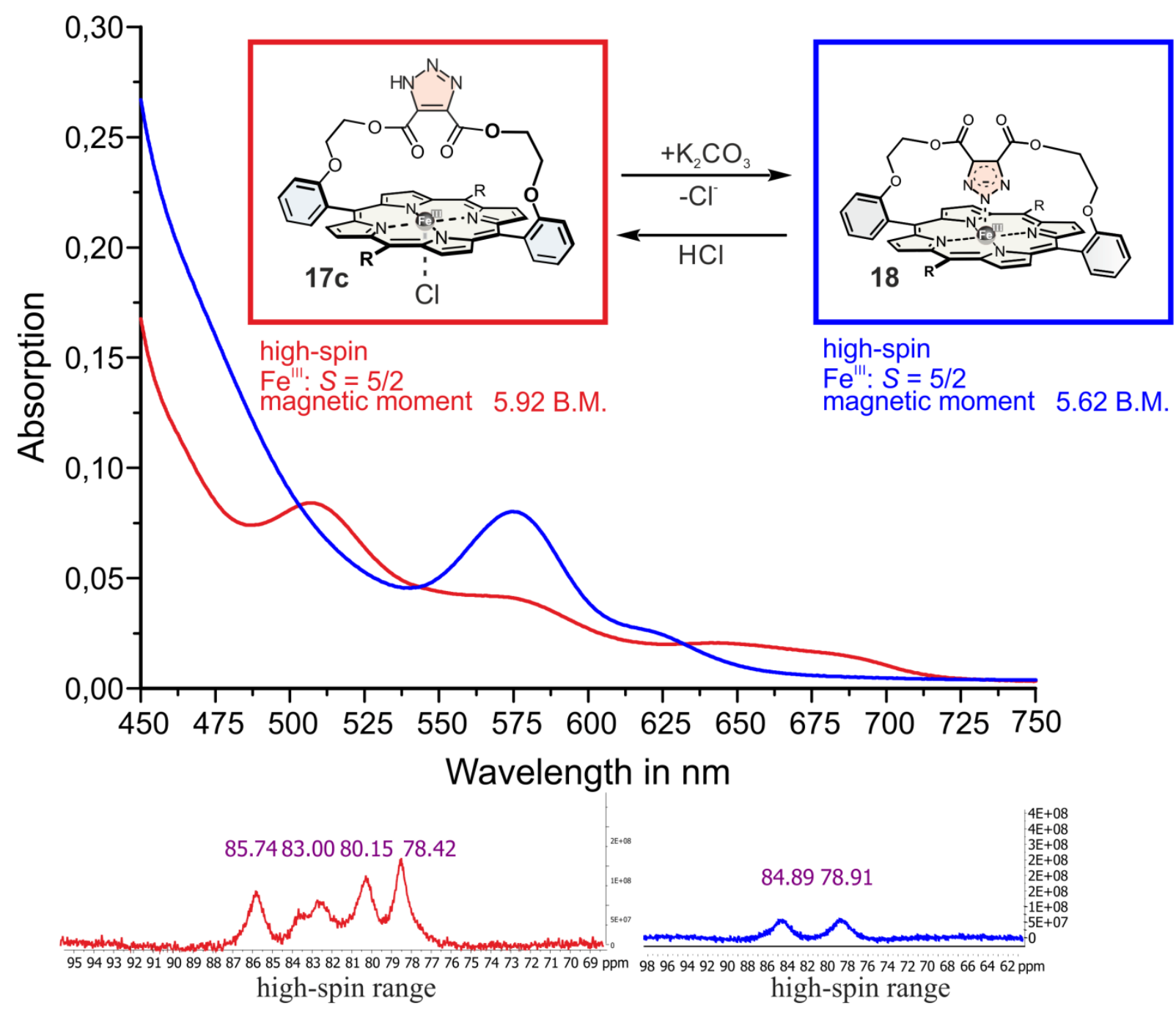

Figure S3: UV spectra of $17 \mathrm{c}\left(10 \mu \mathrm{M}\right.$, acetonitrile, $\left.25^{\circ} \mathrm{C}\right)$ before (red) and after addition of $\mathrm{K}_{2} \mathrm{CO}_{3}$ (blue) and the high-spin range in the ${ }^{1} \mathrm{H}$ NMR (500 MHz (Avance Neo), acetonitrile- $d_{3}, 300 \mathrm{~K}$ ).

High resolution mass spectrometry of 18.

MS: (HR) ESI

\begin{tabular}{|l|l|l|l|}
\hline calc. & found & Mass. Diff (ppm) & Molecular formula \\
\hline 1043.04806 & 1043.04788 & -0.17 & $\mathrm{C}_{52} \mathrm{H}_{31} \mathrm{Cl}_{4} \mathrm{FeN}_{7} \mathrm{O}_{6}$ \\
\hline
\end{tabular}




\section{V.3 Experimental data with 18 and DMTD-}

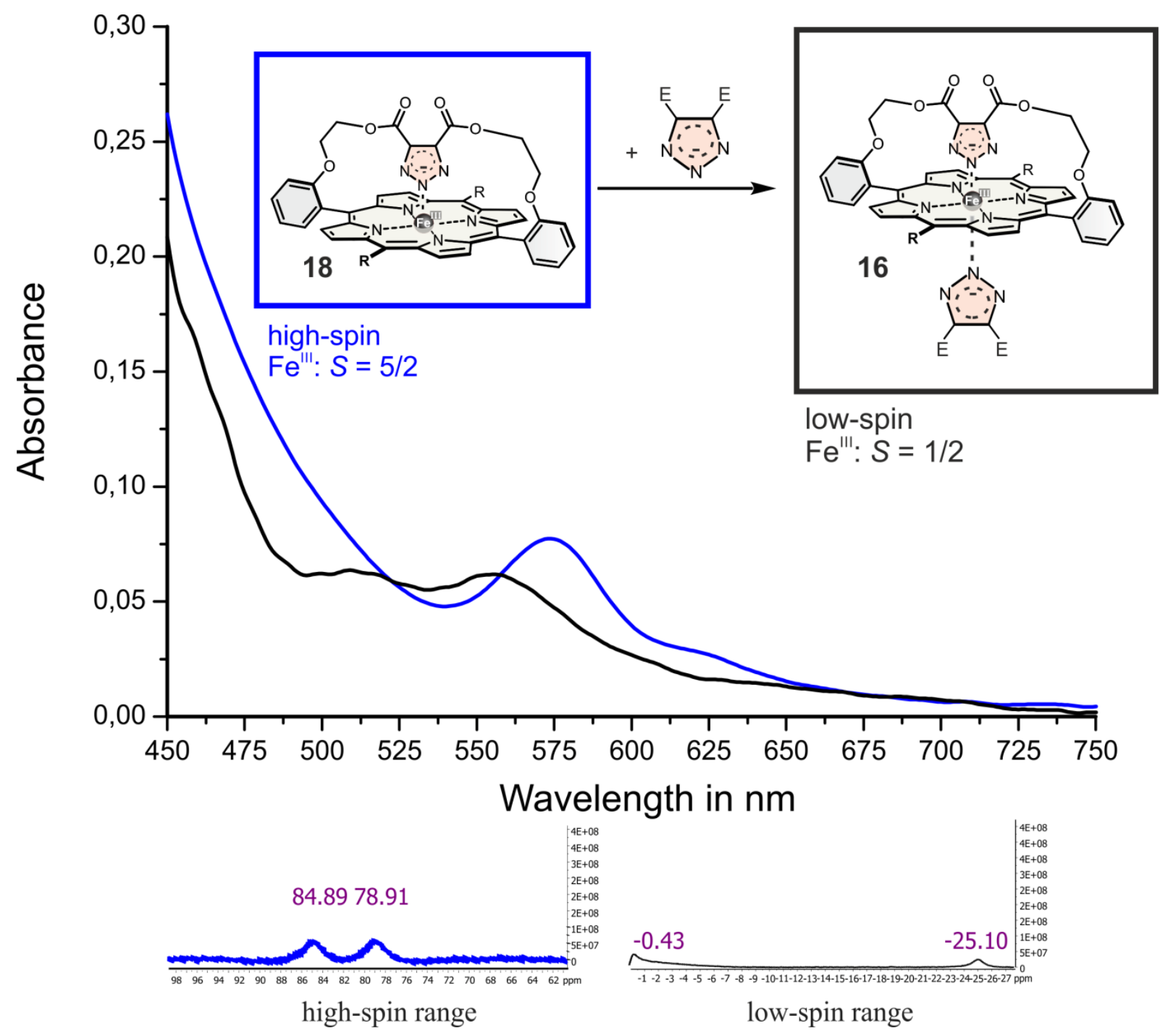

Figure S4: UV spectra of $18\left(10 \mu \mathrm{M}\right.$, acetonitrile, $\left.25^{\circ} \mathrm{C}\right)$ before (blue) and after addition of 250 eq. DMDT $^{-}$(black) and the high-spin and low-spin range in the ${ }^{1} \mathrm{H}$ NMR (500 MHz (Avance Neo), acetonitrile-d $3,300 \mathrm{~K}$ ).

High resolution mass spectrometry of 16 was not possible. 


\section{V.4 Experimental data with 18 and $p$-Methoxypyridine}

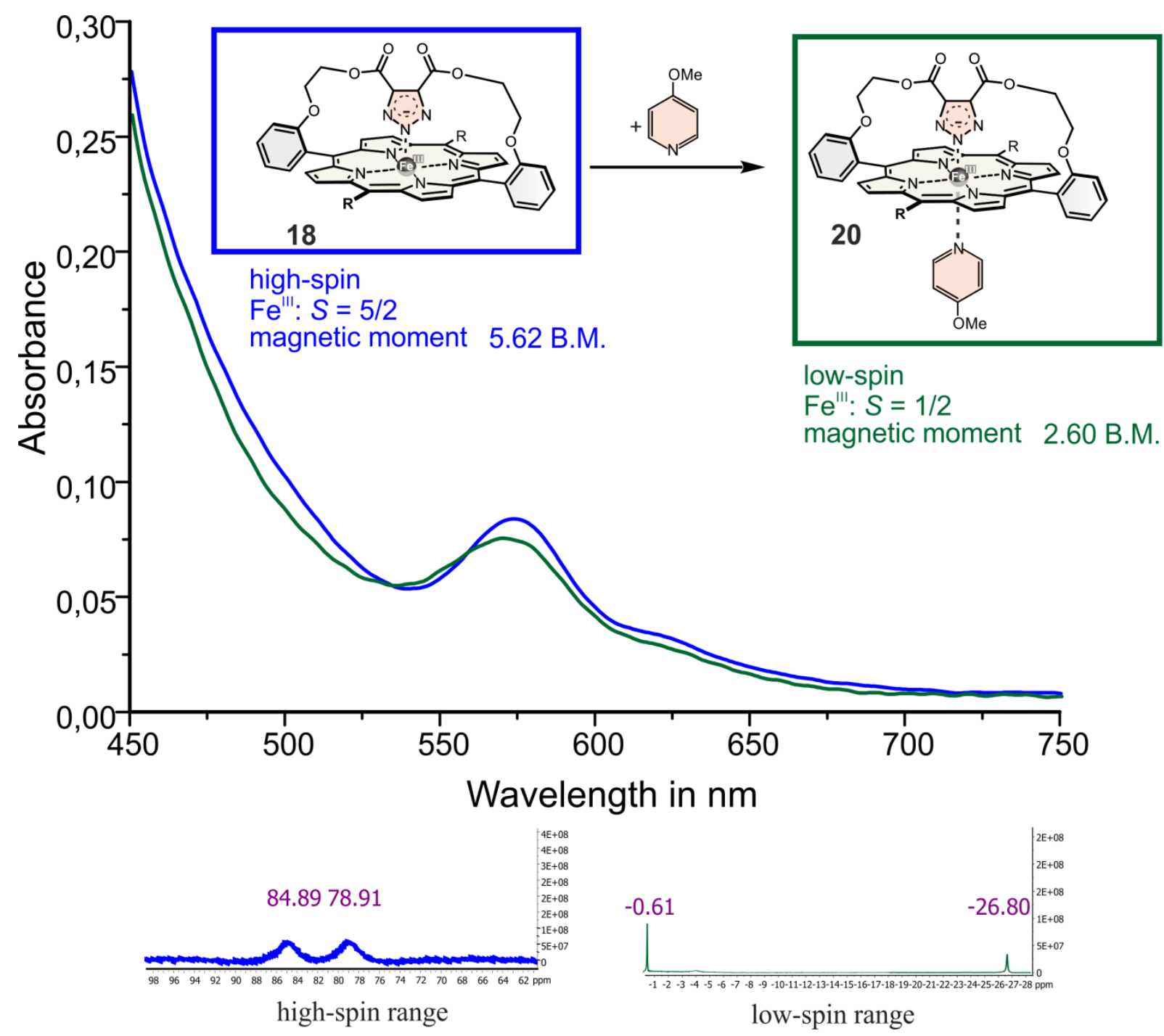

Figure S5: UV spectra of $18\left(10 \mu \mathrm{M}\right.$, acetonitrile, $\left.25^{\circ} \mathrm{C}\right)$ before (blue) and after addition of 100 eq. $p$-Methoxypyridine (green) and the high-spin and low-spin range in the ${ }^{1} \mathrm{H}$ NMR (500 MHz (Avance Neo), acetonitrile- $\mathrm{d}_{3}, 300 \mathrm{~K}$ ).

High resolution mass spectrometry of 20 .

MS: (HR) ESI

\begin{tabular}{|l|l|l|l|}
\hline calc. & found & Mass. Diff $(\mathrm{ppm})$ & Molecular formula \\
\hline 1152.10082 & 1152.10115 & 0.28 & $\mathrm{C}_{58} \mathrm{H}_{38} \mathrm{O}_{7} \mathrm{~N}_{8} \mathrm{Cl}_{4} \mathrm{Fe}$ \\
\hline
\end{tabular}


V.5 Experimental data with 18 and 4-chloro-3-(3,5-ditert-butylphenyl)azopyridine 22

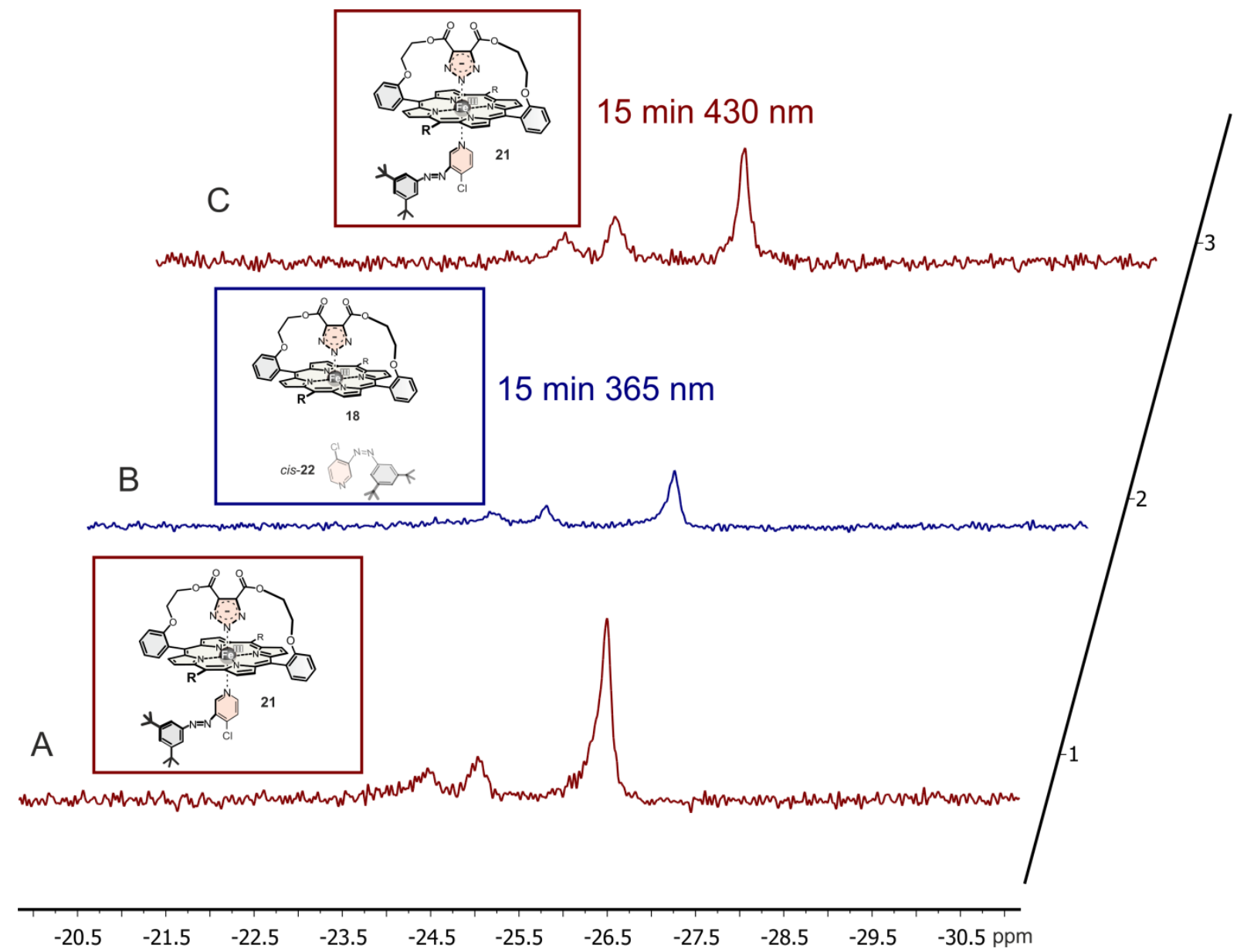

Figure S6: Spin state switching of the strapped ferrous porphyrin 21 in the presence of the photodissociable ligand 22 in the ${ }^{1} \mathrm{H}$ NMR $\left(600 \mathrm{MHz}\right.$, aceton- $\left.\mathrm{d}_{6}, 300 \mathrm{~K}\right)$. 


\section{Data of the crystal structure}

\section{VI.1 Crystal structure of $2^{10}, 2^{20}$-bis(2,6-dichlorophenyl)-4,7,12,15-tetraoxa- 2(5,15)-porpyhrina-1,3(1,2)-dibenzena-cycloheptadecaphane-9-yn-8,11-dione (15c)}

Data collection was performed at $200 \mathrm{~K}$ using an Imaging Plate Diffraction System (IPDS-2) from STOE using Mo-Ka radiation $(\lambda=0.71073 \AA)$. The data processing was performed using $X$-Area ${ }^{6}$ program. The structure was solved by direct methods with SHELXS- $97^{7}$ and structure refinement was performed by full-matrix least squares calculations based on $\mathrm{F}^{8}$ using SHELXL2014 ${ }^{8}$. The hydrogen atoms were positioned with idealized geometry and refined isotopically with $U_{\text {iso }}(H)=1.2 U_{\text {eq }}(C)$ a riding model. After structure refinement several residual electron density peaks were observed in the difference that originate from disordered dichloromethane molecules. Because no reasonable structure model was found the data were corrected for disordered solvent using SQUEEZE in PLATON ${ }^{9}$. Several crystals were tested and all of them were non-merohedrally twinned. If the twinnig was not considered very high reliability factors (wR2 > 40\%; R1 > 14\%) were obtained. Therefore, both individuals were indexed separately and the twin matrix was calculated. Afterwards the data were transformed into the HKLF-5 format using either the option offered by X-Area ${ }^{6}$, the program HKLF-5 written M. Bolte ${ }^{10}$ or by Platon. ${ }^{9}$ Even if the distance that determine if two reflections are overlapped in reciprocal space was optimized, the HKLF-5 data were of poor quality and only a small improvement of the reliability factors were obtained. Therefore, for the final refinement the overlapping reflections were removed during data integration. In this case no complete data were but acceptable reliability factors a flat difference map were obtained and all nonhydrogen atoms can be refined anisotropic with reasonable components of the anisotropic displacement parameters.. It is noted that the asymmetric unit consists of three crystallographically independent molecules and that PLATON suggests a pseudo-translation. However, the pseudo-translation was carefully analysed but it was found that the lattice parameters are correct and not accidently influenced by the twinning.

Crystal data for 15ce: $M=1897.20 \mathrm{~g} / \mathrm{mol}$., block, $0.07 \times 0.13 \times 0.2 \mathrm{~mm}^{3}$, triclinic, space group $P-1, a=14.5805(5) \AA, b=19.1019(8) \AA, c=28.6485(10) \AA, \alpha=$ $100.389(3)^{\circ}, \beta=101.157(3)^{\circ}, \quad Y=108.422(3)^{\circ}, V=7172.8(5) \AA^{3}, Z=6, D_{c}=1.318$ $\mathrm{g} / \mathrm{cm}^{3}, \mathrm{~F} 000=2916, \mu=0.301 \mathrm{~mm}^{-1}, \mathrm{~T}=200 .(1) \mathrm{K}, 2 \theta_{\max }=50.4^{\circ}, 37887$ measured reflections, 16055 independent reflections and 10379 reflections with $\left[I_{\circ}>2 \sigma\left(I_{0}\right)\right]$, $R_{\text {int }}$ $=0.0946$, parameters $=1783, \mathrm{GoF}=1.031, \mathrm{R}=0.0742$ and $w R=0.1887\left[\mathrm{I}_{\circ}>2 \sigma\left(\mathrm{I}_{\circ}\right)\right]$, $\mathrm{R}=0.1130$ and $w R=0.2136$ (all reflections), $0.260<\Delta \rho<-0.243 \mathrm{e} / \AA^{3}$.

CCDC-1885475 (15c) contains the supplementary crystallographic data for this paper. These data can be obtained free of charge from the Cambridge Crystallographic Data Centre via www.ccdc.cam.ac.uk/data request/cif. 


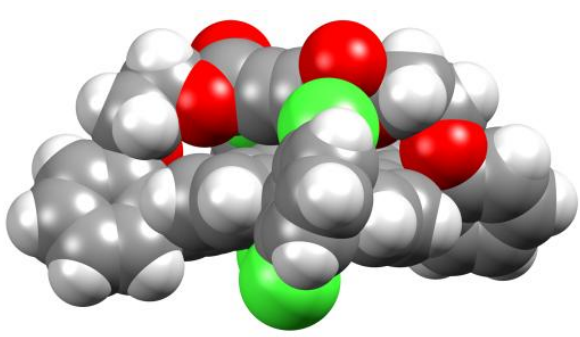

$\mathbf{A}$

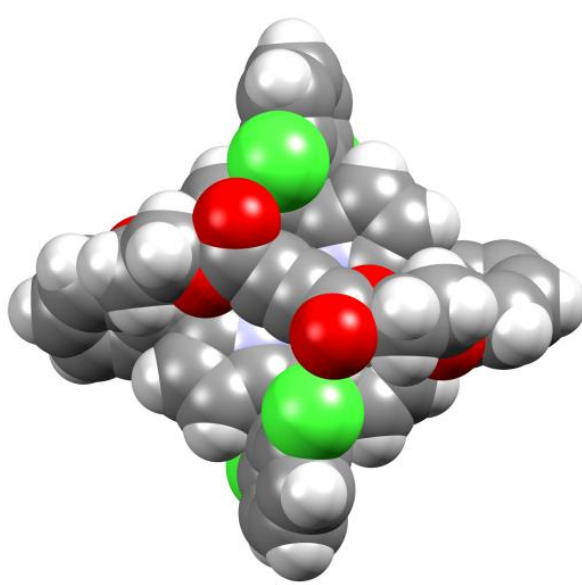

B

Figure S7: The VDW plots of the strapped porphyrin 15c, from side (A) and on top (B). 
VI.2 Single crystal X-ray diffraction study for $2^{10}, 2^{20}$-bis(2,6-dichlorophenyl)-

\section{4,8,12,16-tetraoxa-2(5,15)-porpyhrina-10(4,5)-1,2,3-triazola-1,3(1,2)-dibenzena- cyclohexadecaphane-9,11-dione (16e)}

The diffraction data for $16 \mathrm{e}$ were collected at $120 \mathrm{~K}$ on an Rigaku Oxford diffractometer equipped with an Atlas CCD detector using mirror-monochromatized Cu-Ka radiation $(\lambda=1.5418 \AA)$. The data collection and processing were performed using CrysAlisPro ${ }^{11}$ program. The intensities were corrected with gaussian correction method $^{11}$. The structure was solved by direct methods with the SHELXS- $97^{7}$ program. The structure was refined by full-matrix least squares calculations based on $\mathrm{F}^{8}$ using SHELXL2014 ${ }^{7}$ module incorporated into the OLEX2 ${ }^{12}$ program package. The hydrogen at the triazole unit was located from the $\Delta \mathrm{F}$ map, yet it and all other hydrogen atoms were included in their calculated positions using the 'riding model' in the final refinements (HFIX 431.00 for the triazole hydrogen H2). The SQUEEZE module of PLATON $^{9}$ was utilized in the structure refinement to remove the residual electron densities (43 electrons) from co-crystallized solvent locations, which were not possible to be reliably assign and refine.

Crystal data for $16 \mathrm{e}: \mathrm{M}=1123 \mathrm{~g} / \mathrm{mol}$, needle, $0.048 \times 0.087 \times 0.246 \mathrm{~mm}^{3}$, triclinic, space group $P-1, a=12.9397(8) \AA, b=14.9171(9) \AA, c=15.2735(11) \AA, \alpha=$ $85.457(5)^{\circ}, \beta=89.329(5)^{\circ}, \quad Y=74.627(5)^{\circ}, V=2822.6(3) \AA^{3}, Z=2, D_{c}=1.317$ $\mathrm{g} / \mathrm{cm}^{3}, \mathrm{~F} 000=1168, \mu=2.380 \mathrm{~mm}^{-1}, \mathrm{~T}=120.0(1) \mathrm{K}, 2 \theta_{\max }=66.74^{\circ}$, measured reflections are missing. 9942 independent reflections and 6699 reflections with $\left[\mathrm{I}_{0}>\right.$ $\left.2 \sigma\left(I_{0}\right)\right], R_{\text {int }}=0.0470$, reflections $/$ parameters $/$ restraints $=9942 / 711 / 1, \mathrm{GoF}=1.017, \mathrm{R}$ $=0.0679$ and $w R=0.1767\left[I_{o}>2 \sigma\left(I_{o}\right)\right], R=0.1005$ and $w R=0.2038$ (all reflections), $0.758<\Delta \rho<-0.448 \mathrm{e} / \AA^{3}$.

CCDC-1884544 (16e) contains the supplementary crystallographic data for this paper. These data can be obtained free of charge from the Cambridge Crystallographic Data Centre via www.ccdc.cam.ac.uk/data request/cif. 


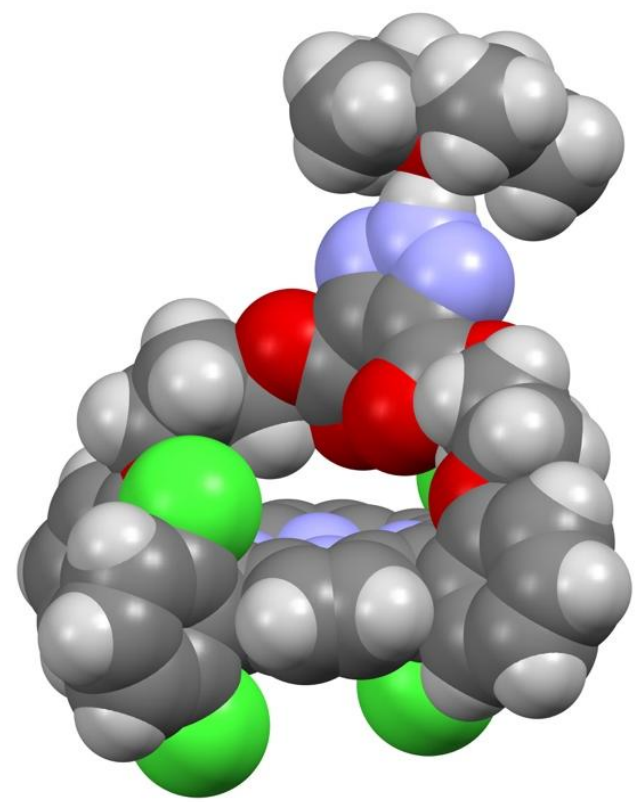

A

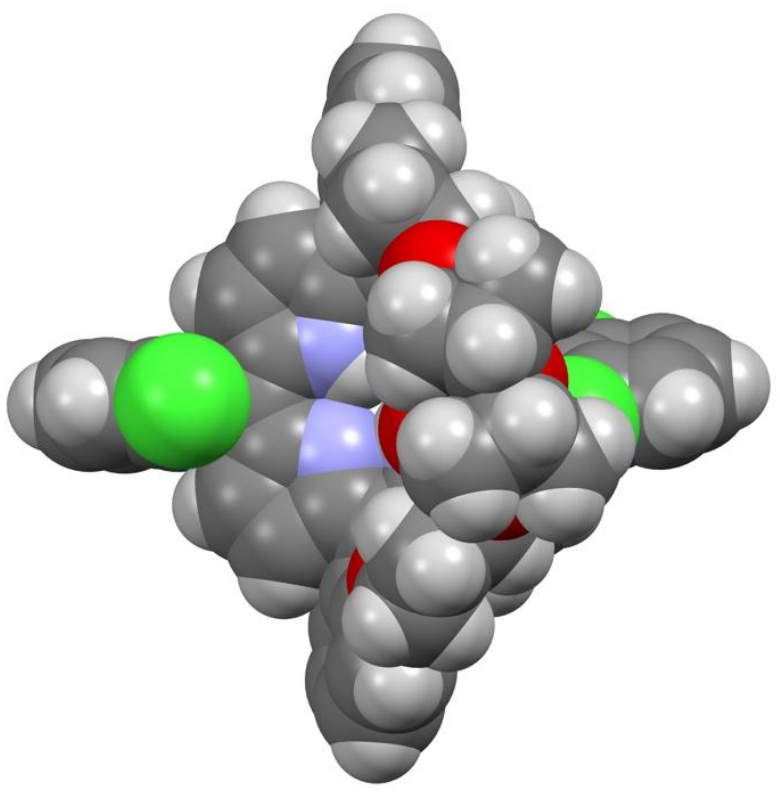

B

Figure S8: The VDW plots of the strapped porphyrin $16 \mathrm{e}$, from side (A) and on top (B).

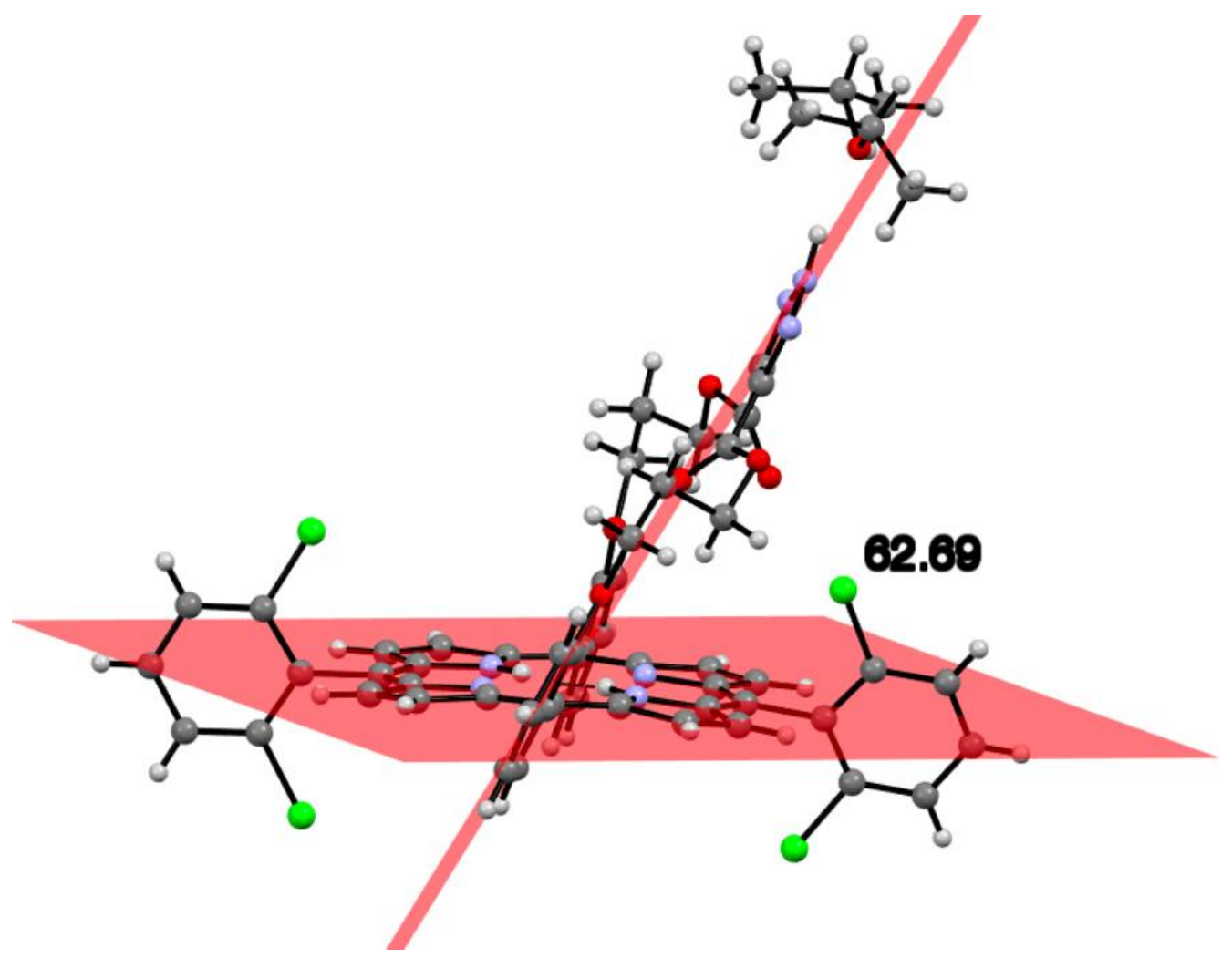

Figure S9: The tilt angle between the strap and the porphyrin plane. 


\section{Photostationary state of trans-4-chloro-3-(3,5-ditert- butylphenyl)azopyridine (22)}

The Photostationary state at $365 \mathrm{~nm}$ : (PSS=8\% trans, $92 \%$ cis $)$

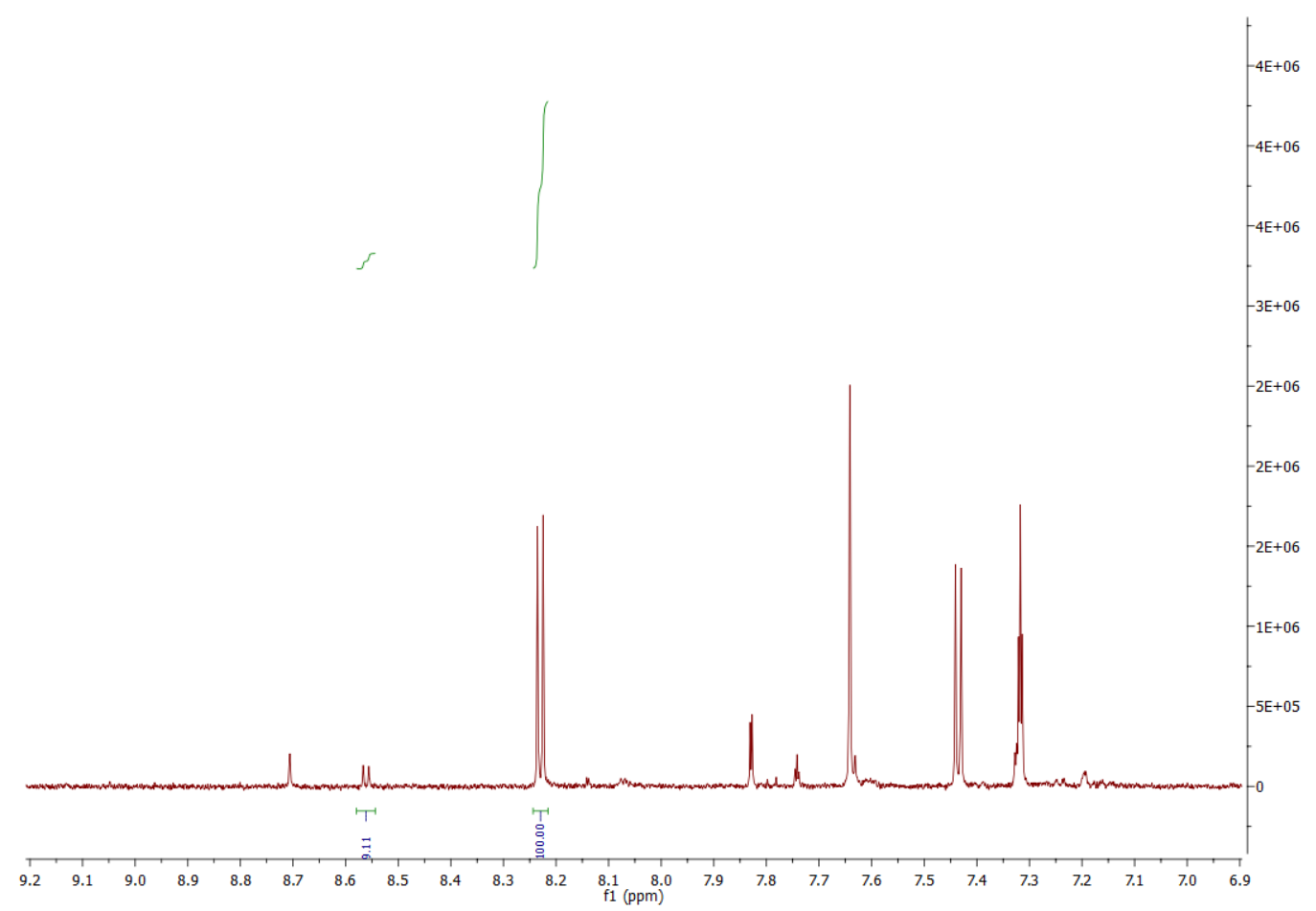

Figure S10: ${ }^{1} \mathrm{H}$ NMR spectrum of the photostationary state of compound 22 at $365 \mathrm{~nm}$ and integration of the trans and cis species in Acetonitrile- $\mathrm{d}_{3}$ at $300 \mathrm{~K}$. 
The Photostationary state at $430 \mathrm{~nm}$ : (PSS=83\% trans, $17 \%$ cis $)$

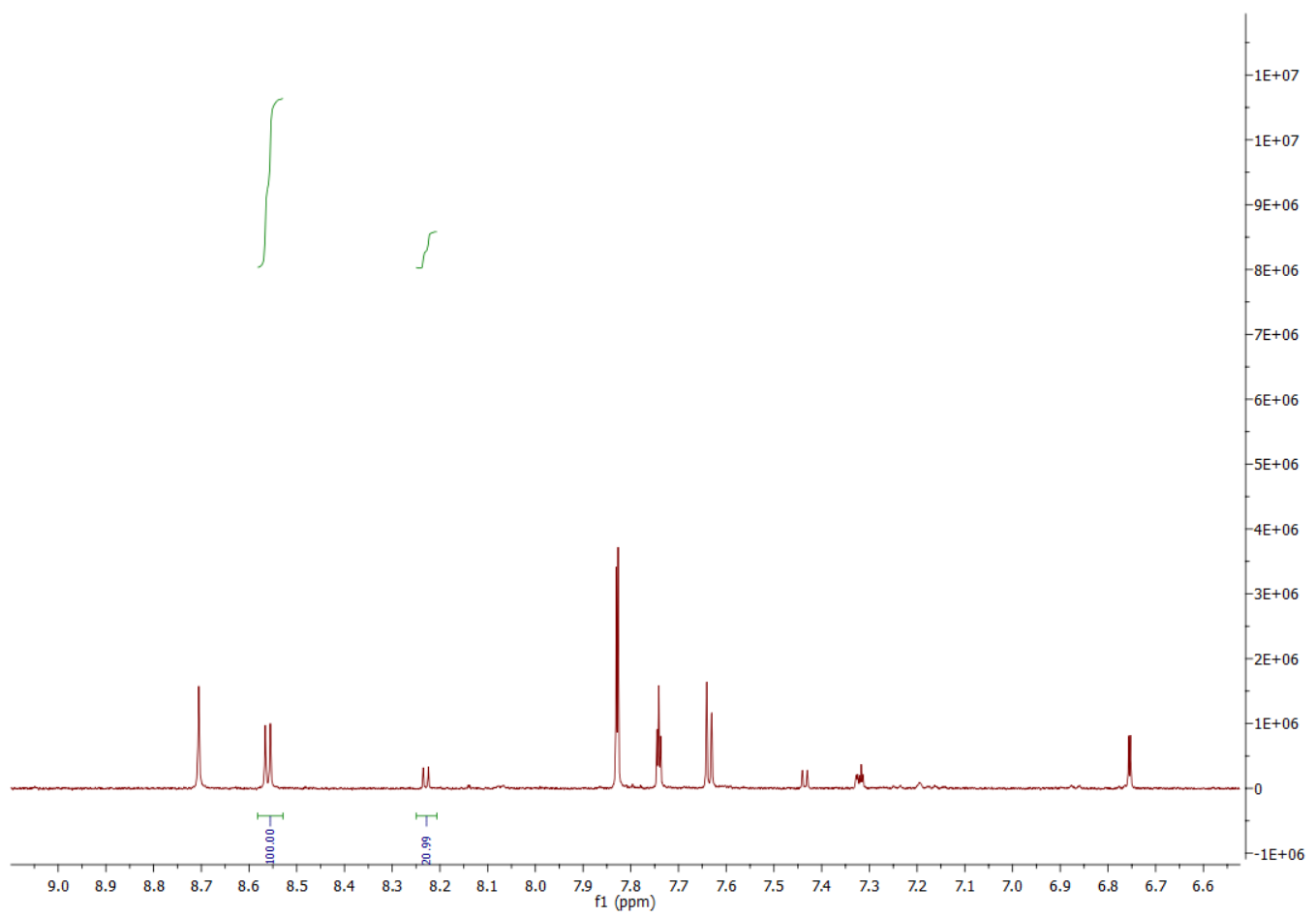

Figure S11: ${ }^{1} \mathrm{H}$ NMR spectrum of the photostationary state of compound 22 at $430 \mathrm{~nm}$ and integration of the trans and cis species in Acetonitrile- $\mathrm{d}_{3}$ at $300 \mathrm{~K}$. 
$\mathrm{S} 40$

VIII. NMR spectra

VIII.1 Bis(2-(2-formylphenoxy)ethyl)but-2-ynedioate (13a)
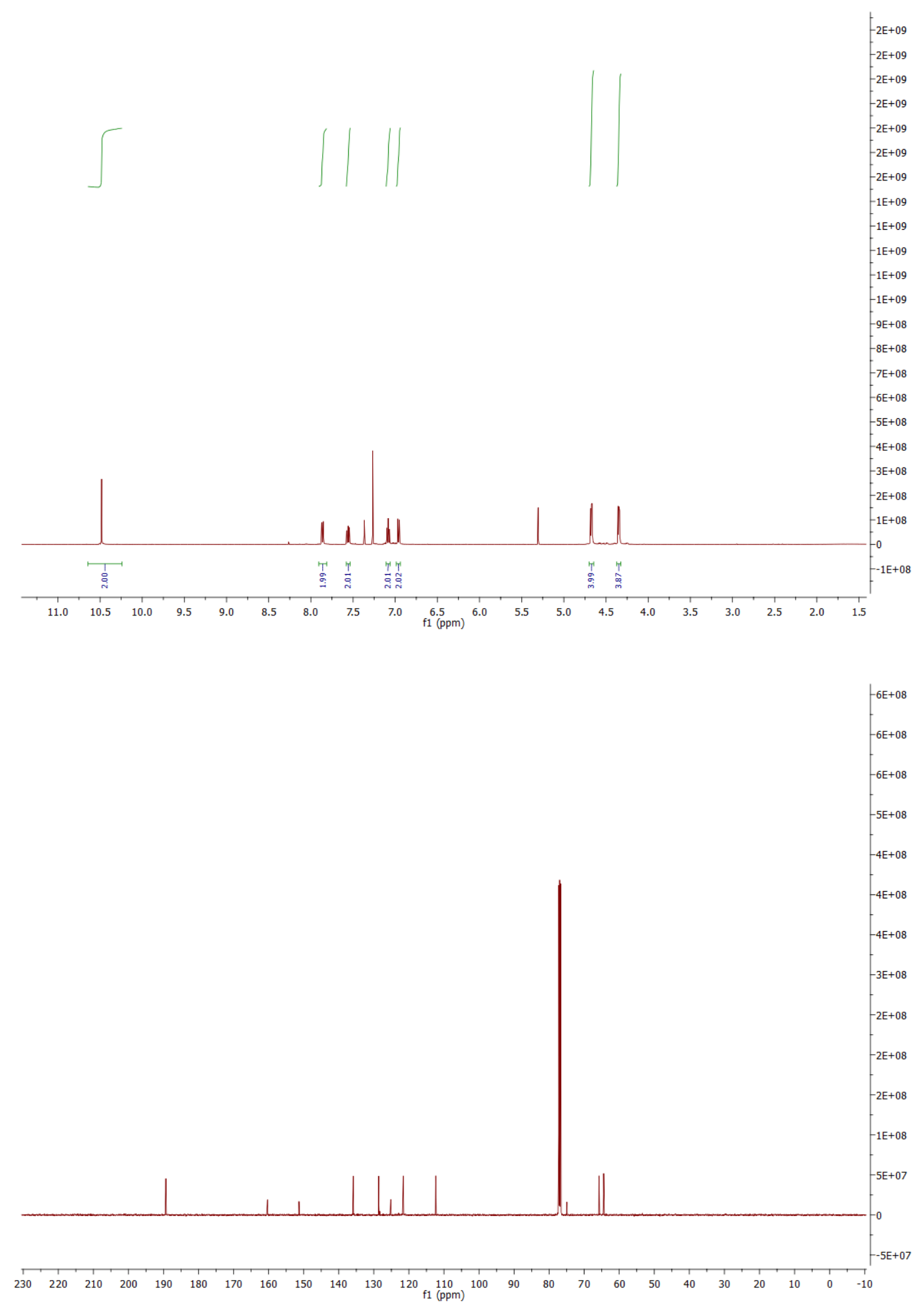
VIII.2 Bis(3-(2-formylphenoxy)propyl)but-2-ynedioate (13b)
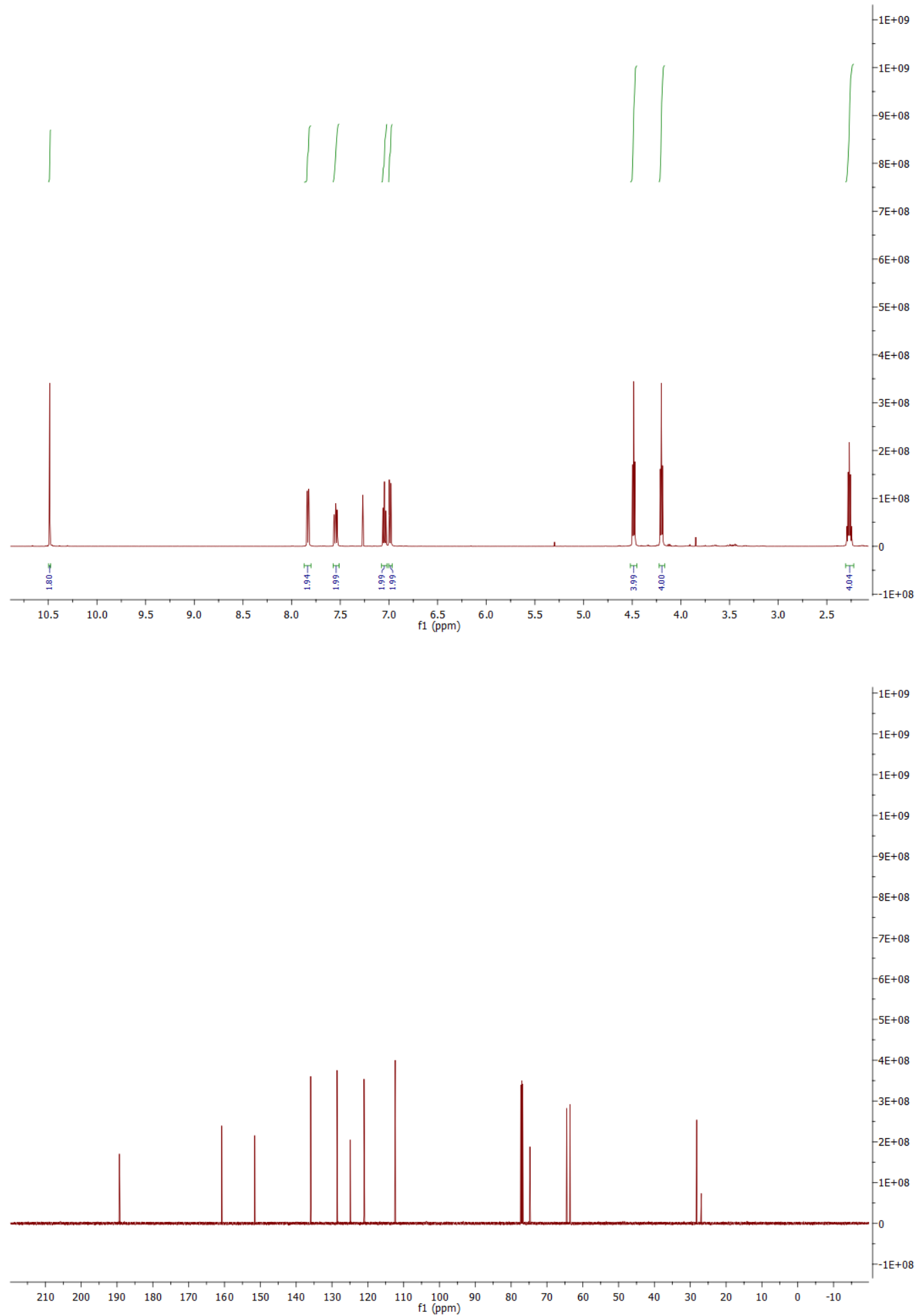
VIII.3 Synthesis of $2^{10}, 2^{20}$-bis(2,3,4,5,6-pentafluorophenyl)-4,7,12,15-tetraoxa2(5,15)-porpyhrina-1,3(1,2)-dibenzena-cyclopentadecaphane-9-yn-8,11-dione (15d)
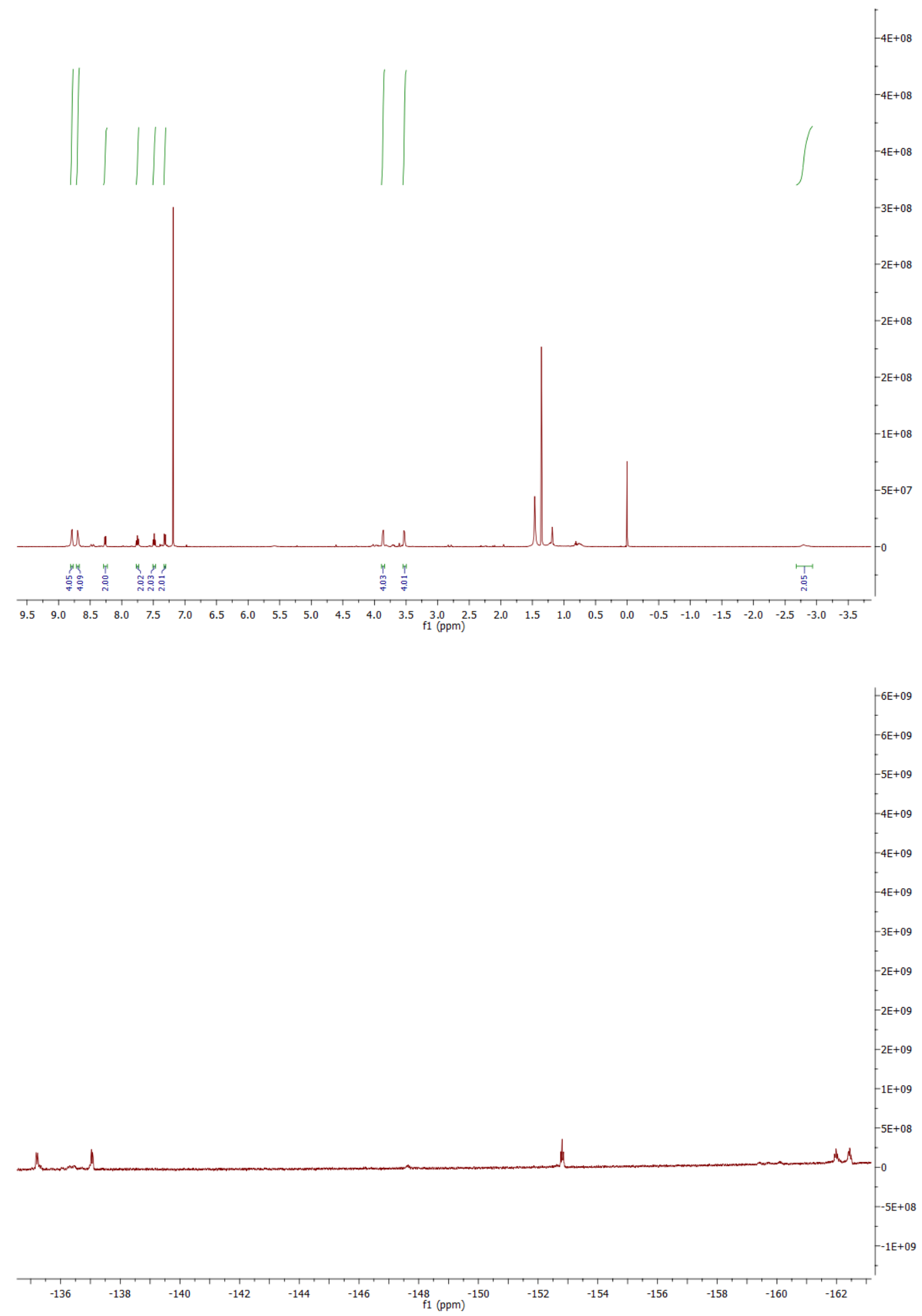
VIII.4 $2^{10}, 2^{20}$-bis(phenyl)-4,7,12,15-tetraoxa-2(5,15)-porpyhrina-1,3(1,2)dibenzena-cyclopentadecaphane-9-yn-8,11-dione (15b)

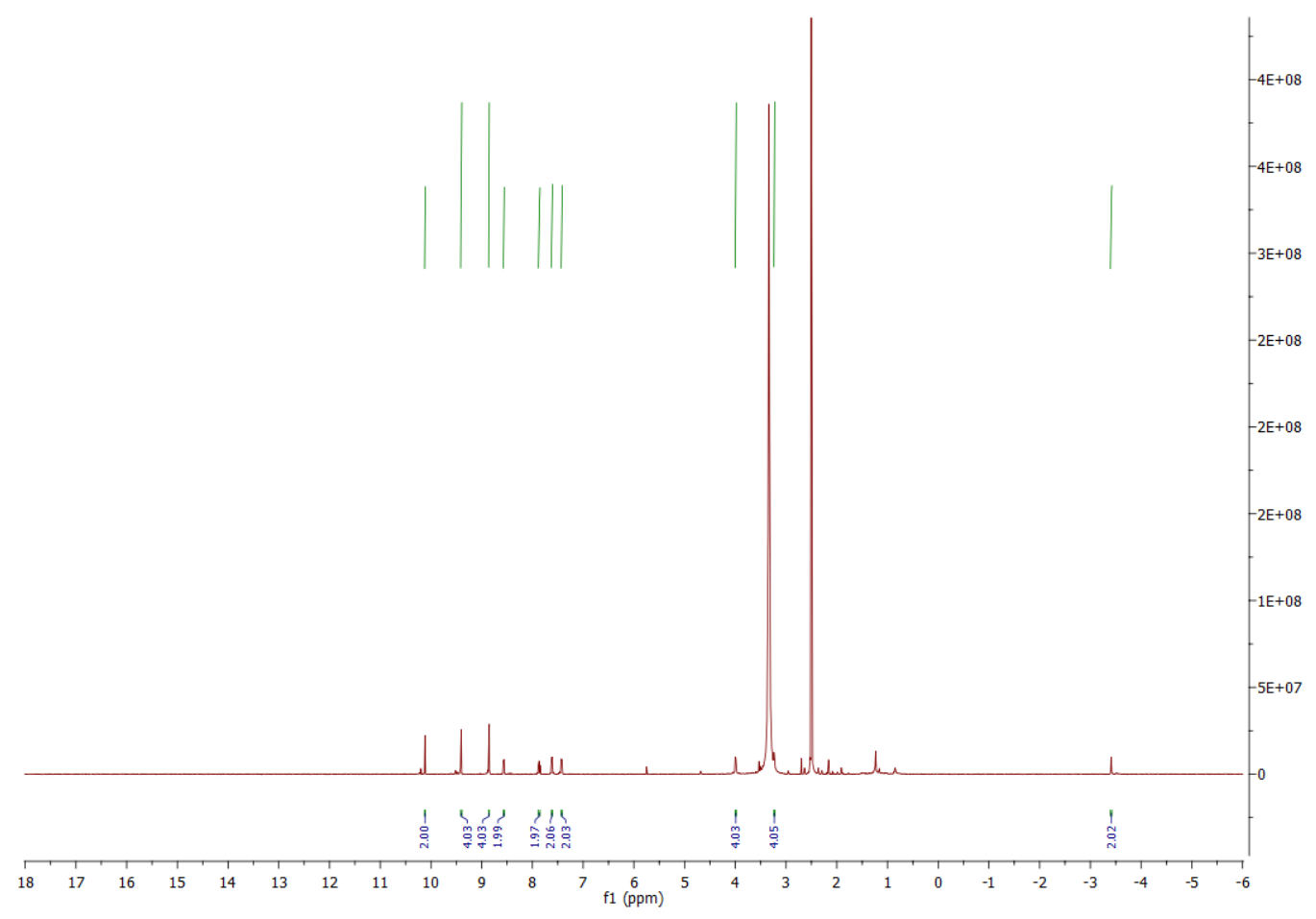

VIII.5 4,7,12,15-tetraoxa-2(5,15)-porpyhrina-1,3(1,2)-dibenzenacyclopentadecaphane-9-yn-8,11-dione (15a)

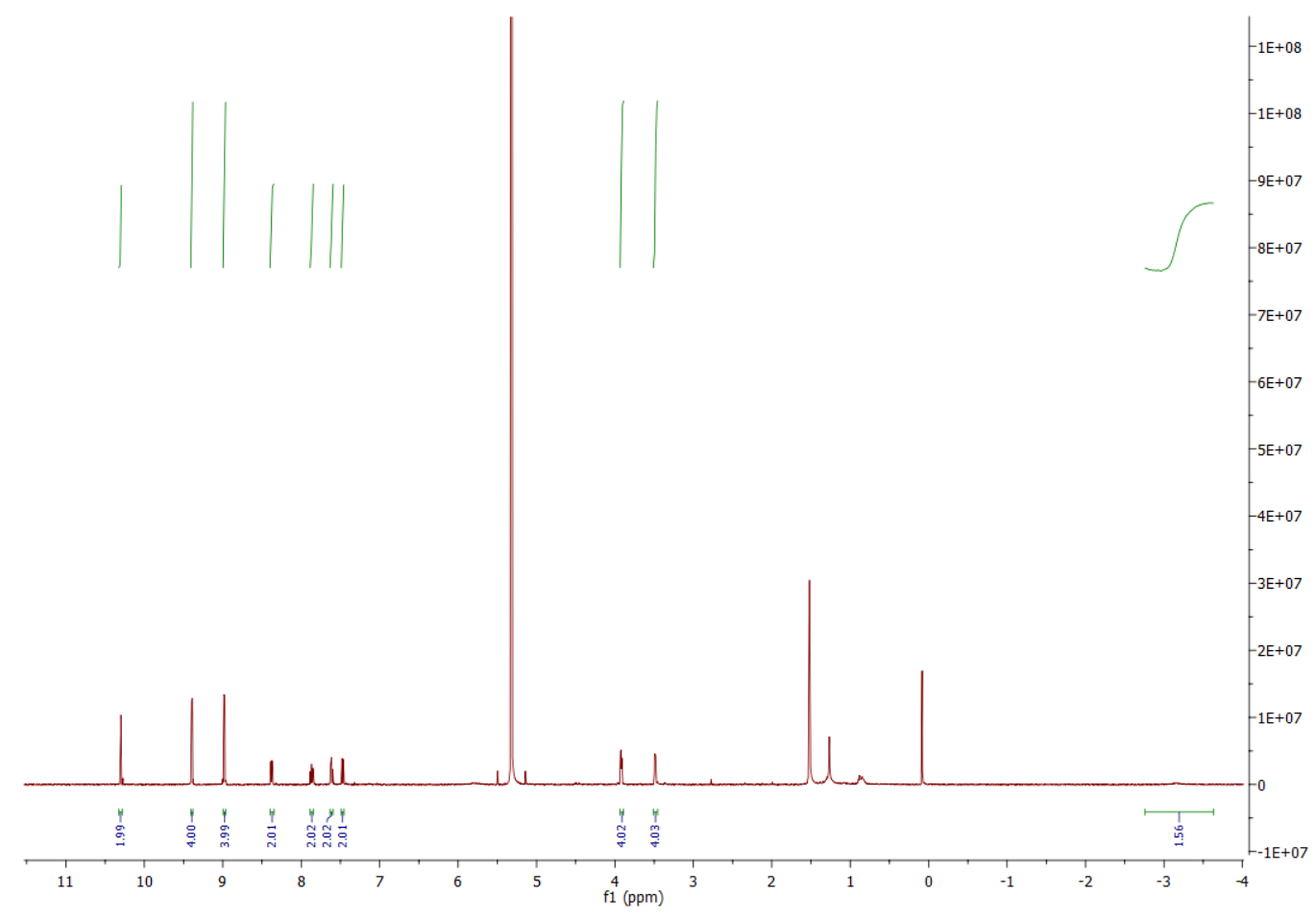


VIII.6 $2^{10}, 2^{20}$-bis(2,6-dichlorophenyl)-4,7,12,15-tetraoxa-2(5,15)-porpyhrina1,3(1,2)-dibenzena-cycloheptadecaphane-9-yn-8,11-dione (15c)

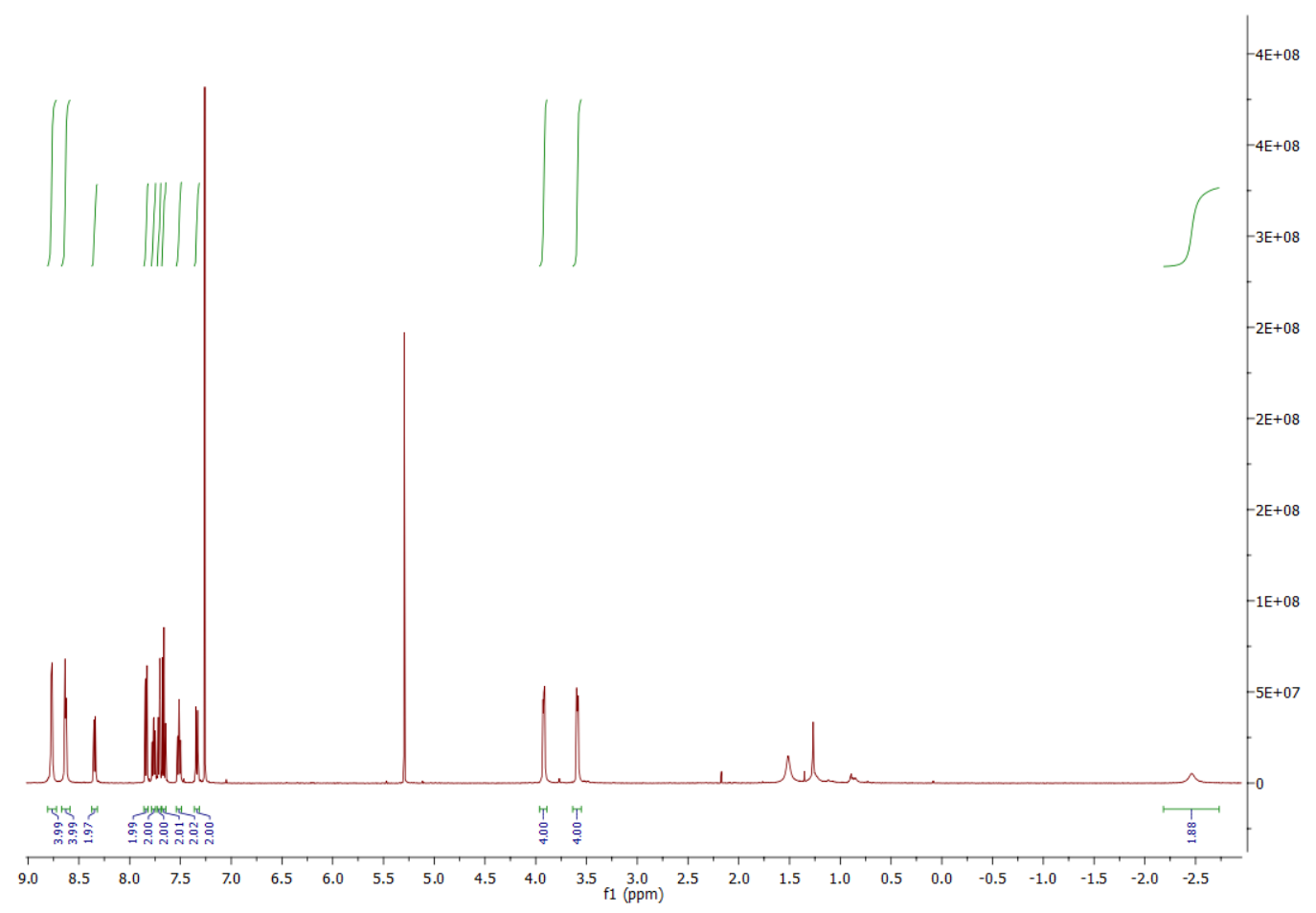

VIII.7 $2^{10}, 2^{20}$-bis(2,3,4,5,6-pentafluorophenyl)-4,8,13,17-tetraoxa-2(5,15)porpyhrina-1,3(1,2)-dibenzena-cycloheptadecaphane-10-yn-9,12-dione (15f)

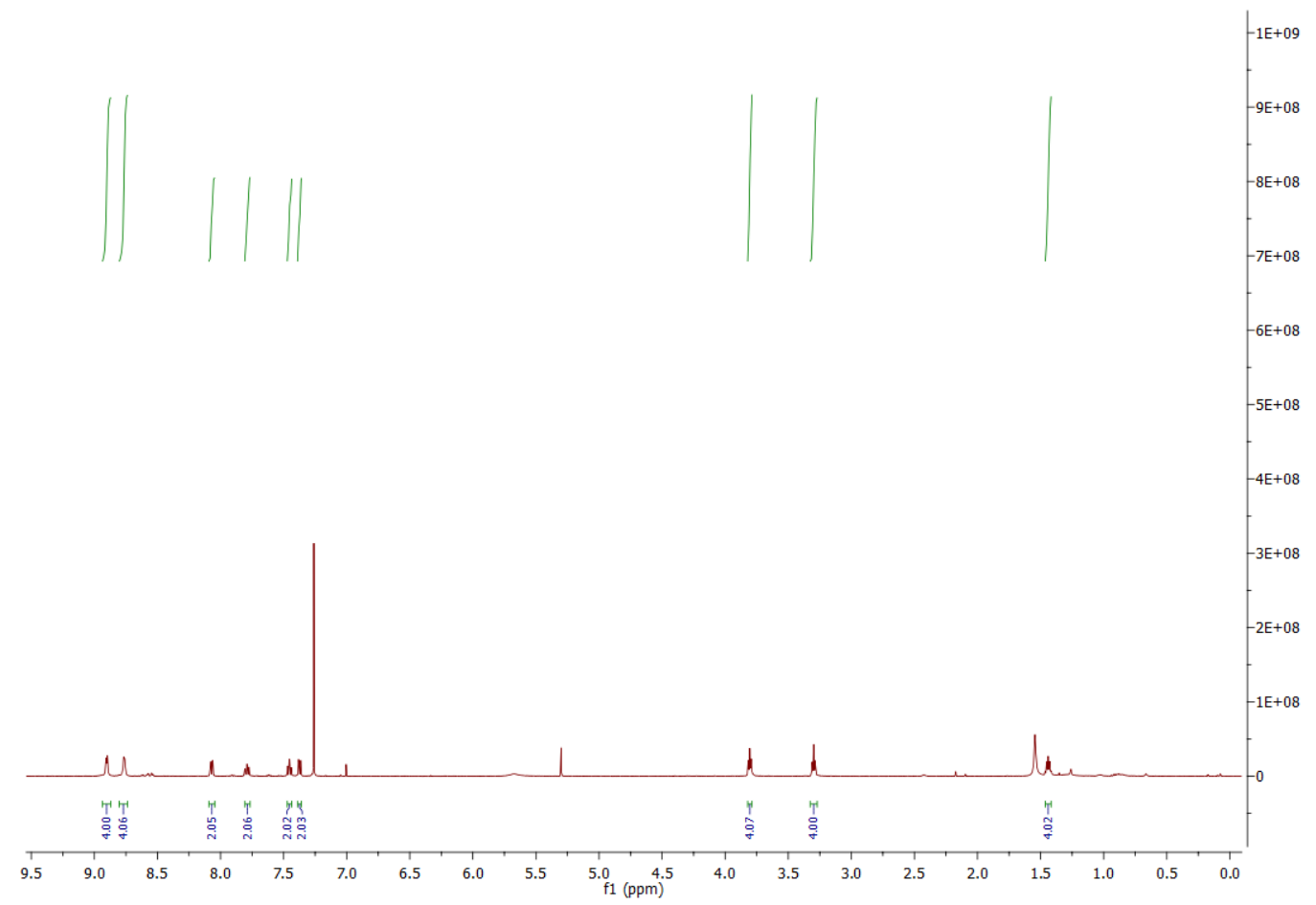




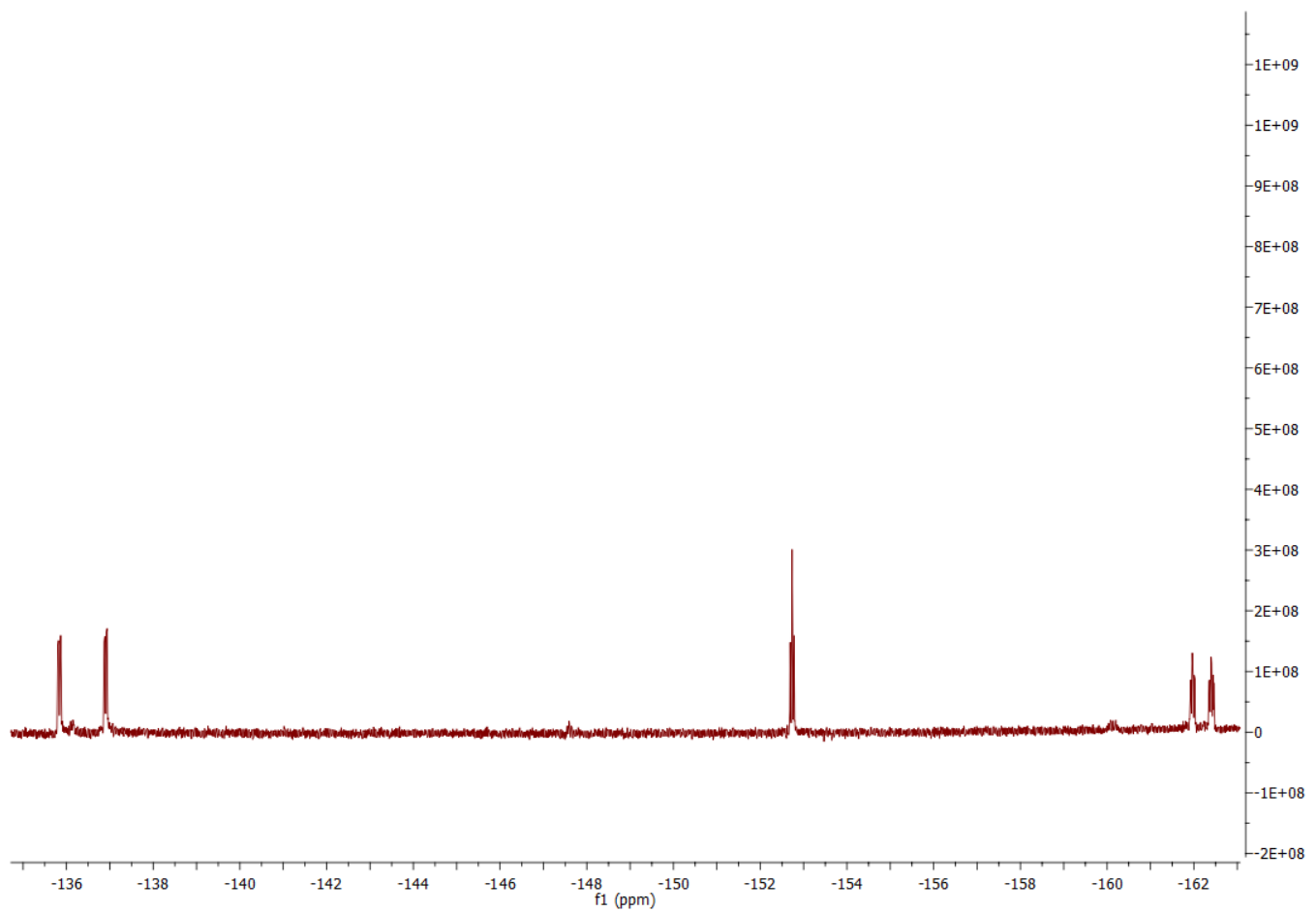

VIII.8 $2^{10}, 2^{20}$-bis(2,6-dichlorophenyl)-4,8,13,17-tetraoxa-2(5,15)-porpyhrina1,3(1,2)-dibenzena-cyclopentadecaphane-10-yn-9,12-dione (15e)

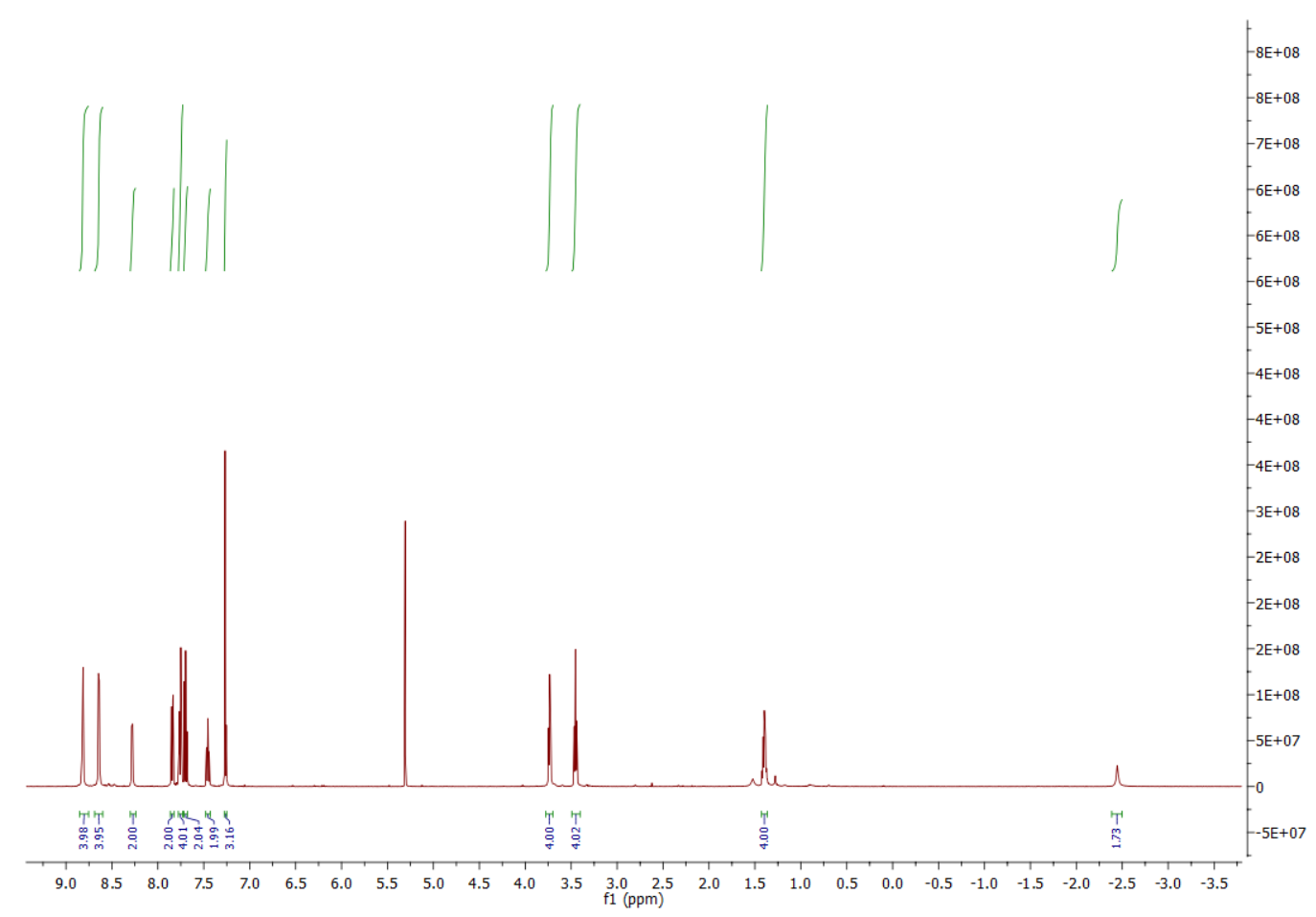


VIII.9 $2^{10}, 2^{20}$-bis(2,3,4,5,6-pentafluorophenyl)-4,7,11,14-tetraoxa-2(5,15)porpyhrina-9(4,5)-1,2,3-triazola-1,3(1,2)-dibenzena-cyclotetradecaphane-8,10dione (16d)

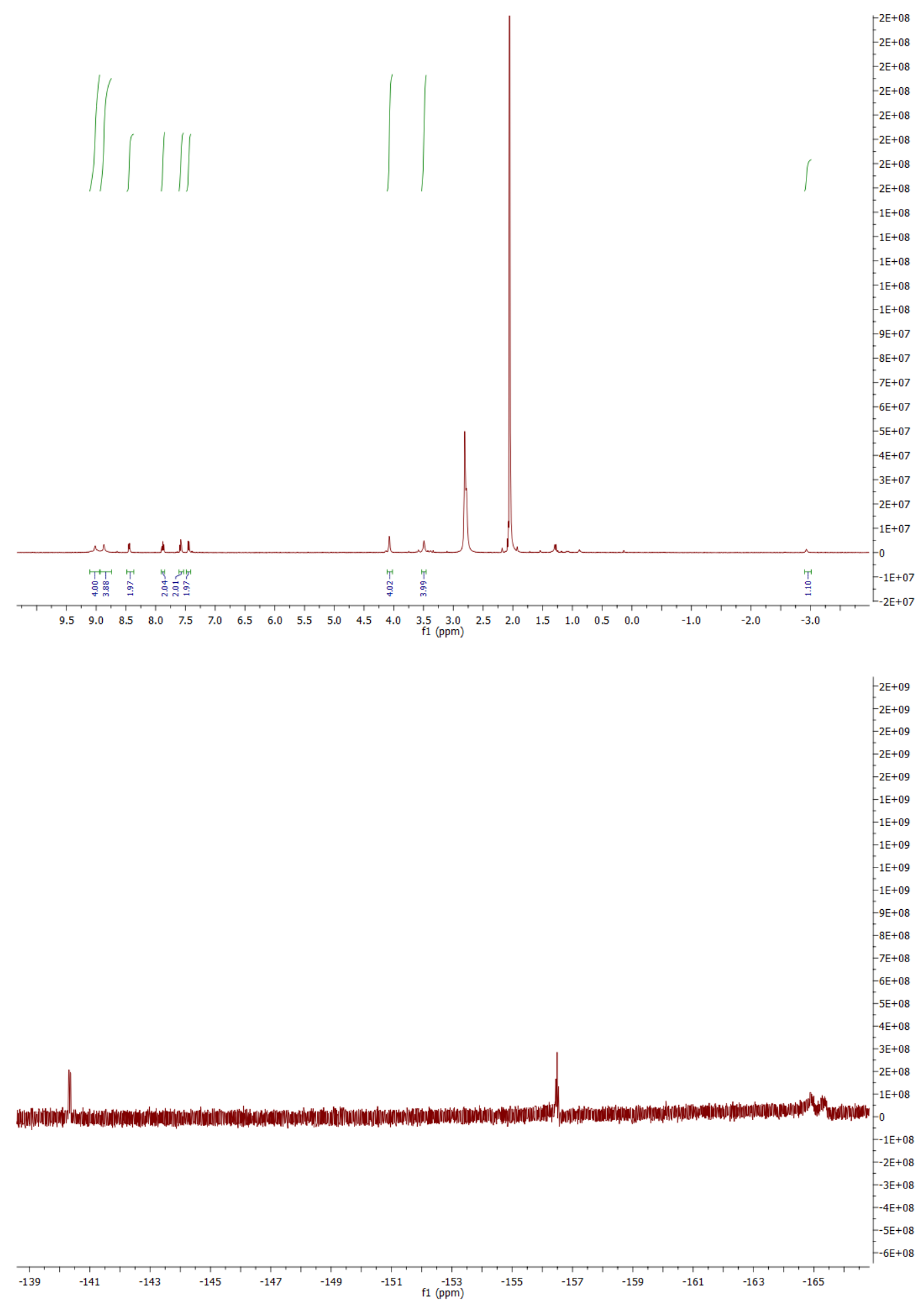


VIII.10 $\quad 2^{10}, 2^{20}$-bis(2,6-dichlorophenyl)-4,7,11,14-tetraoxa-2(5,15)-porpyhrina9(4,5)-1,2,3-triazola-1,3(1,2)-dibenzena-cyclotetradecaphane-8,10-dione (16c)

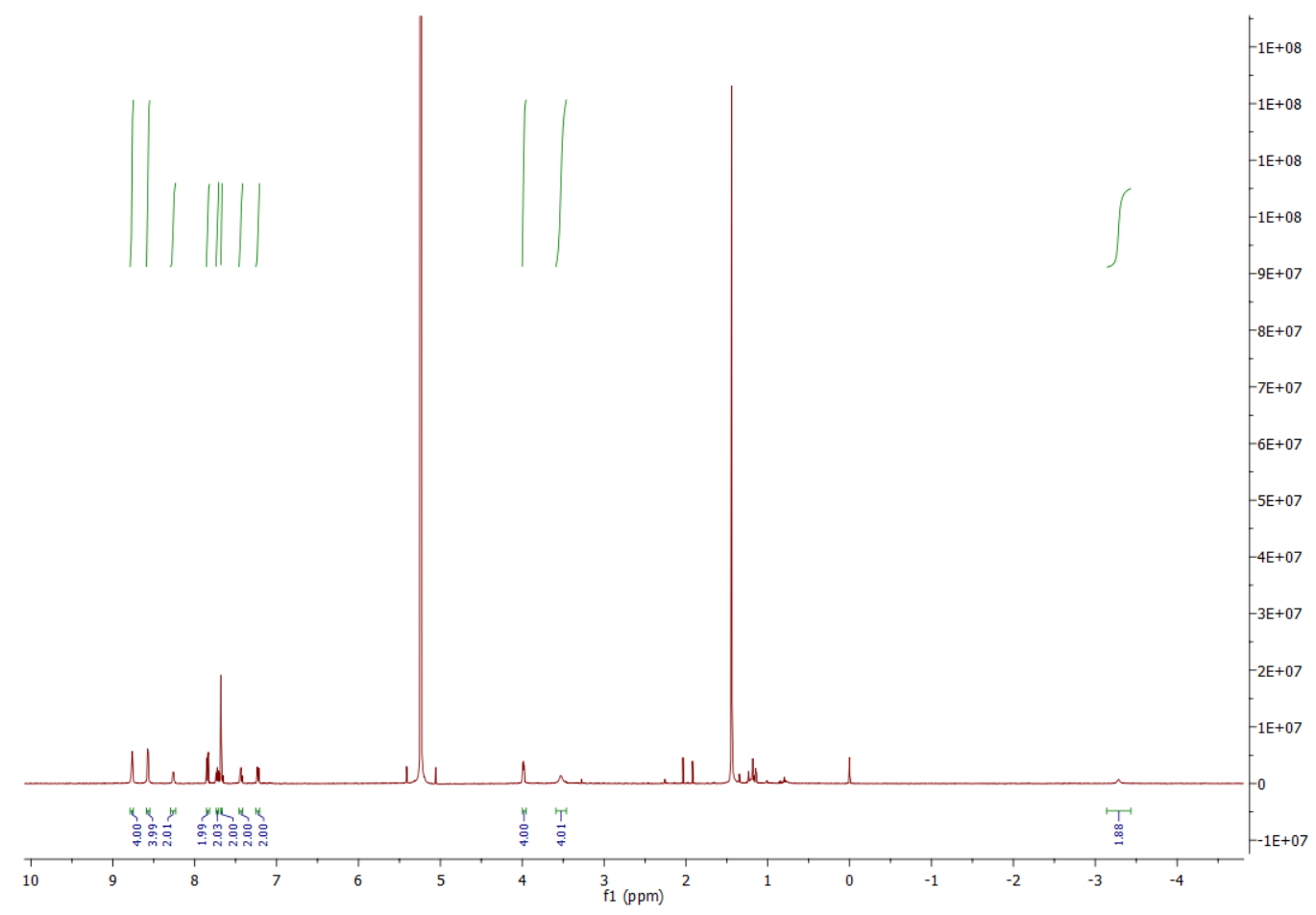

VIII.11 $2^{10}, 2^{20}$-bis(2,3,4,5,6-pentafluorophenyl)-4,8,12,16-tetraoxa-2(5,15)porpyhrina-10(4,5)-1,2,3-triazola-1,3(1,2)-dibenzena-cyclohexadecaphane-9,11dione (16f)

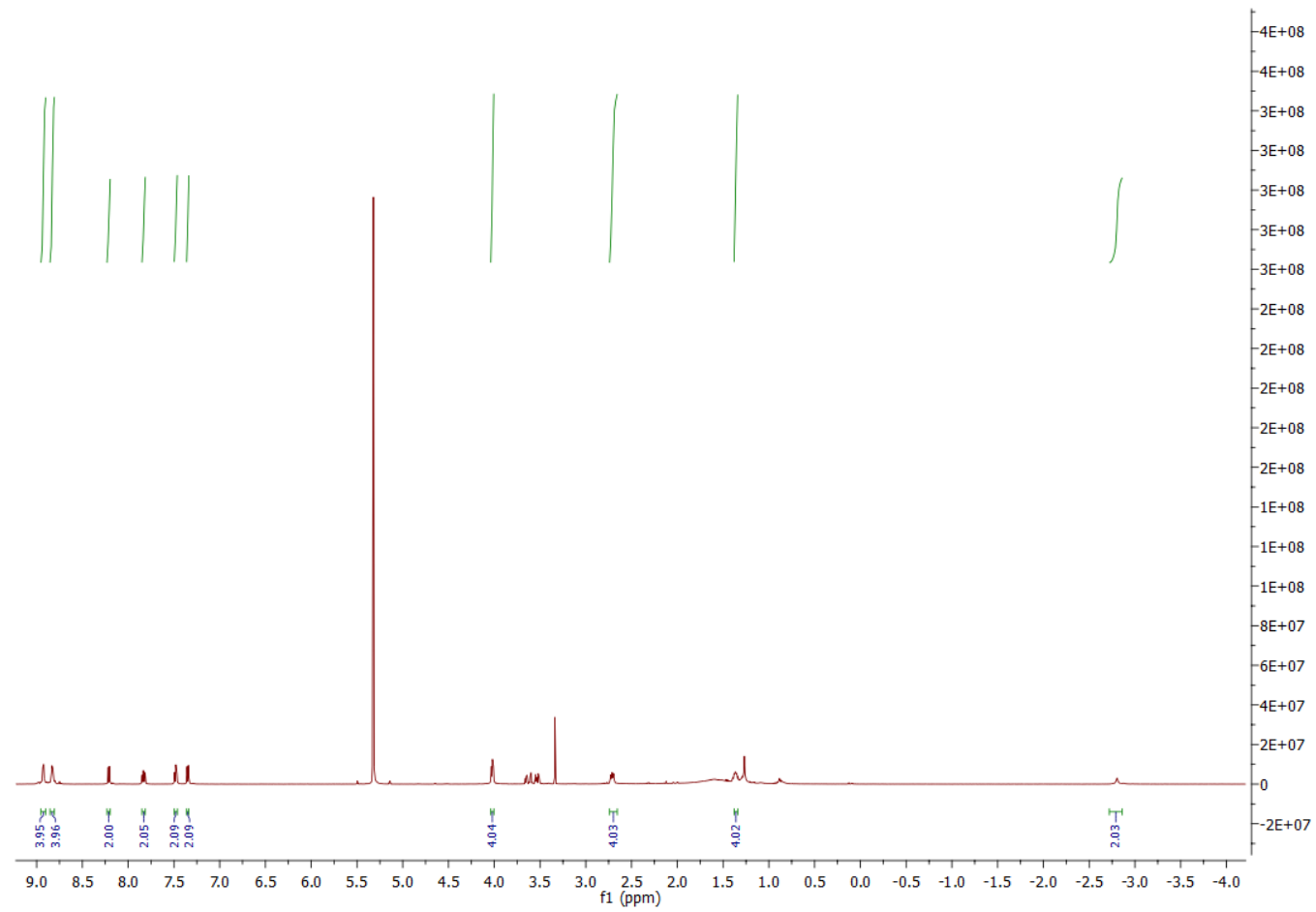




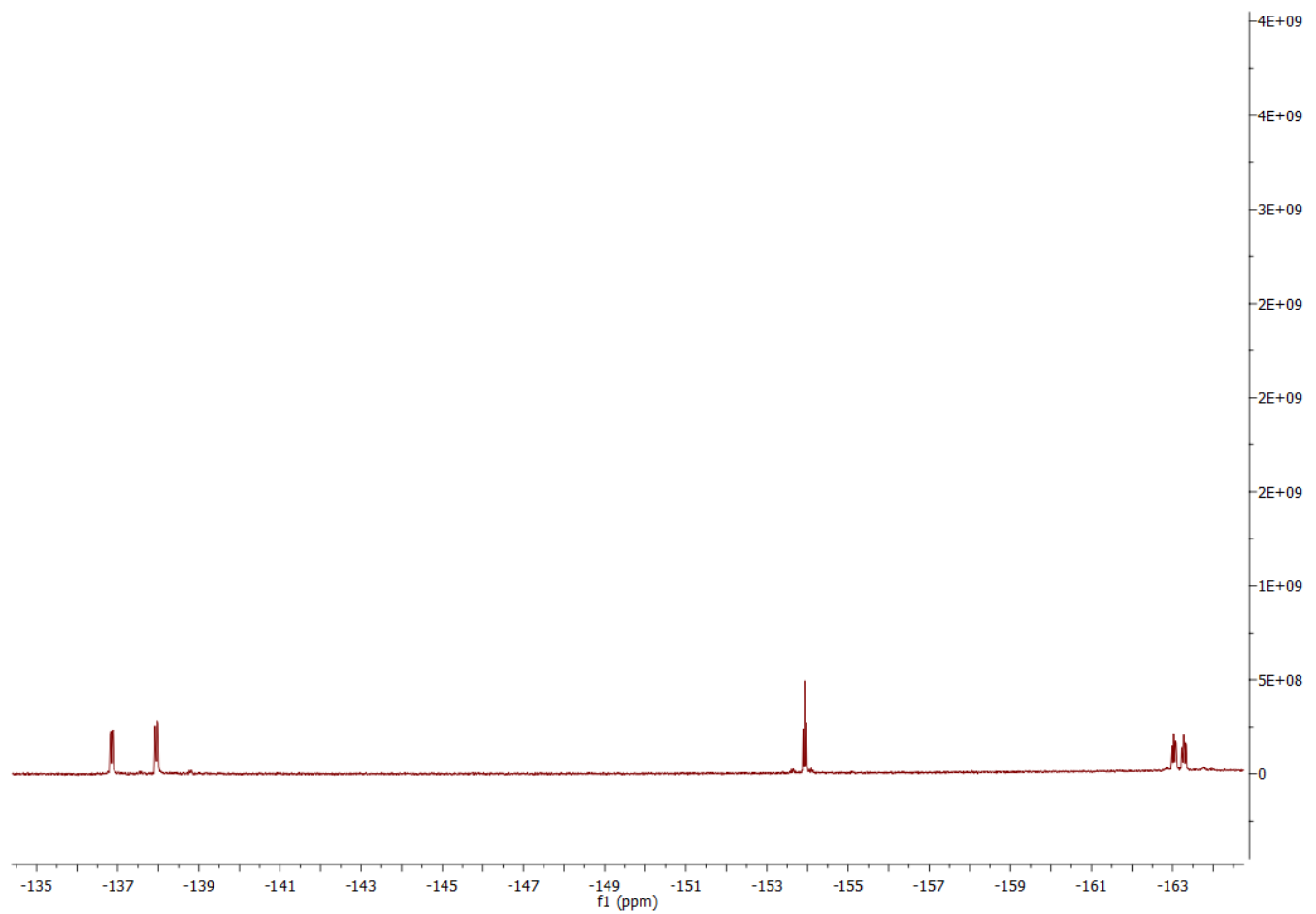

VIII.12 $2^{10}, 2^{20}$-bis(2,6-dichlorophenyl)-4,8,12,16-tetraoxa-2(5,15)-porpyhrina10(4,5)-1,2,3-triazola-1,3(1,2)-dibenzena-cyclohexadecaphane-9,11-dione (16e)

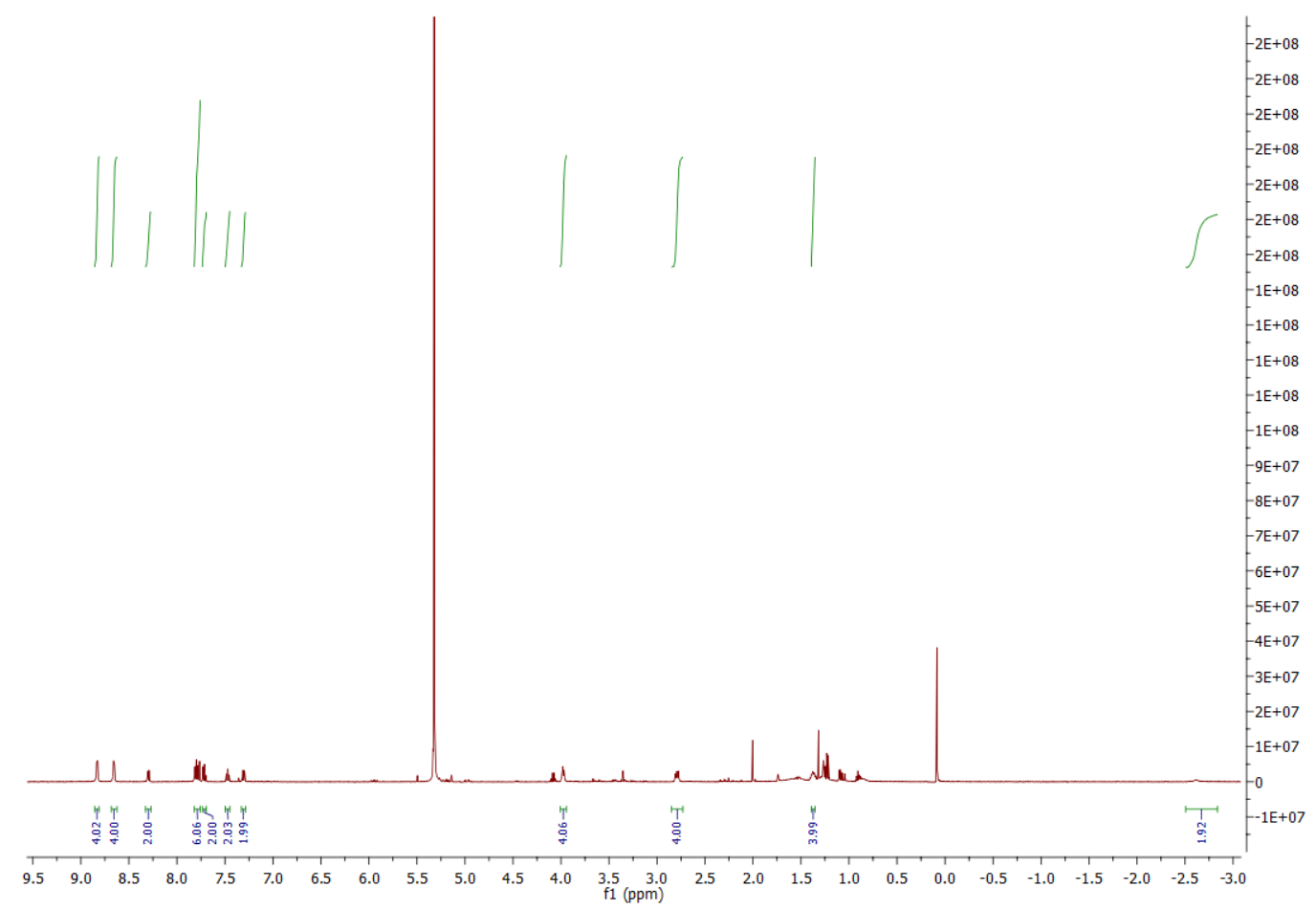


VIII.13 $2^{10}, 2^{20}$-bis(2,3,4,5,6-pentafluorophenyl)-4,7,11,14-tetraoxa-2(5,15)iron(III)porpyhrina-9(4,5)-1,2,3-triazola-1,3(1,2)-dibenzena-cyclotetradecaphane8,10-dione chloride (17e)

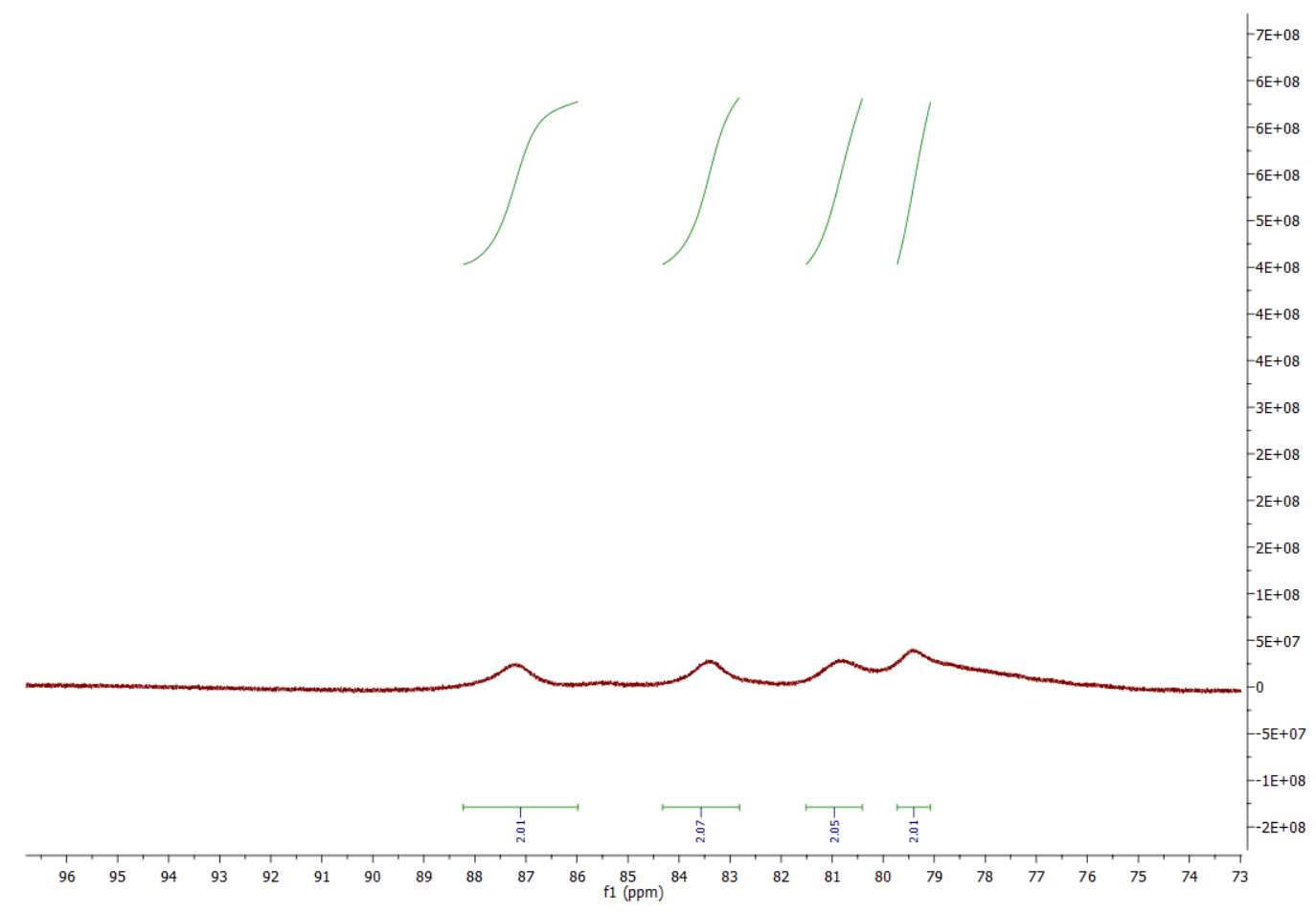

VIII.14 $2^{10}, 2^{20}$-bis(phenyl)-4,7,11,14-tetraoxa-2(5,15)-iron(III)porpyhrina-9(4,5)1,2,3-triazola-1,3(1,2)-dibenzena-cyclotetradecaphane-8,10-dione chloride (17b)

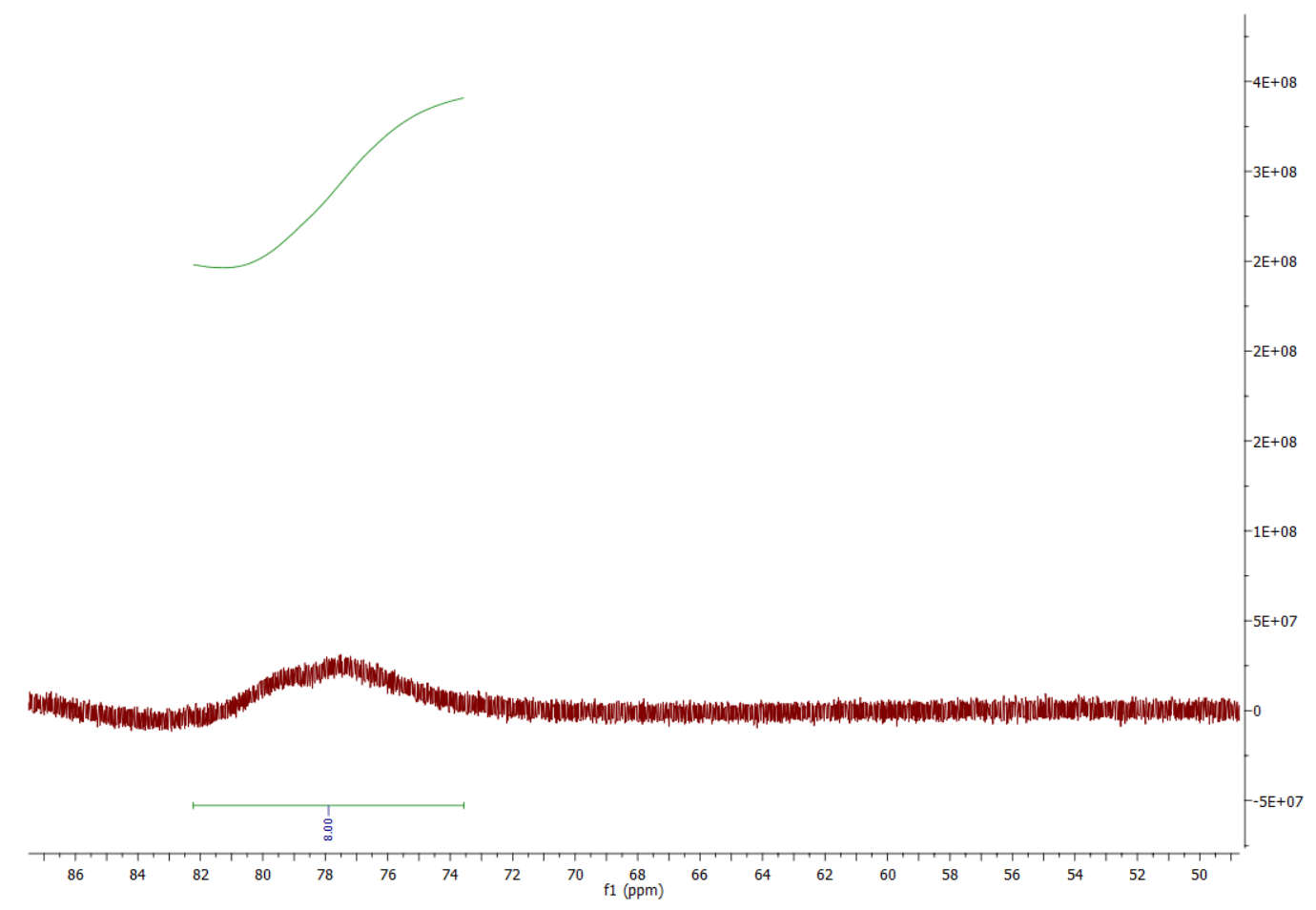


VIII.15 Synthesis of 4,7,11,14-tetraoxa-2(5,15)-iron(III)porpyhrina-9(4,5)-1,2,3triazola-1,3(1,2)-dibenzena-cyclotetradecaphane-8,10-dione chloride (17a)

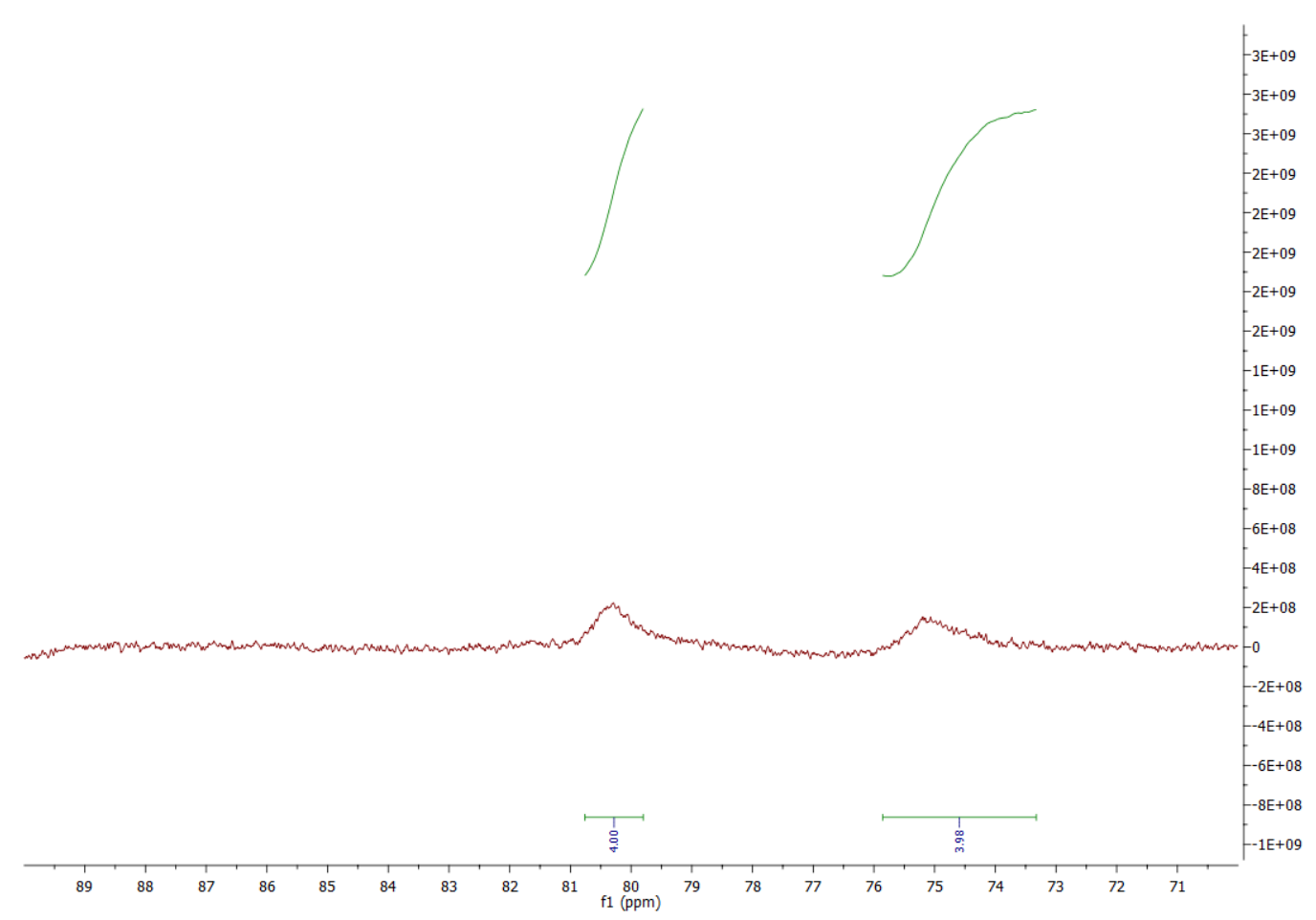

VIII.16 $2^{10}, 2^{20}$-bis(2,6-dichlorophenyl)-4,7,11,14-tetraoxa-2(5,15)-

iron(III)porpyhrina-9(4,5)-1,2,3-triazola-1,3(1,2)-dibenzena-cyclotetradecaphane8,10-dione chloride (17c)

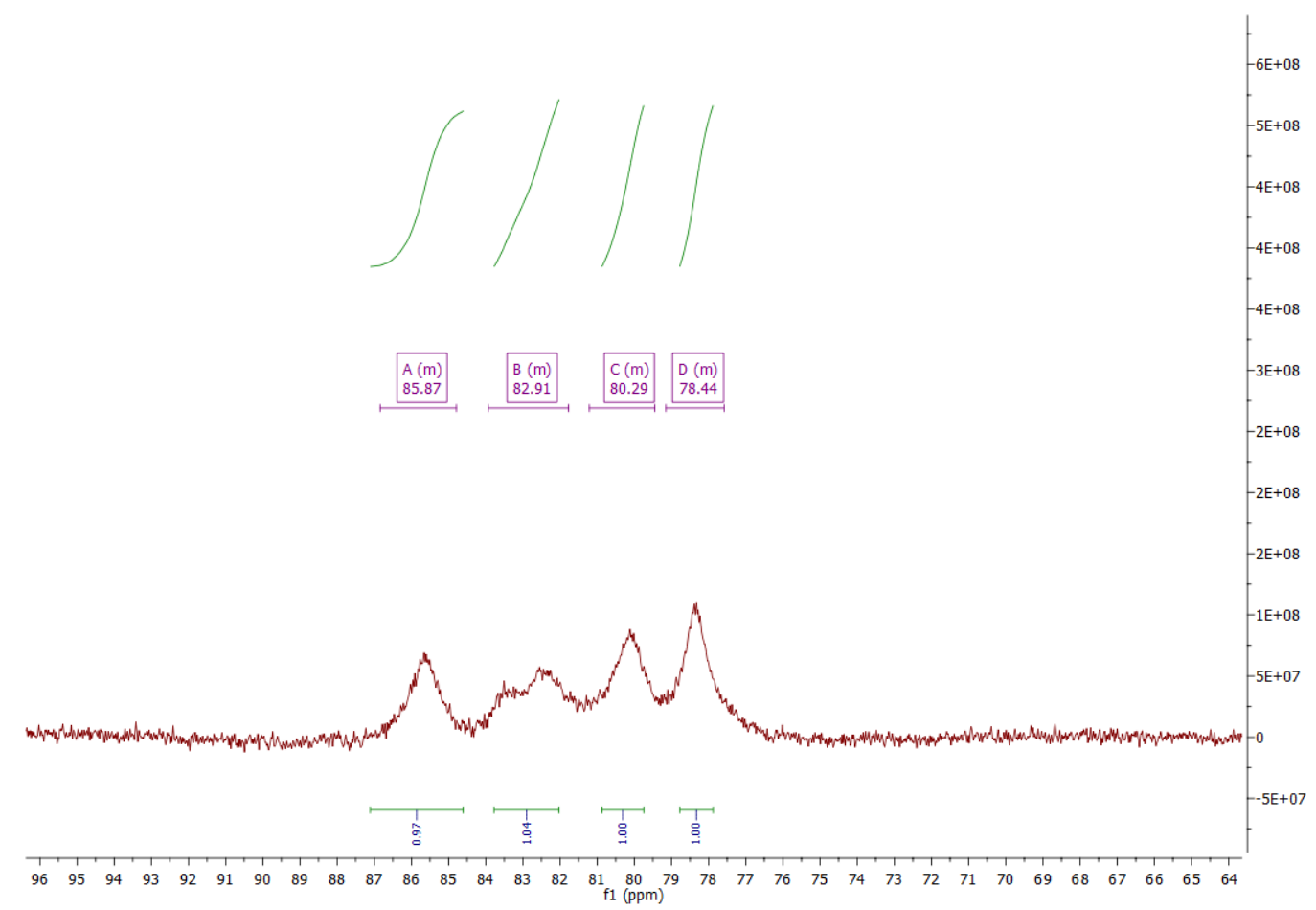


VIII.17 $2^{10}, 2^{20}$-bis(2,6-dichlorophenyl)-4,8,12,16-tetraoxa-2(5,15)-

iron(III)porpyhrina-10(4,5)-1,2,3-triazola-1,3(1,2)-dibenzena-

cyclohexadecaphane-9,11-dione chloride (17d)

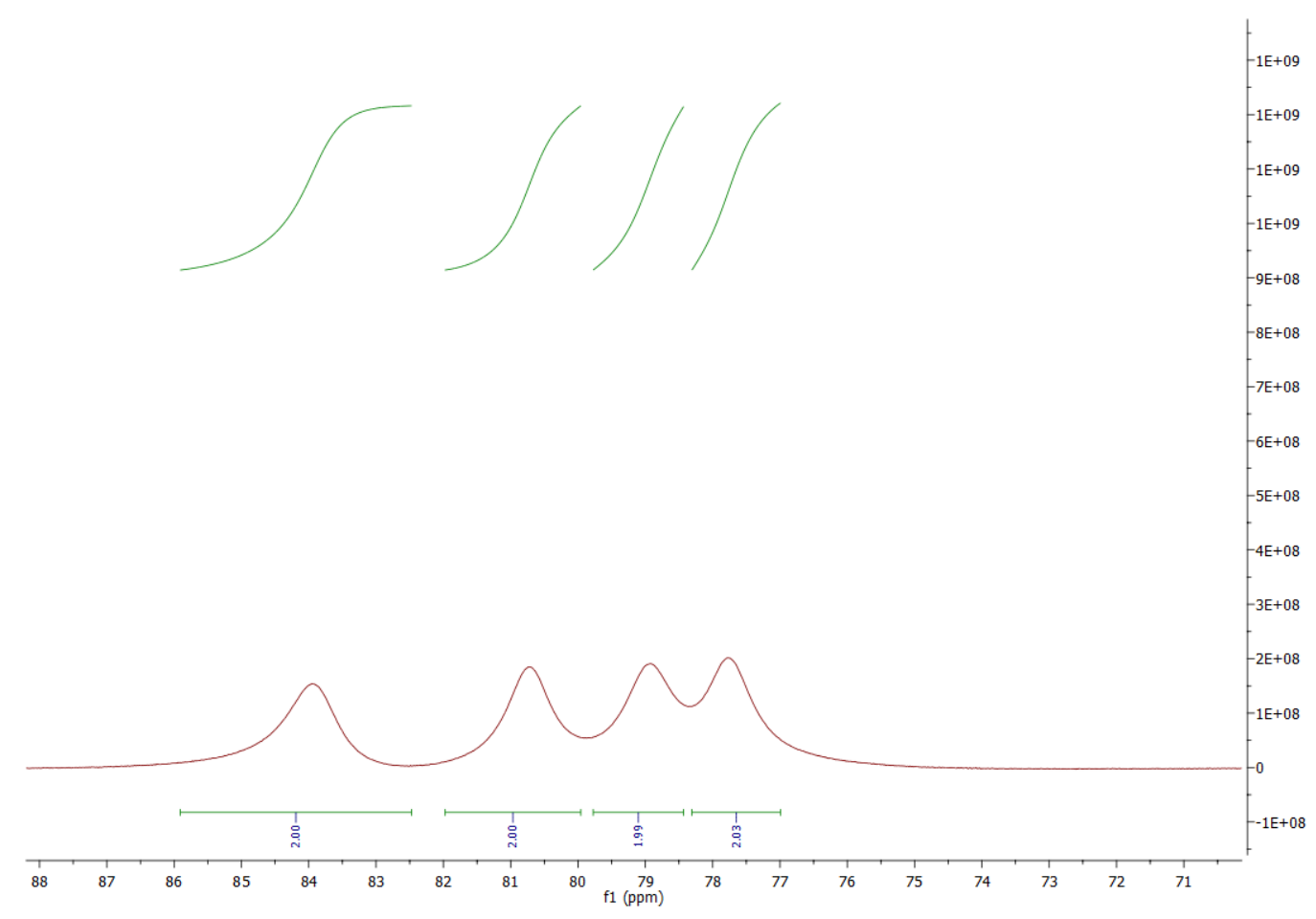


VIII.18 $2^{10}, 2^{20}$-bis(2,6-dichlorophenyl)-4,7,11,14-tetraoxa-2(5,15)-

zinc(II)porpyhrina-9(4,5)-1,2,3-triazola-1,3(1,2)-dibenzena-cyclotetradecaphane8,10-dione

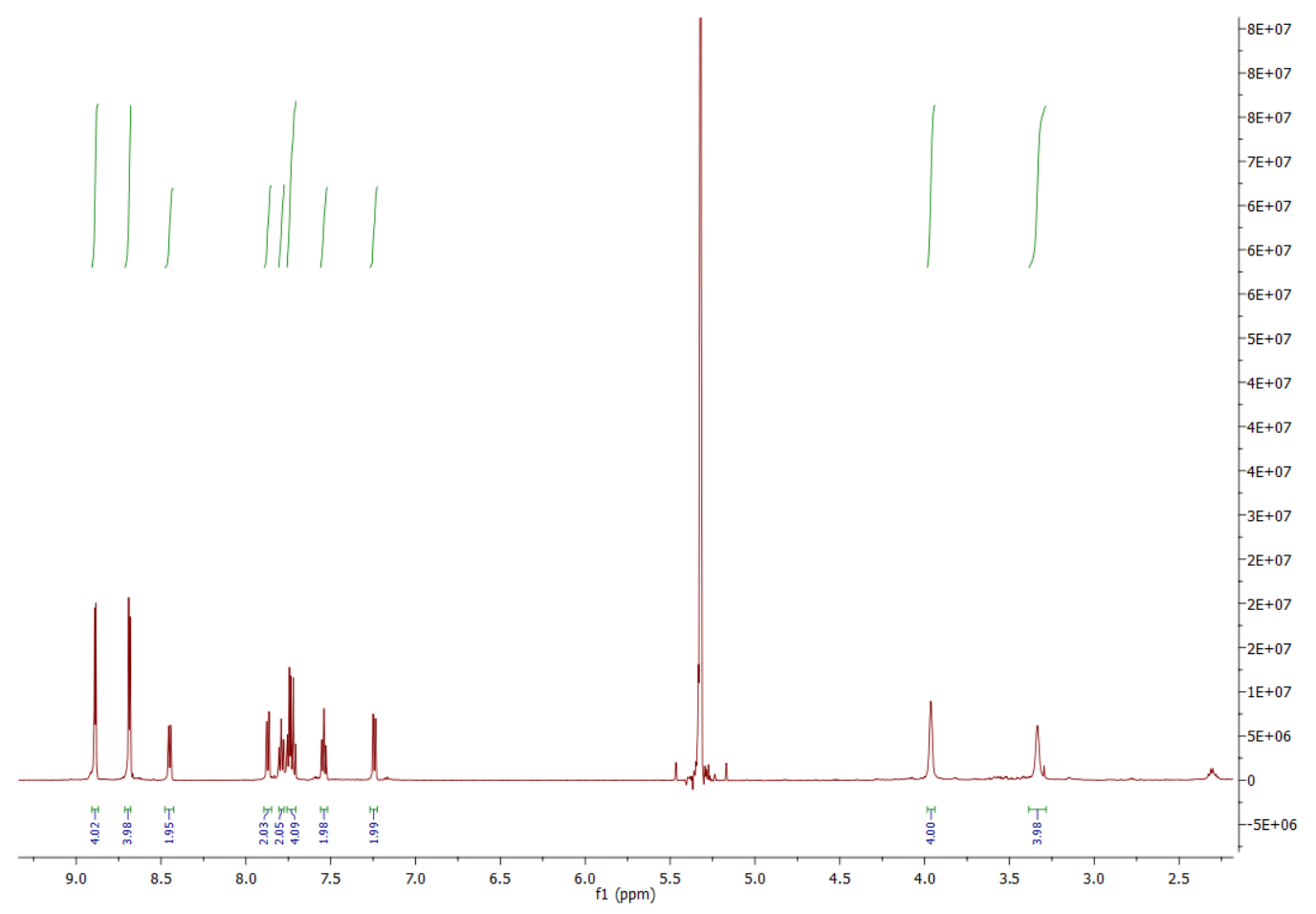


VIII.19 $2^{10}, 2^{20}$-bis(2,6-dichlorophenyl)-4,8,12,16-tetraoxa-2(5,15)-

zinc(II)porpyhrina-10(4,5)-1,2,3-triazola-1,3(1,2)-dibenzena-

cyclohexadecaphane-9,11-dione

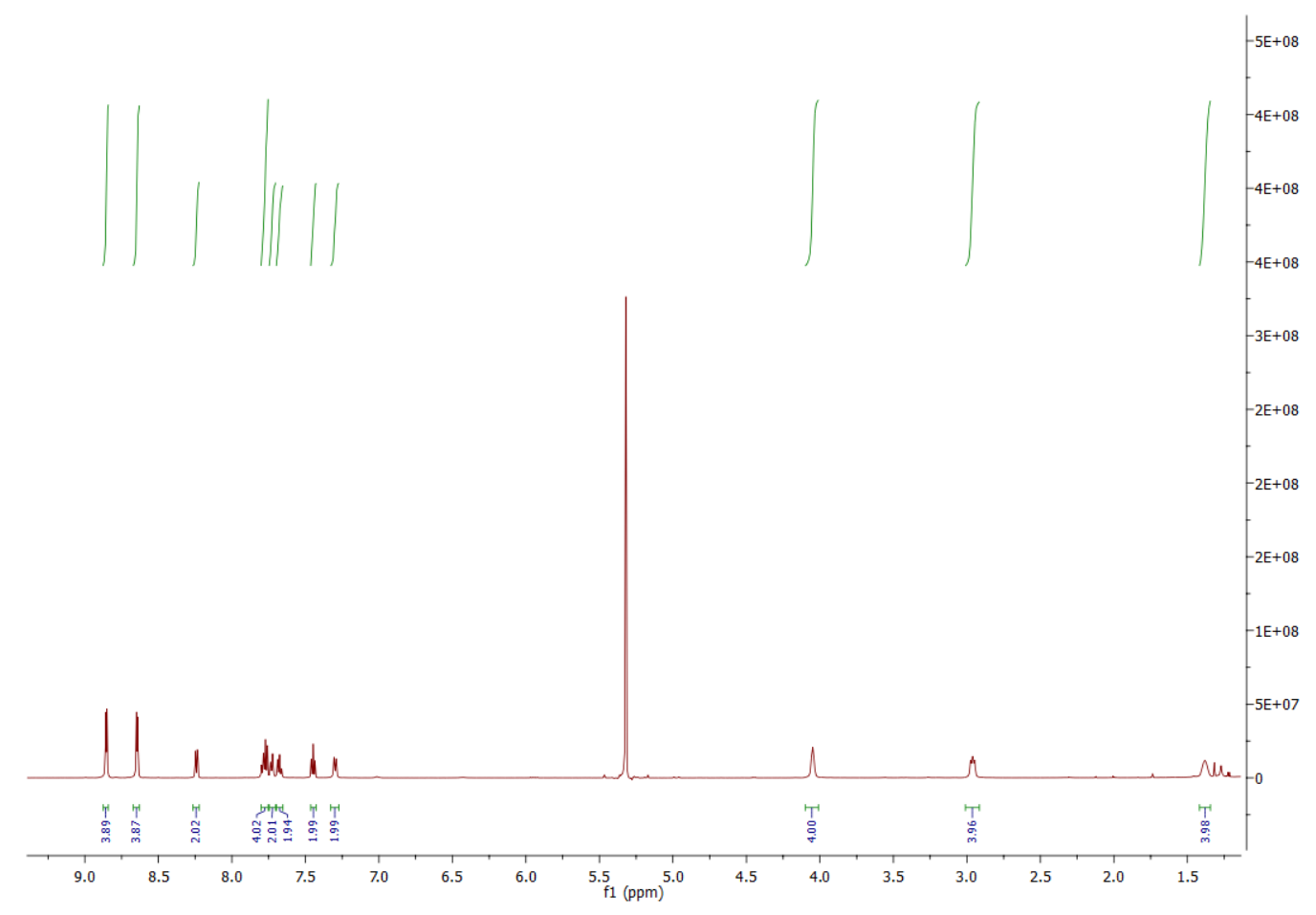




\section{References}

1. Littler, B. J.; Miller, M. A.; Hung. C.-H.; Wagner, R. W.; O'Shea, D. F.; Boyle, P. D.; Lindsey, J. A. Refined Synthesis of 5-Substituted Dipyrromethanes, J. Org. Chem. 1999, 64, 1391-1396.

2. He, J.; Zheng, J.; Liu, J.; She, X.; Pan, X. N-Heterocyclic Carbene Catalyzed Nucleophilic Substitution Reaction for Construction of Benzopyrones and Benzofuranones, Org. Lett., 2006, 8, 4637-4640.

3. Perdew, J. P.; Burke, K.; Ernzerhof, M. Generalized gradient approximation made simple, Phys. Rev. Lett.,1996, 77, 3865-3868.

4. Grimme, S.; Antony, J.; Ehrlich S.; Krieg, H. A consistent and accurate ab initio parameterization of density functional dispersion correction (DFT-D) for the 94 elements H-Pu, J. Chem. Phys., 2010, 132, 154104.

5. Turbomole7.2: TURBOMOLE V7.2 2017, a development of University of Karlsruhe and Forschungszentrum Karlsruhe $\mathrm{GmbH}$, 1989-2007, TURBOMOLE GmbH, since 2007; available from http://www.turbomole.com.

6. X-Area program package, STOE \& CIE, Darmstadt, Germany.

7. Sheldrick, G. M. A short history of SHELX, Acta Crystallogr., Sect. A 2008, 64, 112-122.

8. Sheldrick, G. M., SHELXT - Integrated space-group and crystal-structure determination, Acta Cryst., 2015, C71, 3-8.

9. Spek, A. L. PLATON SQUEEZE: a tool for the calculation of the disordered solvent contribution to the calculated structure factors, Acta Crystallogr., Sect. C: Struct. Chem. 2015, 71, 9.

10. Bolte, M., TWINLAW and HKLF5: two programs for the handling of nonmerohedral twins, J. Appl. Cryst., 2004, 37, 162-165.

11. CrysAlisPro 2015, v 1.171.38.43; Rigaku Oxford Diffraction.

12. Dolomanov, O. V.; Bourhis, L. J.; Gildea, R. J.; Howard, J. A. K.;Puschmann,H. OLEX2: a complete structure solution, refinement and analysis program, J. Appl. Crystallogr. 2009, 42, 339-341. 